\title{
Sensor Fusion Based Fault-Tolerant Attitude Estimation Solutions for Small Unmanned Aerial Vehicles
}

Jason Nicholas Gross

West Virginia University

Follow this and additional works at: https://researchrepository.wvu.edu/etd

\section{Recommended Citation}

Gross, Jason Nicholas, "Sensor Fusion Based Fault-Tolerant Attitude Estimation Solutions for Small Unmanned Aerial Vehicles" (2011). Graduate Theses, Dissertations, and Problem Reports. 3071.

https://researchrepository.wvu.edu/etd/3071

This Dissertation is protected by copyright and/or related rights. It has been brought to you by the The Research Repository @ WVU with permission from the rights-holder(s). You are free to use this Dissertation in any way that is permitted by the copyright and related rights legislation that applies to your use. For other uses you must obtain permission from the rights-holder(s) directly, unless additional rights are indicated by a Creative Commons license in the record and/ or on the work itself. This Dissertation has been accepted for inclusion in WVU Graduate Theses, Dissertations, and Problem Reports collection by an authorized administrator of The Research Repository @ WVU.

For more information, please contact researchrepository@mail.wvu.edu. 


\title{
Sensor Fusion Based Fault-Tolerant Attitude Estimation Solutions for Small Unmanned Aerial Vehicles
}

\author{
Jason Nicholas Gross
}

\author{
Dissertation submitted to the \\ College of Engineering and Mineral Resources \\ at West Virginia University \\ in partial fulfillment of the requirements \\ for the degree of \\ Doctor of Philosophy \\ in \\ Aerospace Engineering
}

Approved By:

Dr. Marcello Napolitano, Chair, Research Advisor

Dr. Yu Gu, Research Co-Advisor

Dr. Larry Banta

Dr. Wade Huebsch

Dr. Powsiri Klinkhachorn

Department of Mechanical and Aerospace Engineering

Morgantown, West Virginia

2011

Keywords: Sensor Fusion; Attitude Estimation; Kalman Filtering;

Unmanned Aerial Vehicle; Fault-Tolerance 


\section{Abstract}

\section{Sensor Fusion Based Fault-Tolerant Attitude Estimation Solutions for Small Unmanned Aerial Vehicles \\ by Jason Nicholas Gross}

Navigation-grade inertial sensors are often too expensive and too heavy for use in most Small Unmanned Aerial Vehicle (SUAV) systems. Low-cost Micro-Electrical-Mechanical-Systems (MEMS) inertial sensors provide an attractive alternative, but currently do not provide an adequate navigation solution alone due to the presence of sensor bias. Toward addressing this problem, this research focuses on the development and experimental evaluation of sensor fusion algorithms to combine partially redundant information from low-cost sensor to achieve accurate SUAV attitude estimation. To conduct this research, several sets of SUAVs flight data that include measurements from a low-cost MEMS based Inertial Measurement Unit, a Global Positioning System receiver, and a set of low-grade tri-axial magnetometers are used to evaluate a variety of algorithms. In order to provide a baseline for performance evaluation, attitude measurements obtained directly with a high-quality mechanical vertical gyroscope are used as an independent attitude 'truth'. In addition, as a part of this project, a custom SUAV avionics system was developed to provide a platform for fault-tolerant flight control research.

The overall goal of this research is to provide high-accuracy attitude estimation during nominal sensor performance conditions and in the event of sensors failures, while using only low-cost components. To achieve this goal, this study is carried out in three phases. The specific aim of the first phase is to obtain high-accuracy under nominal sensor conditions. During this phase, two different nonlinear Kalman filtering methods are applied to various sensor fusion formulations and evaluated with respect to estimation accuracy over diverse sets of flight data. Next, during the second phase, sensor fusion based calibration techniques are explored to further enhance estimation accuracy. Finally, the third phase of the study considers the design of a sensor fusion attitude estimation architecture that rejects sensor failures and supports a graceful loss of performance in the event of sensor failures. This algorithm is based on a nonlinear information filter and features a novel Failure Detection, Identification, and Accommodation (FDIA) approach. During this phase, it is shown that the sensor fusion algorithm handles both abruptly occurring large magnitude sensor failures, as well as gradually growing small magnitude sensor failures. 


\section{Acknowledgements}

First and foremost, I would like to thank my graduate research co-advisors Dr. Marcello Napolitano and Dr. Yu Gu. Without your support and guidance, this project would not have been possible.

Dr. Napolitano, your support and mentorship made graduate school a great educational experience. I am grateful for the opportunity to work on many interesting problems as a part of your research group. Thank you.

Dr. Gu, your constant advice, encouragement, and thought provoking insights have defined the success of this dissertation project. I really appreciate the amount of time you dedicated to mentoring me and offering contributions to this project. In particular, your theory of three levels in terms of "robustness, adaptation, and switching" became the theme of this project in the form of "sensor fusion, on-line calibration, and the information filter." Thank you.

I would also like to thank my committee members Dr. Larry Banta, Dr. Wade Huebsch, and Dr. Powsiri Klinkachorn. Thank you for providing helpful feedback.

Thank you Dr. Srikanth Gururajan, your advice and support have been very helpful, and I appreciate your commitment to our flight testing activities. I would also like to thank Dr. Brad Seanor. I have appreciated your help and encouragement.

I would like to thank my fellow graduate students in the research group Frank Barchesky, Marc Gramlich, Jason Jarrell, Zach Mercurio, Kerri Phillips, Matthew Rhudy, Daniele Tancredi and Sergio Tomayo.

In particular, I would like to thank and acknowledge the contribution of fellow $\mathrm{PhD}$ student Matthew Rhudy. Matt, the many fruitful discussions I had with you and Dr. Gu greatly influenced this work; especially our discussion of the covariance ellipsoids, in which you suggested defining a 'force-field'.

I would also like to thank Frank Barchesky for taking on many of the responsibilities associated with avionics hardware design upon joining the group. Frank, I admire you enthusiasm and ability to continually improve upon the design.

Finally, I would like to thank my soon to be wife Beth, my parents, and my brother Ryan. Thank you for your love and support. 


\section{Table of Contents}

$\begin{array}{lll}\text { CHAPTER } 1 \text { : INTRODUCTION } & 1\end{array}$

$\begin{array}{llr}1.1 & \text { Problem Description } & 1\end{array}$

$\begin{array}{llr}1.2 & \text { Organization } & 5\end{array}$

$\begin{array}{lll}\text { CHAPTER } 2 & \text { : LITERATURE REVIEW }\end{array}$

$\begin{array}{lll}2.1 & \text { Low-Cost Navigation Sensors } & 7\end{array}$

$\begin{array}{lll}2.1 .1 & \text { Inertial Navigation Systems } & 7\end{array}$

2.1.2 Global Positioning System 9

$\begin{array}{lll}2.1 .3 & \text { Magnetometers } & 10\end{array}$

2.2 State and Parameter Estimation $\quad 11$

2.2.1 Linear Optimal Kalman Filter $\quad 11$

2.2.2 Extended Kalman Filter $\quad 12$

$\begin{array}{lll}\text { 2.2.3 Sigma-Point Kalman Filter } & 13\end{array}$

$\begin{array}{ll}\text { 2.2.4 Information Filter } & 15\end{array}$

$\begin{array}{lll}2.3 & \text { GPS/INS Integrated Navigation Systems } & 16\end{array}$

$\begin{array}{lll}\text { 2.3.1 } & \text { GPS/INS Sensor Fusion System Classification } & 16\end{array}$

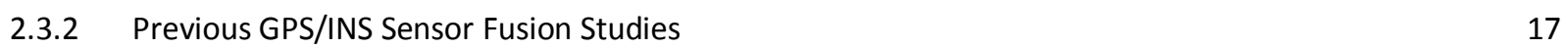

2.3.3 GPS/INS + Other Aiding Sensors based Integrated Navigation $\quad 19$

$\begin{array}{lll}\text { 2.3.4 Sensor Fusion Based Calibration } & 20\end{array}$

2.4 Sensor Fusion with Failure Detection and Accommodation 22

$\begin{array}{lll}2.5 & \text { Research SUAV Avionics System Design } & 23\end{array}$

$\begin{array}{lll}\text { CHAPTER } 3 & \text { : EXPERIMENTAL SET-UP } & 26\end{array}$

$\begin{array}{llr}\text { 3.1 WVU YF-22 Research UAV } & 26\end{array}$

\begin{tabular}{ll}
3.2 & Onboard Computer and Sensor System \\
\hline
\end{tabular}

$\begin{array}{lll}\text { 3.2.1 Design Requirements } & 27\end{array}$

3.2.2 YF-22 Formation Flight Avionics System $\quad 28$

$\begin{array}{lll}\text { 3.2.3 Gen-V Avionics System Development } & 30\end{array}$

$\begin{array}{lll}3.3 & \text { Sensor Fusion Truth Data } & 36\end{array}$

$\begin{array}{lll}\text { 3.4 Sensor Fusion Source Data Performance Specifications } & 36\end{array}$

$\begin{array}{llr}3.5 & \text { Flight Testing } & 38\end{array}$ 
$\begin{array}{lll}\text { CHAPTER } 5 & \text { : NONLINEAR ESTIMATION }\end{array}$

5.1 Discrete-Time Kalman Filter $\quad 42$

5.2 Extended Kalman Filter $\quad 45$

$\begin{array}{lll}\text { 5.2.1 With Additive Noise } & 46\end{array}$

$\begin{array}{lll}\text { 5.2.2 With Non-Additive Noise } & 48\end{array}$

$\begin{array}{lll}5.3 & \text { Sigma-Point Kalman Filter } & 49\end{array}$

$\begin{array}{lll}\text { 5.3.1 With Additive Noise } & 50\end{array}$

5.3.2 With Non-Additive Noise

$\mathbf{5 . 4}$ Information Filter $\quad \mathbf{5 8}$

5.4.1 Extended Information Filter Measurement Information Calculation 60

5.4.2 Unscented Information Filter Measurement Information Calculation 61

$\begin{array}{lll}5.5 & \text { Mixed Kalman Filters } & 63\end{array}$

CHAPTER $6 \quad$ : SENSOR FUSION FORMULATIONS 64

$\begin{array}{llr}6.1 & \text { Coordinate Systems } & 64\end{array}$

6.2 GPS/INS Sensor Fusion Navigation Formulations $\quad 66$

$\begin{array}{lll}\text { 6.2.1 } & \text { 3-State Attitude Estimation } & 67\end{array}$

6.2.2 9-State Position, Velocity, and Attitude Estimation $\quad 69$

$\begin{array}{lll}\text { 6.2.3 Acceleration Vector Attitude Estimation } & 71\end{array}$

$\begin{array}{lll}\text { 6.3 GPS/INS + Magnetometer Sensor Fusion Formulations } & \mathbf{7 4}\end{array}$

6.3.1 3-State INS/Magnetometer Attitude Estimation $\quad 75$

6.3.2 2-State GPS/Magnetometer Attitude Estimation $\quad 76$

6.3.3 3-State GPS/Accelerometer/Magnetometer Attitude Estimation 77

6.3.4 3-State GPS/INS/Magnetometer Attitude Estimation 77

$\begin{array}{lll}6.4 & \text { Sensor Fusion Formulation Noise Assumptions } & 78\end{array}$

$\begin{array}{lll}\text { CHAPTER } 7 & \text { : SENSOR FUSION WITH SENSOR CALIBRATION }\end{array}$

$\begin{array}{lll}7.1 & \text { State and Parameter Estimation } & 82\end{array}$

$\begin{array}{lll}\text { 7.2 Random Walk Assumption for On-Line IMU Calibration } & 83\end{array}$

7.3 Sensor Fusion with On-Line Calibration $\quad \mathbf{8 5}$

7.3.1 9-State Position, Velocity and Attitude + 6- IMU Bias Estimation $\quad 85$

7.3.2 3-State Attitude + 6 IMU Bias Estimation $\quad 85$ 
CHAPTER $8 \quad$ : GPS/INS SENSOR FUSION PERFORMANCE COMPARISON $\quad 89$

8.1 Un-aided Low-Cost Navigation 89

8.1.1 Low-Cost Inertial Navigation 89

8.1.2 GPS Only Navigation 93

8.2 GPS/INS Sensor Fusion Comparison Study Flight Data Library 94

8.3 EKF and UKF Comparison for Loosely Coupled GPS/INS Sensor Fusion 96

8.3.1 Performance Evaluation Metrics 96

8.3.2 Simple Stochastic Sensor Modeling Approach 97

8.3.3 Performance Comparison Results 98

8.4 EKF and UKF Linearization Procedure Comparison 108

8.5 Nonlinear Information Filtering vs. Nonlinear Kalman Filtering 113

8.6 Position Error Smoothing and On-Line Calibration Position Drift Test 115

CHAPTER 9 : GPS/INS/MAGNETOMETER SENSOR FUSION AND CALIBRATION 117

9.1 GPS/INS/Magnetometer Sensor Fusion 117

9.2 Off-line and On-Line Low-Cost Magnetometer Calibration 118

9.3 GPS/IMU/Magnetometer Sensor Fusion Performance Comparison 119

$\begin{array}{lll}\text { CHAPTER } 10 & \text { : FAULT-TOLERANT ATTITUDE ESTIMATION } & 122\end{array}$

$10.1 \quad$ Redundancy and the Information Filter for Fault Accommodation 122

$\begin{array}{lll}10.2 & \text { Information Isolation } & 124\end{array}$

10.3 'Virtual Force-Field' Based Failure Detection and Identification 127

10.4 On-Line Calibration Based Failure Detection and Identification 129

$10.5 \quad$ 'Hard’ Sensor Failures 131

$\begin{array}{lll}10.6 & \text { 'Soft' Sensor Failures } & 139\end{array}$

CHAPTER 11 : CONCLUSIONS AND FUTURE DIRECTIONS 146 
11.1 Summary and Conclusion

11.2 Future Directions

REFERENCES 


\section{List of Figures}

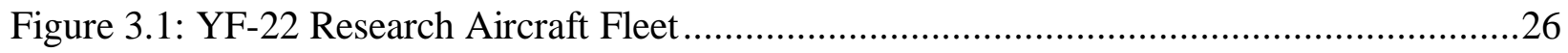

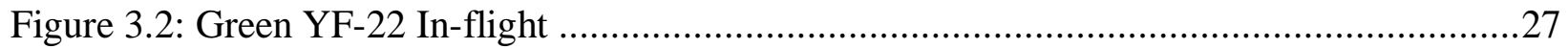

Figure 3.3: WVU YF-22 Formation Flight Hardware [reproduced with permission [68]]. ........29

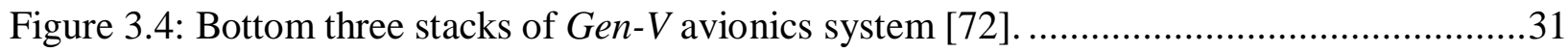

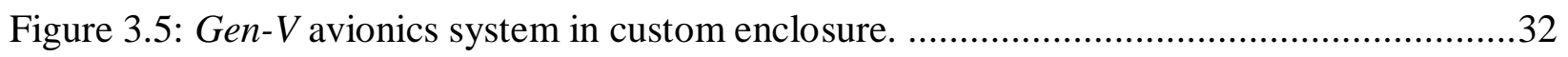

Figure 3.6: Gen-V avionics system block diagram (Figure Updated From [72]) ......................33

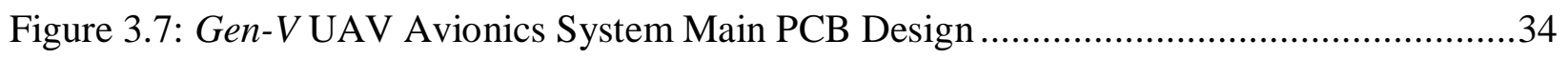

Figure 3.9: Goodrich VG34 Mechanical Gyroscope [74] .................................................36

Figure 3.10: GPS Track on Google Earth® Image Jackson's Mill Facility................................38

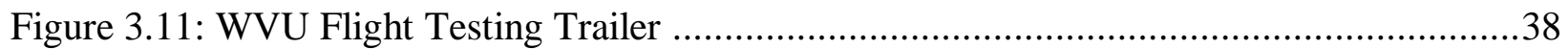

Figure 6.1: Aircraft body-axis convention. ...................................................................64

Figure 6.2: ECEF Coordinate Frame [image reproduced from Novatel [78]] .........................65

Figure 6.3: Local geodetic SWD coordinate system at WVU's Jackson's Mill flight testing



Figure 8.1: Low-Cost IMU Roll Estimation vs. Vertical Gyro Roll Measurement.....................90

Figure 8.2: Low-Cost IMU Pitch Estimation vs. Vertical Gyro Pitch Measurement ..................91

Figure 8.3: Position drift I and velocity drift (L) of Low-Cost INS compared to GPS..............92

Figure 8.4: A 2-D (x,y) map of low-cost INS position compared to GPS track. .......................92

Figure 8.5: An example of poor GPS position measurement. ........................................93

Figure 8.6 Flight data envelope with respect to speed and altitude. ....................................94

Figure 8.7: Distribution of aircraft attitude samples over 23 flights [87]. .............................95

Figure 8.8: Covariance tuning profile of the 9-State+6-Bias EKF and UKF (w/ non-additive

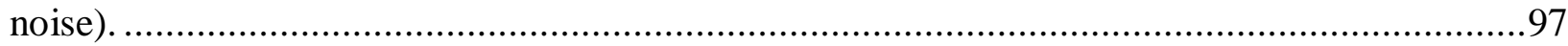

Figure 8.9:Non-Additive Noise GPS/INS attitude estimation performance over 23 for various sensor fusion formulations

101

Figure 8.10: Estimation performance variation between EKF and UKF over 23 flights for both types of assumed noise.

Figure 8.11: Pitch estimation compared with the vertical gyroscope measurement of different GPS/INS sensor fusion formulations using an EKF (assuming additive noise). 104

Figure 8.12: Roll estimation compared with the vertical gyroscope measurement of different GPS/INS sensor fusion formulations using an EKF. 105

Figure 8.13: Pitch Estimation EKF and UKF comparison for various GPS/INS formulations. .106 Figure 8.14: Roll Estimation EKF and UKF comparison for various GPS/INS formulations.... 107 Figure 8.15: EKF and UKF locally linearized attitude models over time..............................110

Figure 8.16: EKF and UKF 1 time step attitude magnitude prediction difference....................111

Figure 8.17: EKF and UKF 1 time-step covariance prediction magnitude difference. ..............112

Figure 8.18: Comparison of EKF and EIF over 23 flights........................................... 114

Figure 8.19: Comparison of UKF and EKF over 23 flights......................................... 114 
Figure 8.20: GPS Measurement error smoothing with 9-state + 6-IMU bias GPS/INS sensor fusion.

Figure 8.21: Position drift of the on-line IMU calibration filter during a 1 minute GPS outage.

Figure 9.1: Effect of magnetometer calibration on attitude Estimation, Off-Line and Off-Line with Off-Line

Figure 9.2: Comparison of 3-state GPS/INS, GPS/Mag., and INS/Mag. sensor fusion.

Figure 10.1: GPS/INS/Magnetometer sensor fusion block diagram. 124

Figure 10.2: Sensor information isolation for FDI. 126

Figure 10.3: 2-D Conceptual schematic of 'Virtual Force Field' FDI [98]. 129

Figure 10.4: Conceptual schematic of on-line calibration based FDI. 130

Figure 10.5: 'virtual force field' FDIA of natural GPS measurement quality degradation [98]. 132 Figure 10.6: Snapshots of 'virtual force field' detection during sensor failures. 133 Figure 10.7: 'virtual force-field' FDIA roll estimation performance during 315 second failures [98]. 135

Figure 10.8: 'virtual force-field' FDIA pitch estimation performance during 315 second failures. 136

Figure 10.9: Magnitudes of 'virtual forces' and associated failure detection threshold for each of the three information sources [98]. 137

Figure 10.10: Randomly generated rate bias 'virtual force-field' FDIA test. 138

Figure 10.11: FDIA of small pitch rate bias through on-line calibration tracking [98]. 140 Figure 10.12: FDIA of small pitch rate bias through on-line calibration tracking, 'turn-off' test.

Figure 10.13: FDIA of small magnetometer bias through on-line calibration tracking. 142 Figure 10.14: Sensor Fusion estimation error with and without FDIA during a growing magnetometer bias. 143

Figure 10.15: FDIA of growing accelerometer bias through on-line calibration tracking. 144 Figure 10.16: Sensor Fusion estimation error with and without FDIA during a growing accelerometer bias. 145 Figure B.1: Performance comparison of Joint vs. Dual Bias Estimation. .163 


\section{List of Tables}

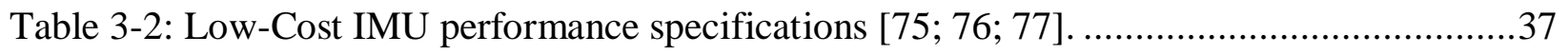

Table 8-1: GPS/INS sensor attitude estimation performance of Low-Cost INS and AVAE.......99

Table 8-2: Attitude estimation performance between EKF and UKF assuming additive noise....99

Table 8-3: Attitude estimation performance between EKF and UKF assuming non-additive noise

Table 9-1: GPS/INS/Magnetometers attitude estimation performance summary. ...................120

Table 10-1: 2-flight average fault-tolerant attitude estimation performance [98]...................131 


\section{List of Symbols}

\begin{tabular}{|c|c|c|}
\hline$a$ & $=$ & specific force in the aircraft body frame $\left(\mathrm{m} / \mathrm{s}^{2}\right)$ \\
\hline$B$ & $=$ & Aircraft body-axis \\
\hline c & $=$ & input vector to the observation functions \\
\hline $\mathrm{g}$ & $=$ & gravitational acceleration $\left(\mathrm{m} / \mathrm{s}^{2}\right)$ \\
\hline$E$ & $=$ & set of estimates from different information sources \\
\hline$F$ & $=$ & 'virtual force' \\
\hline $\mathrm{H}$ & $=$ & observation matrix \\
\hline$I$ & $=$ & Fisher Information Matrix \\
\hline $\mathrm{K}$ & $=$ & Kalman gains matrix \\
\hline 1 & $=$ & dimension of state matrix \\
\hline$L$ & $=$ & local navigation frame \\
\hline$N$ & $=$ & normal distribution \\
\hline$M$ & $=$ & Magnetometer readings \\
\hline$p$ & $=$ & roll rate $(\mathrm{deg} / \mathrm{sec})$ \\
\hline $\mathrm{P}$ & $=$ & covariance matrix \\
\hline$q$ & $=$ & pitch rate $(\mathrm{deg} / \mathrm{sec})$ \\
\hline$R$ & $=$ & rotation matrix \\
\hline$r$ & $=$ & yaw rate $(\mathrm{deg} / \mathrm{sec})$ \\
\hline $\mathbf{S}$ & $=$ & set on $\mathrm{N}$ sensors \\
\hline $\mathbf{u}$ & $=$ & input vector to the state-transition functions \\
\hline V & $=$ & velocity $(\mathrm{m} / \mathrm{s})$ \\
\hline$w$ & $=$ & weight vector \\
\hline $\mathbf{x}$ & $=$ & state vector \\
\hline $\mathbf{z}$ & $=$ & measurement \\
\hline$\alpha$ & $=$ & primary sigma-point scaling parameter \\
\hline$\alpha$ & $=$ & on-line calibration detection threshold \\
\hline$\beta$ & $=$ & secondary sigma-point scaling parameter \\
\hline$\beta$ & $=$ & covariance scaling during on-line calibration failures \\
\hline$\gamma$ & $=$ & compound sigma-point spread parameter \\
\hline$\theta$ & $=$ & pitch angle (deg) \\
\hline$\Theta$ & $=$ & calibration parameters \\
\hline$\kappa$ & $=$ & tertiary sigma-point scaling parameter \\
\hline$\lambda$ & $=$ & compound sigma-point parameter \\
\hline$\phi$ & $=$ & roll angle (deg) \\
\hline$\psi$ & $=$ & yaw angle (deg) \\
\hline$\chi$ & $=$ & sigma-points \\
\hline$\chi^{2}$ & $=$ & Chi-Square Test Statistic \\
\hline$\Omega$ & $=$ & 'force-field' detection threshold \\
\hline
\end{tabular}




\section{List of Acronyms}

\begin{tabular}{|c|c|c|}
\hline $\mathrm{AKF}$ & $=$ & Adaptive Kalman Filter \\
\hline CPU & $=$ & Central Processing Unit \\
\hline DCM & $=$ & Direction Cosine Matrix \\
\hline DGPS & $=$ & Differential Global Positioning System \\
\hline DR & $=$ & Dead Reckoning \\
\hline ECEF & $=$ & Earth Centered Earth Fixed \\
\hline $\mathrm{EDF}$ & $=$ & Electric Ducted Fan \\
\hline EKF & $=$ & Extended Kalman Filter \\
\hline EIF & $=$ & Extended Information Filter \\
\hline EMI & $=$ & Electro-Magnetic Interference \\
\hline FDI & $=$ & Failure Detection and Identification \\
\hline FDIA & $=$ & Failure Detection Identification and Accommodation \\
\hline FNN & $=$ & Fuzzy Neural Network \\
\hline GPS & $=$ & Global Positioning System \\
\hline IAE & $=$ & Innovation Based Adaptive Estimation \\
\hline IF & $=$ & Information Filter \\
\hline INS & $=$ & Inertial Navigation System \\
\hline IMU & $=$ & Inertial Measurement Unit \\
\hline $\mathrm{KF}$ & $=$ & Kalman Filter \\
\hline LLA & $=$ & Latitude, Longitude, Altitude \\
\hline LQE & $=$ & Linear Quadratic Estimator \\
\hline MEMS & $=$ & Micro-Electro-Mechanical System \\
\hline NN & $=$ & Neural Network \\
\hline PVA & $=$ & Position, Velocity and Attitude \\
\hline PWM & $=$ & Pulse Width Modulated \\
\hline $\mathrm{OBC}$ & $=$ & On Board Computer \\
\hline RF & $=$ & Radio Frequency \\
\hline RTAI & $=$ & Real Time Application Interface \\
\hline SPI & $=$ & Serial Peripheral Interface \\
\hline SPKF & $=$ & Sigma-Point Kalman Filter \\
\hline UAV & $=$ & Unmanned Aerial Vehicle \\
\hline UKF & $=$ & Unscented Kalman Filter \\
\hline UIF & $=$ & Unscented Information Filter \\
\hline WAAS & $=$ & Wide Area Augmentation System \\
\hline WLSR & $=$ & Weighted Least Squares Regression \\
\hline WMM & $=$ & World Magnetic Model \\
\hline
\end{tabular}




\section{Chapter 1 : Introduction}

\subsection{Problem Description}

The design of aircraft navigation systems is a continuously changing and active research area. The continued interest in studying aircraft navigation is largely attributed to the rapid advancement of instrumentation and computational power in terms of miniaturization, accuracy, and cost reduction. Most prominently, the Global Position System (GPS) along with sensors based on Micro-Electro-Mechanical-System (MEMS) technology have kept navigation research current. In order to take full advantage of the advances in instrumentation, algorithms that reject noise and optimally combine sensory information from multiple sources are needed. In addition, it is crucial to develop methods that allow for sensory components to extract calibration information from one another in real-time, and automatically reject poor measurements in order to exhibit fault-tolerance.

When considering the design of aircraft attitude estimation systems, the nonlinear nature of the problem poses a challenge that leave many interesting questions unanswered. These challenges include dealing with the nonlinearities associated within aircraft kinematics relationships, coordinate transformations, and individual sensor error dynamics. Toward addressing these challenges, it is critical to identify and verify through the use of experimental data, which type of nonlinear estimation filter is best suited for aircraft attitude estimation. In this study, two popular nonlinear Kalman filters, namely, the Extended Kalman Filter (EKF) and the Sigma Point Kalman Filter (SPKF) are compared in terms of estimation performance over a large and diverse library of Small Unmanned Aerial Vehicle (SUAV) flight data. In addition, in

order to approach the level of fault tolerance, approaches for improving the accuracy of 
individual navigation system components are needed, and more importantly must be verified experimentally. To address this, the effect of improving estimation performance when including a simple calibration model within the sensor fusion algorithm is experimentally shown. Finally, further address reliability, a fault-tolerant attitude estimation algorithm is presented, which is shown to detect and mitigate both abrupt sensor failures as well as sensor failures that slowly grow over time. In general, this research considers many of the current problems associated with the design of low-cost aircraft navigation systems, in the framework of employing sensor fusion.

Sensor fusion is defined as combining sensory information from multiple individual sources to obtain improved accuracy over what could be achieved using any of the individual components [1]. The International Society of Information Fusion (ISIF) defines the need for sensor fusion in today's world by stating:

"We are drowning in information but starved for knowledge. This level of information is clearly impossible to be handled by present means. Uncontrolled and unorganized information is no longer a resource in an information society, instead it becomes the enemy [2]."

This accurately conveys a research need within aircraft navigation systems. Specifically, with increases in computational resources and sensor miniaturization, the design methods and algorithms used to process navigation information become a central aspect of the design. This sentiment is reaffirmed by the USAF's Deputy Director of Avionics, when he stated "The challenge for the military research and development community is to vigorously exploit the simultaneous arrival of the GPS, the explosion in computational capability, and availability of the integrated and modular avionics architectures" in a 1992 article of The IEEE Aerospace and Electronics Systems Magazine [3]. This study is aligned with this direction, and seeks to employ sensor fusion to provide a better overall low-cost navigation solution for SUAVs. 
For the navigation systems of research SUAVs, the introduction of sensor fusion techniques addresses the common engineering design problem of balancing a system's performance with its cost, weight and size requirements. For the design of low-cost UAV platforms, the cost of high quality inertial navigation systems is generally too expensive. Recent advances in Micro-Electro-Mechanical-Systems (MEMS) technology have made low-cost Inertial Navigation System (INS) an appealing option; however the presence of sensor biases coupled with time integration results in a solution that quickly diverges, and therefore are hardly useable [4]. Specifically, the position drift of low-cost INS is proportional to the integration time squared of accelerometer bias, and the cube of rate gyroscope bias [5]. Toward solving this problem, the functional integration of measurements from a low-cost INS and a Global Positioning System (GPS) receiver can provide an accurate navigation solution with only lowcost components [6]. Additionally, other low-cost aiding sensors may be incorporated within sensor fusion navigation systems (e.g. Magnetometers) to provide additional information and improve accuracy and robustness [7].

This study employs sensor fusion to develop a low-cost SUAV attitude estimation system that exhibits high-accuracy and fault-tolerance to sensor component failures. This overall objective was broken down into three phases:

1. Obtaining an accurate fused attitude estimation solution. During this phase of the project, two popular nonlinear estimation techniques were applied to the aircraft navigation problem and were thoroughly compared for estimation accuracy using experimental flight data, namely: the Extended Kalman Filter, and the SigmaPoint Kalman Filter. 
2. Relying on sensor fusion to determine time-varying random calibration parameters. During this stage, on-line sensor calibration was investigated to estimate random residual sensor errors, and to achieve improved performance. In addition, a magnetometer calibration model was determined using a GPS/INS sensor fusion solution.

3. Implementing an algorithm architecture that provides a fault-tolerant attitude estimation solution. In this stage, the goal was to demonstrate the ability for the attitude estimation system to exhibit "graceful failures". For this phase, a nonlinear Information Filter, which is an alternative form of the Kalman Filter, was explored to provide a desired structure for sensor failure accommodation.

A major strength of this study is that it utilizes real SUAV flight data collected over several flight experiments, such that assessments are empirically derived. Additionally, within the recorded flight data, an attitude 'truth' measured by a high-quality mechanical gyroscope that is independent of sensor data fused together was collected. Therefore, attitude estimation performance between the various approaches considered was able to be accurately assessed. This is a break away from the traditional approach in the field, which has based assessments of performance upon simulation, and provides a practical assessment under real-world uncertainty conditions.

In addition to its theoretical contributions, this study also includes the hardware and lowlevel software design of a complex SUAV avionics package that is specifically designed for 
fault-tolerant flight control research. For the purposes of facilitating this sensor fusion study, a primary hardware design criteria of the SUAV avionics system was to allow for instrumentation flexibility. In this respect, the baseline components used for the sensor fusion study (MEMS IMU, and a GPS receiver) was augmented with magnetometers. Furthermore, the avionics system allowed for easy integration of tactical-grade navigation sensors (mechanical vertical gyroscope) in order to provide 'truth' data for sensor fusion evaluation. By incorporating a hardware design, this study provided a more complete educational experience.

Ultimately, the goal of this study was to design an attitude estimation algorithm that uses only low-cost components, exhibits a level of accuracy that is comparable to high-grade sensors, and is fault-tolerant to sensor failures. The algorithms designed are validated with experimental data, which is needed to provide clear evidence of reliability.

\subsection{Organization}

The remainder of this dissertation is organized as follows; Chapter 2 provides a review of the relevant literature, and Chapter $\mathbf{3}$ describes the experimental set-up as well as the development of the Gen-V SUAV avionics system that the author of this dissertation designed and built while at WVU. Chapter 4 is a succinct outline of the project objectives. Chapter 5 describes the nonlinear state estimation approaches considered in this study, then Chapter $\mathbf{6}$ describes the various sensor fusion formulations used in this study. Chapter 7 then discusses state and parameter estimation, and the resulting sensor fusion formulations with on-line calibration. In addition, this chapter discusses a magnetometer calibration approach that relies on GPS/INS sensor fusion.

Next, the results of this study are outlined in three chapters. First, Chapter $\mathbf{8}$ provides the results of the EKF/SPKF comparison study for GPS/INS sensor fusion both with and without 
IMU bias tracking. Next, Chapter 9 provides the results of including magnetometers, and the effects of the off-line and on-line magnetometer calibration approaches. Finally, Chapter 10 consists of the results of the fault-tolerant attitude estimation algorithm, and Chapter 11 summarizes conclusions of the study as well as future directions. 


\section{Chapter 2 : Literature Review}

Within the literature review, a brief historical overview of aircraft navigation is first discussed to provide the context needed to define the problem considered. This portion of the review concludes with an introduction to low-cost MEMS inertial navigation, a description of GPS, and brief review of utilizing magnetometers for additional attitude information. Next, some pertinent sensor fusion studies that are related to aircraft navigation and rely on nonlinear Kalman filtering are included are discussed. Additionally, the relevant topics within the field of nonlinear estimation are discussed. Specifically, the Extended Kalman Filter, the Sigma-Point Kalman Filter, and Information Filter are introduced. Next, some calibration techniques of aircraft navigation systems are reviewed, including approaches that consider the use of sensor fusion for calibration. Finally, because an avionics system was developed as part of this project, some recent developments in the design of research UAV avionics systems are reviewed.

\subsection{Low-Cost Navigation Sensors}

\subsubsection{Inertial Navigation Systems}

An inertial navigation system consists of a triad of orthogonal accelerometers and rate gyroscopes that are continuously updated using a computer to calculate the Position, Velocity and Attitude (PVA) of an aircraft over time [8]. By combining linear accelerometers with rate gyro measurements it is possible with Newton's first law to monitor the both the translational and rotational movements and completely define the trajectory of an aircraft from its origin of motion [9]. This is known as Dead Reckoning (DR), and provides a completely self-contained navigation solution. 
Historically, aircraft INS were mounted on a stabilized (i.e. non-rotating, gimbaled) platform [9]. In a gimbaled INS platform, two gyroscopes are used to cancel rotational precession, and the aircraft pitch and roll angles are monitored directly at the gimbal bearings. Velocity changes are monitored by directly integrating the linear accelerometers, since the platform keeps the accelerometers rotationally fixed within an inertial frame. The disadvantage of gimbaled systems is that they are large and heavy, and have expensive precision mechanical parts that could potentially jam (i.e. gimbal lock). Stabilized INSs are still in use today, but are not typically used for application within aircraft. Several textbooks [10;11] provide insight into stabilized INS and the issues confronted within these systems; however these systems are not pertinent to this study and will not be reviewed any further.

Today, strapdown INSs are more typically used within aircraft navigation systems. Within strapdown systems, the inertial sensors are fixed in the body-axis of the aircraft. This provides a light-weight solution, but requires additional computational resources, as well as sensors with a higher sensitivity range [9]. The need of additional computational power is a result of the fact the attitude must be constantly updated to correctly transform the accelerometers into the desired navigation frame while integrating for changes in position. Recent advances in MEMS technology have made low-cost strapdown systems available; however, due to sensor biases present on low-cost systems coupled with the time integration relationships needed for DR, the solution quickly diverges, and requires the use of aiding sensors to regulate the error growth [6]. To compensate for the error growth, aiding sensors, which are described next, can be used with the MEMS INS solution using sensor fusion. 


\subsubsection{Global Positioning System}

The Global Positioning System (GPS) was established in 1973 by the United States Department of Defense (DOD). It provides positioning by utilizing Time of Arrival (TOA) ranging, in which a network of satellites transmit a signal that includes precise transmission time and satellite location information [12]. Range information from multiple GPS satellites (at least four) provides information that is used to calculate the three-dimensional position of the GPS receiver within a Cartesian coordinate system that is within either a rotating frame or an inertial frame. The common designation of a Cartesian frame that rotates with the Earth's rotation is Earth-Centered Earth Fixed (ECEF), while an inertial frame is referred to as Earth-Centered Inertial (ECI) [12]. In this study, position and velocity information are provided by a GPS receiver in an ECEF coordinate system, which is more convenient for short duration SUAV because it rotates with the Earth.

In order to transform raw ranging information into an ECEF coordinate system, least squares solutions, Kalman Filtering, or iterative methods may be used [12]. For example, an iterative method for using the WGS 84 model to convert pseudorange data into ECEF frame is provided Bowring [13] .Today, however most commercial GPS applications provide position and velocity measurements in a convenient ECEF navigation frame, by relying on the DOD World Geodetic System 1984 (WGS 84) model [12], which accounts for gravitational irregularities due to the ellipsoidal shape of the earth. In this study, GPS information is obtained in an ECEF frame as computer by the receiver.

In order to more conveniently use the GPS position and velocity information, it is common to further transform the ECEF information into a local geodetic frame. A local geodetic frame specifies an origin within the ECEF frame, and provides a convenient 3-D frame for local navigation. Types of local transformations include: Latitude, Longitude and Altitude (LLA), 
East, North, Up (ENU), South West Down (SWD), among others [12; 8]. Within this study, the SWD coordinate frame is used.

Primary GPS error sources consist of measurement noise, propagation delay and nonsynchronous clocks [12]. Of these sources, the largest source of error is due to clock offset, or bias attibirbuted to actual clock bias and ionosphere delays [12]. This is the combined error due to an offset between the system time and the satellite clock, and the system time and the receiver clock. Additional GPS errors are attibuted to the geometry of the statellite constellation, and the multipath effect [14]. Put simply, the multi-path effect is caused by GPS signals being reflected by buildings and obstructions. Within this study, GPS information is used in a'loosely-coupled' and noise is considered at the GPS solution level and therefore individual noise sources are not modeled.

\subsubsection{Magnetometers}

Magnetometers are often used in navigation systems to provide additional information that consists of measurements of the magnetic field vector in the body-axis of the aircraft. Once calibrated for hard-iron and soft-iron deformations, this information is comparable to the World Magnetic Model (WMM) values of the magnetic field within ECEF coordinates [7]. In this setting, attitude information is available by determining the Euler rotations that are required to transform the body-axis magnetic field measurements to the WMM values that are referenced in a local coordinate system. 


\subsection{State and Parameter Estimation}

In general, many nonlinear estimation approaches attempt to build upon the Kalman Filter (KF), which is the optimal estimator for linear systems that are perturbed by, white, uncorrelated, and Gaussian noise. For a comprehensive review of nonlinear estimation, Chen [15] provides a summary of the field, and presents the derivation of the Kalman filter equations both from a least-square cost function and a Bayesian statistics point of view. Additionally, Arulampalam et al. [16] and Doucet et al. [17] both provide an overview of the different estimations methods for nonlinear systems developed since the widespread use of the Kalman Filter. Within this study, two popular nonlinear filtering strategies are considered and compared: Extended Kalman Filtering [18] and Sigma-Point Kalman Filtering [19]. In addition, a different form of the KF is explored, namely the Information Filter (IF), which can be used for nonlinear problems using either of the Extended [20] or the Sigma-Point [21;22] approaches. This review begins with the KF followed by its nonlinear extensions.

\subsubsection{Linear Optimal Kalman Filter}

The KF, which is also commonly referred to the Linear Quadratic Estimator (LQE), or the Kalman-Bucy Filter was derived by Rudolph E. Kalman in 1960 and published in 1961 by Kalman and Bucy [18], and has been hailed as a once in generation breakthrough that has had far reaching implications within the fields of control and estimation. The first cited application of KF was for navigation trajectory estimation for the Apollo program by Stanley F. Schmidt [23]. In simple terms, the KF handles uncertainty by computing states as a weighted average of predicted values and measured values. It has been proven to be optimal from the perspective of satisfying Bayes' rule [15], which is a fundamental result in statistics that provides an expression 
for the conditional probability of an event given another event, based on the likelihood, and prior probabilities of the individual events (2.1)

$$
P(A \mid B)=\frac{P(B \mid A) P(A)}{P(B)}
$$

The KF has also been derived to satisfy the recursive minimization of a cost function that minimizes the Mean Square Error of the estimates (i.e. error variance) [24]. This is interesting, given that in the field of estimation, optimization often is considered from a Bayesian perspective, or a least-squares perspective, and the KF has unique the property of satisfying optimality from both perspectives.

To meet its optimality conditions, however, several assumptions must hold [24; 23; 25]. First, the system must be linear. Second, the process noise and measurement noise must be uncorrelated with one another, white, zero-mean, and Gaussian noise. While the KF was breakthrough, meeting the linearity condition is not feasible for many practical applications. Therefore, approaches that consider nonlinear estimation are reviewed, that attempt to approximate this condition through use of two different linearization techniques.

\subsubsection{Extended Kalman Filter}

Perhaps the most traditional approach utilized for nonlinear estimation is the EKF [23], which consists of linearizing the system model around the most recent state estimates with a first-order Taylor Series expansion, and then using the KF equations. This approach was introduced shortly after the KF [18]. It uses an approximation of the nonlinear state transition functions by linearizing about the most recently estimated state vector in order to calculate the time-evolution 
of state error-covariance matrix, however it uses the intact nonlinear functions to propagate the state vector over time [25].

The EKF has known flaws, which are listed by Julier and Uhlmann [19] as - "difficult to tune, and reliable only if the system is nearly linear during the sampling time of the updates". To address the problems with the EKF several strategies have been developed to enhance performance, however many of these approaches are very application specific. For example, in some applications, EKF filter 'tuning', which refers to the process of assigning the design parameters that account for uncertainty, is often difficult. To address this, some adaptive techniques have been derived to 'tune' the EKF in real time [26]. This approach attempts to not only improve performance, but to prevent the filter from diverging [26]. As another example, Saisadek et. al. has considered fuzzy logic methods for adaptively tuning the uncertainty assumptions within EKF formulation on-line in order to increase performance and prevent solution divergence [27]. Additionally, studies have considered the use of Artificial Neural Networks (ANNs) to enhance EKF formulations for use in sensor fusion applications. Specifically for the application of GPS/INS sensor fusion, Chiang and Huang [28] as well as Jwo and Huang [29] explored the use of an ANN to compensate for the growth of INS errors during periods in which GPS measurements were not available. All of these methods have shown promise for enhancing EKF based GPS/INS sensor fusion, but are often designed to solve a very particular problem that the designer is facing, and are outside the scope of this study.

\subsubsection{Sigma-Point Kalman Filter}

In 1997, Julier and Uhlmann introduced the Unscented Kalman Filter (UKF) [19; 30]. The UKF has since been suggested to fall in a more generic class of filters called 'Sigma-Point' Kalman Filters (SPKF) [31]. The name Unscented refers the statistical transformation technique 
used to generate the 'Sigma-Points' in order to carry out statistical linearization. Additional variants of SPKFs that rely on other sigma-point selection techniques exist, including the Central Difference Kalman Filter (CDKF) [32]. The famous quote that of Julier and Uhlmann, [19] that is often cited as the motivation behind this UKF is that "it is easier to approximate a Gaussian distribution than it is to approximate an arbitrary nonlinear function or translation. [19]" The meaning behind this quotation is the fact that while the traditional (EKF) approach analytically linearizes the nonlinear functions, it is more intuitive numerically capture modes of Gaussian distribution that has been transformed through a nonlinear function $[19 ; 30]$. While the EKF relies on a first order linearization of the nonlinear functions, the UKF has been shown to obtain second-order or higher accuracies with respect the Taylor series expansion $[19 ; 30 ; 33]$. In this sense, the SPKF approach is viewed as relying on a Weighted Least Squares Regression (WLSR) for statistical linearization, and contributes less error associated with linearization, as pointed out be Lefebvre et. al [34]. van der Merwe et al. [32] developed a square-root implementation of the UKF algorithm (SRUKF). This approach avoids the need for a matrix square-root calculation at every discrete time step, by using sequential Cholesky updates. Additionally, it is been stated $[33 ; 31]$ that the order of computational complexity of SRUKF is the same as that of the EKF. This has been disputed in the literature [35]. However, differences in computational burden appear to depend on whether or not it is considered that the Jacobian partial derivatives are analytically determined offline or numerically computed at each filtering step. 


\subsubsection{Information Filter}

The Information Filter (IF) is an alternative form of the Kalman Filter [24; 20; 23], which is also known as the "inverse covariance Kalman Filter" [21]. For linear filtering applications, the IF is algebraically equivalent to the KF [21]. With the IF, instead of estimating the state mean and covariance, the measure of information of the state in terms of an information state vector and Fisher information matrix are estimated [22]. Simon in his textbook [23] points out that where the error covariance in the KF represents the uncertainty in the state estimate, the information matrix is a representation the certainty of the state estimate.

A practical advantage of the information form of the KF has been noted in terms of the possibility of computational efficiency [24;23], and some generalizations about special cases of initialization have been noted $[24 ; 23] \quad$ For example, an advantage of the information filter is the possibility of reduced computation in cases in which the dimension of the measurement of the system is larger than that of the state vector $[24 ; 23]$. This is due to the fact the in the information form, the largest inverse matrix that must be computer is the dimension of the state vector. Another interesting point noted in the literature is the case in which initialization of the uncertainty of the state is infinite. For the case of the KF, it is not possible to initialize the error covariance as infinite, while it is possible to initialize an information matrix to zero [23], the opposite is true when "perfect" knowledge of the initial state is known [24;23].

For nonlinear filtering problems both Extended [20]and Sigma-Point [22; 21] forms of the information filter have been developed. These forms have been cited as a useful framework for decentralized multi-sensor fusion architectures $[22 ; 21 ; 20]$. Specifically, Vercauteren and Wang developed a Sigma-Point Information Filter (SPIF) [21], and used a bank of them in a decentralized fashion for a target tracking problem. This algorithm showed to outperform 
similar approaches geared toward the same application, and exhibited a linear growth with the number of sensors used. On the contrary, the growth of centralized KF with an increasing measurement vector results in a computational growth that is associated with a larger matrix inversion. Lee [22] also formulated an Unscented Information Filter (UIF) and compared its performance to an Extended Information Filter (EIF) in a target tracking simulation. In this study, performance advantages for the target tracking problem were reported for the UIF over the EIF, and advantages with respect to the IF's computation complexity were pointed out in terms of providing "a natural means of integrating multiple sensor information".

\subsection{GPS/INS Integrated Navigation Systems}

The benefits of coupling Inertial Navigation Systems (INS) with Global Positioning System (GPS) solutions are well known [12; 6]. For example, Kaplan and Hegarty within their textbook [12], describe the coupling of GPS and INS information as "synergistic" since their own individual limitations are overcome when information from the two systems are combined. Specifically, the high frequency errors associated with GPS measurements is smoothed by inertial information, while the low-frequency drift associated with integrating an INS over-time is regulated by unbiased nature of the GPS solution [36]. This characteristic makes GPS/INS sensor fusion an appealing option for the design of low-cost UAV navigation systems.

\subsubsection{GPS/INS Sensor Fusion System Classification}

Within the field of GPS/INS sensor fusion algorithms there are some categories commonly used to classify the type of algorithm. Therefore one classification of GPS/INS algorithms is associated with the nonlinear estimation technique employed. A second category that classifies a 
GPS/INS algorithm defines the way in which the information is combined within the estimation filter. Specifically, this distinction is referred to a "loosely coupled" GPS/INS of "tightly coupled" GPS/INS [6]. A "tightly coupled" GPS/INS system uses the raw pseudo-range data required to solve for a GPS position and velocity measurement, and includes this information within the estimation filter. This allows for the information to be utilized even when the minimum number of required GPS satellites is in view of the antenna, and allows for improvements to GPS solutions through the estimation of GPS clock biases. On the contrary, a "loosely coupled" GPS/INS sensor fusion system directly utilizes the position and velocity measurements that are provided by a GPS receiver. This has the disadvantage of requiring an available GPS fix, and does not directly allow for enhancing GPS over filter operation, but is easier to implement. In addition, "loosely coupled" GPS/INS sensor fusion algorithms, are not hardware specific since GPS and INS are compartmentalized. This study considers the "loosely coupled" GPS/INS architecture.

\subsubsection{Previous GPS/INS Sensor Fusion Studies}

Within navigation, an early approach considered to meet the linearity condition required to apply linear Kalman Filtering for sensor fusion was to use a linearized model that approximates the time evolution of the errors of the states, known as an "error model" [37]. In this sense, both the error states mean and covariance are propagated with the linear model based on a perturbation analysis that assumed low errors. For example, Maybeck [37] utilized a nine-state INS error model, which was simplified version to that formulated by Pinson [38] in order to combine GPS and IMU data. This particular model considers a triad of position, velocity and attitude error states. A complete derivation of the Pinson error model formulation is described 
within [8]. For the Pinson error model, the small perturbation assumption is used to derive a linear model that approximated the time variation of the errors of the states. Larger order state error models are utilized within [39]. This study, however, the nonlinear kinematic equations, and use linearization where needed in the nonlinear form of the KF.

Many researchers have carried out comparative studies between EKF and SPKF for application within GPS/INS integration in aerospace vehicles [31; 32; 40; 41]. However, these studies were carried out in simulation, and presented some contradictory findings. For example, van der Merwe, Wan [31; 33]' and Julier [31] found that Sigma-Point Kalman Filters provided superior estimation, improved bias estimation, and offered more resilience during a loss of GPSsolution, as compared to EKF for the application of GPS/INS integration in a high-fidelity simulation of an MIT autonomous helicopter. In this study, estimated obtained by both an EKF and UKF are shown using measured flight data, however all performance comparisons are drawn with respect to results obtained from a simulation environment. This is due to the fact that an independent source of attitude truth was not available onboard the autonomous helicopter throughout the flight tests. Furthermore, it is unclear if the exact uncertainty assumptions were used between the two estimators. Specifically, it appears that results obtained with a 'fine-tuned' EKF designed by MIT researchers were compared to estimates obtained with SPKF designed by van der Merwe, et al. [32]. Because it is well know that changes in assumed error covariance may greatly impact performance, this could have impacted the results. Within another effort [40], Crassidis provided insight towards resolving an issue of obtaining a quaternion unity norm estimate. In addition, Crassisdis' results showed marginal improvement in the SPKF over the EKF for GPS/INS sensor fusion, but highlighted that performance improvements were most evident when large initialization errors are present. Interestingly, Wendel et. al. [41] reported that 
for a tightly coupled GPS/INS navigation system simulation that considered the specifications of different grade inertial sensors and various forms of GPS satellite signals, that EKF and SPKF provide identical estimation performance. Therefore, not all the questions regarding EKF vs. UKF for this application have been answered, and an experimental analysis would be useful to supplement some of the recent simulation studies in the field.

EKF and UKF comparison have also been carried out for related applications [35; 42]. For example, St. Pierre and Ing [35] compared simulated ground vehicle position estimates provided by GPS/INS sensor fusion using both EKF and UKF. Within their study, it was determined that UKF position estimates were slightly more accurate than EKF when GPS is available; however, interestingly it was claimed that when no GPS is available, the EKF outperformed the UKF. In addition, St. Pierre and Ing [35] contended that EKF requires far less computation time than UKF. Air Force researchers [42] compared EKF and UKF for the spacecraft application of angles based relative navigation. This study the UKF only sometimes provides performance improvement over EKF, however at levels of improvement that are often not significant. This study, also acknowledged a drastic increase in the computation time of the UKF over the EKF, and also stated that using Cholesky matrix decomposition for the square root operation caused filter divergence in certain instances where the Matlab® square root matrix function did not.

\subsubsection{GPS/INS + Other Aiding Sensors based Integrated Navigation}

In addition to GPS/INS information, several studies have included additional aiding sensors. A popular aiding sensor suite included for attitude estimation is a triad of magnetometers mounted in the body-axis of the aircraft $[43 ; 44 ; 45 ; 46 ; 7]$. For example, Oh and Johnson [44] presented a low-cost navigation system that fused information from GPS/INS as well as a suite of magnetometers. Within this study, an EKF and UKF formulations were 
compared within simulation, and it is concluded that EKF and UKF each have the own advantages and disadvantages. Specifically, it was concluded that the UKF is computationally more demanding, and EKF requires the calculation of Jacobians for linearization which may not be trivial. In addition within Oh and Johnson's study, they confronted the issue of dealing with information that is collected at different sampling rates by employing sequential measurement update procedures. Within another study by Changey et. al. [45], an attitude only estimation filter is designed using magnetometer data. This study relied on a "Mixed Extended-Unscented Filter". Within the mixed filter, the UKF measurement update procedure is coupled with an EKF time-update procedure. This mixed approach is advantageous if either the time-update or measurement update is linear. Crassidis and Lightsey [46] also demonstrated attitude determination on of a spacecraft using GPS and magnetometer data. Within this study, the ambiguities of GPS only attitude determination was resolved by using the phase difference of measurements obtained with two GPS antenna was fused with magnetometer information within simulation. Finally, Guo et al [7] discussed an EKF calibration procedure of the hard iron and soft iron distortion of magnetometer information. This allows not only for magnetometer data to be used to improve attitude estimates, but also for GPS/INS data to aide in the calibration of magnetometers.

\subsubsection{Sensor Fusion Based Calibration}

Some studies consider the use of nonlinear estimation algorithms that involve sensor fusion for the aim of calibration. A traditional approach involves including time-varying calibration parameters along with original system states into the estimator to form a dual state and parameter estimator [25; 23]. Within GPS/INS sensor fusion, another approach toward adaptive filtering is to consider on-line variation of the process-noise and measurement-noise 
covariance matrices [47; 48; 49]. For example, Mohamaed and Schwartz [48] considered Adaptive Kalman Filtering (AKF), which first ruled out the applicability of Multiple Model Adaptive Estimation (MMAE) over Innovation Based Adaptive Estimation (IAE). In the IAE approach, a residual sequence is monitored over a fixed window, and is used to derive an adaptation parameter to either update the process-noise matrix on-line or measurement-noise covariance matrix on-line. In this study, they showed that by adaptively tuning either the process-noise or measurement-noise matrices, that navigation state estimation could be improved. Similarly, Ding et al. [47] considered an AKF that used the residual between the GPS and INS predicted states over a window to update the process-noise and measurement-noise online. Hu et al [49] considered AKF for GPS only navigation in land vehicles with the use of scale factor that relied on fading memory to alter the process-noise covariance matrix. Hu showed improvements with respect to position tracking error as compared to a conventional EKF.

Other studies have considered on-line calibration [50; 51]. For example Kim et. al. [52] designed a two-stage adaptive EKF for GPS/INS fusion, where the goal of the filter design was to on-line calibrate IMU sensor biases, as well as update the process-noise and measurementnoise parameters. In the study, the detection of imposed fault biases was demonstrated to show better accuracy over a non-adaptive EKF. El-Diasty and Pagitaka [51], provide further insight on stochastic modeling for considering random errors present within inertial sensors an on-line calibration. Fox [53], used a SPKF for the problem of IMU sensor calibration. In this study, a robot was used to generate dynamic motion, and based on the known robot motion "ideal" sensor measurements were derived and compared to the raw measurements to formulate a nonlinear Parameter Identification (PID) problem. In this approach calibration parameters were successfully determined. 


\subsection{Sensor Fusion with Failure Detection and Accommodation}

Many studies in the literature have focused specifically on Kalman Filter architectures that a derived to support fault-tolerance of sensor failures with respect to navigation related applications $[54 ; 55 ; 56 ; 57 ; 58 ; 59 ; 60]$. In 1987, Kerr [54] provided a comprehensive review of various FDI approaches used in KF applications for navigation systems, and presented a decentralized approach that used local filter for individual sensor processing and a master filter to combine information and used Gaussian hypothesis testing for failure detection. Carlson [58; 57], further developed a decentralized KF approach [58] as well as a square-root implementation [57], named a "Federated" KF that could provide a globally optimal solution if each of the local KF were reset with the estimates from a global filter, or has the option of providing heightened fault-tolerance by allowing each of the local filters to maintain their own 'a posteriori' mean and covariance. These approaches considered multiple linear systems, however more recently some authors have applied the federated filter for fault-accommodation on nonlinear problems. For example, in 2011, Bae, Yoon and Kim [60] presented a federated UKF for application to a numerical simulation of satellite attitude estimation using a gyroscope and redundant star trackers. In their implementation, FDI was achieved with a sensitivity factor that is related to a Chi-square hypothesis test, and estimates from local filters were removed upon detection so that the global estimate was not affected. Duan et al [61] used an expert system to modify the information sharing coefficients used to scale the error-covariance matrices estimated by local filters before fusing in a master filter. This has the advantage of obtaining estimates closer to the global optimum when the sensors are healthy, while maintaining fault-tolerance. Fauske et al [55] considered the application of an Autonomous Underwater Vehicle and used a bank of KFs that each fused a subset of the available aiding sensors that provided partially redundant 
information, and a master filter that used all the information in order to isolate sensor and accommodate for failures. In this application however, all of the KFs relied on the same IMU, and only the aiding sensors failures were considered. FDI was achieved using an overlapping covariance ellipsoid test. Mirabadi et al [59] compared Chi-Squared and KF residual test based FDI for application of both 'soft' and 'hard' sensor failures in train navigation, and found the residual test to be less sensitive to failures and not recommended for soft failure detection.

\subsection{Research SUAV Avionics System Design}

Although commercially available autopilots have been extensively used for UAV research applications, the need to evaluate advanced flight control designs often leads researchers and institutions to develop custom UAV avionic systems [62]. These custom system architectures vary depending on their operational requirements, and due to practical constraints such as size, weight, power consumption and expense. For example, NASA Langley's Airborne Subscale Transport Aircraft Research Test bed (AirSTAR) program uses 'dynamically scaled' jet-powered vehicles that are equipped with a customized avionics system for a research program focused on improving aviation safety [63]. The AirSTAR system maintains most of computational resources at a ground station (e.g. rack mount computers in a mobile ground station) which receives measurements from Radio Frequency (RF) downlink, and sends control commands through an RF uplink [63]. The onboard computation resources in this architecture are limited to collecting, multiplexing, and sending out the data stream, while the ground station computers execute flight control algorithms. This system architecture has the benefit of relying on computational power that is not limited by size, weight, power consumption, and is easy to upgrade, but could experience problems due to communication interference, which may result in 
information latency and/or packet loss. Another example of a custom UAV avionics system is the FCS20, which was developed at the Georgia Institute of Technology. This system is based on a "Digital Signal Processing/ Field Programmable Gate Array board which has a credit card sized form factor" [64]. Currently, a version of the FCS20 system has since been commercialized and is for sale by AdaptiveFlight, Inc. A different approach to UAV flight control instrumentation is use of indoor flight testing facilities in which high speed cameras and laser based systems are used to measure UAV dynamics. For example, the MIT utilizes an indoor flight testing facility in which the laboratory environment known as Real-time indoor Autonomous Vehicle Test Environment (RAVEN) is outfitted with the necessary equipment for measuring the UAVs dynamic states [65]. The indoor test facility approach has the clear advantage of being able to rapidly perform complex flight control tests on a large variety of lowcost platforms. Specifically, because no instrumentation is required on the actual UAV platform, off-the-shelf hobbyist "ready-to-fly" airplanes can be used for flight controls research. However, with respect to up-front costs, this approach likely exceeds the affordability limitations of many within of the research community, and does not provide a solution implementation outside of the research environment. UAV research programs often lead to the development of customized instrumentation.

WVU researchers have been involved in UAV research and the development of UAV electronic payloads since 1997. Developing customized avionics systems for flight controls research started with basic data acquisition [66] but has since evolved to a full range of flight control applications. For example, a system was developed for WVU's jet-powered YF-22 research aircraft used for validating GPS-based formation flight control laws $[67 ; 68 ; 69]$ and has since been used for evaluating fault-tolerant flight control laws [70]. In more recent efforts, 
WVU researchers have also developed a small autopilot as part of a Modular Avionics Platform (MAP) that weighs only $3 \mathrm{oz}$. and provides actuator driving, radio telemetry, GPS and IMU data [71]. Within this study, the development of a new system Gen-V ( $5^{\text {th }}$ Generation at WVU) that is based upon the lessons learned throughout WVU's decade of UAV avionic system development as well as adapting the latest technological advances to address new research requirements is described. The design of this new system is discussed in the Chapter 3: Experimental Set-Up of this dissertation. 


\section{Chapter 3 : Experimental Set-Up}

This section of the thesis describes the research UAVs, the onboard electronic instrumentation payloads including the design of the Gen-V avionics system [72], and the flight testing operations.

\subsection{WVU YF-22 Research UAV}

The flight data used in this study was collected onboard the fleet of WVU YF-22 research UAVs, as shown in Figure 3.1.

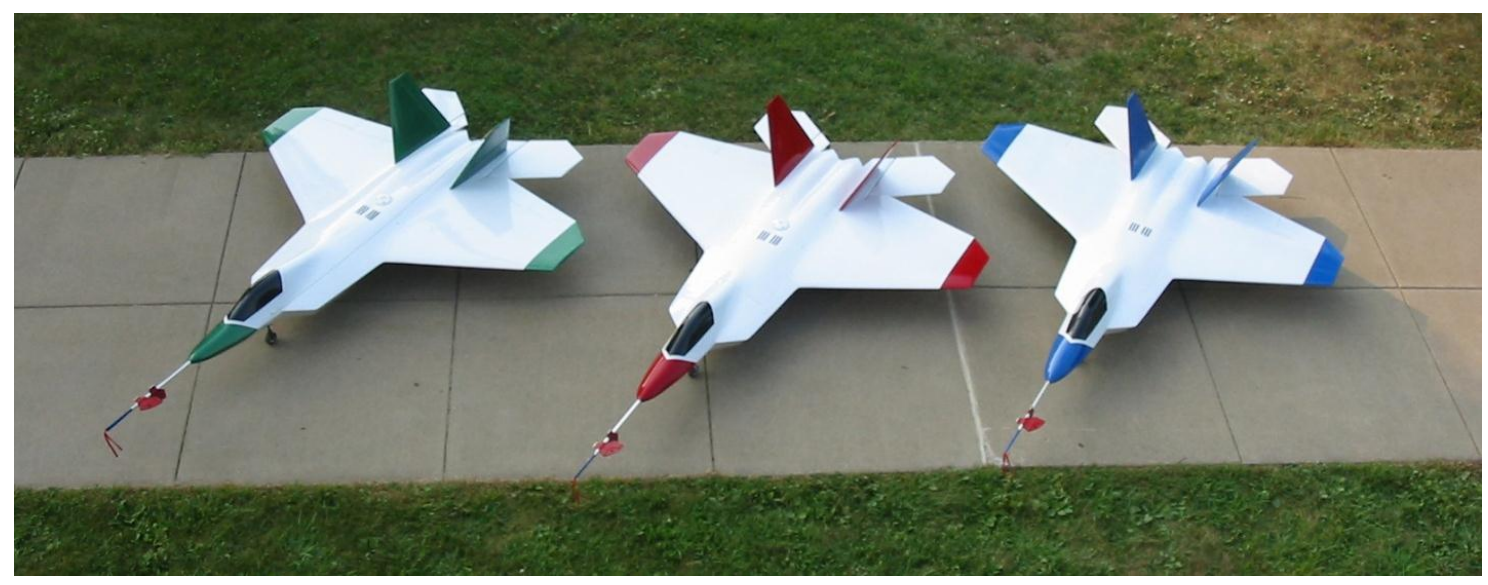

Figure 3.1: YF-22 Research Aircraft Fleet

Each of the YF-22 research aircraft has a length of approximately $8 \mathrm{ft}$., and a wing span $6.5 \mathrm{ft}$.

The take-off weight was nominally $50 \mathrm{lbs}$., which included $12 \mathrm{lbs}$. of electronic payload and nearly $5 \mathrm{lbs}$. of Jet-A fuel. Over the years of operation, two different propulsion systems were used within the YF-22 first a RAM-100 miniature turbine that produces 28 lbs. of static thrust, and more recently a Jet-Cat P-120-SX model jet engine that produces $30 \mathrm{lbs}$. of static thrust has been retro-fitted in the aircraft. An in-flight picture of the Green YF-22 is shown in Figure 3.2. 


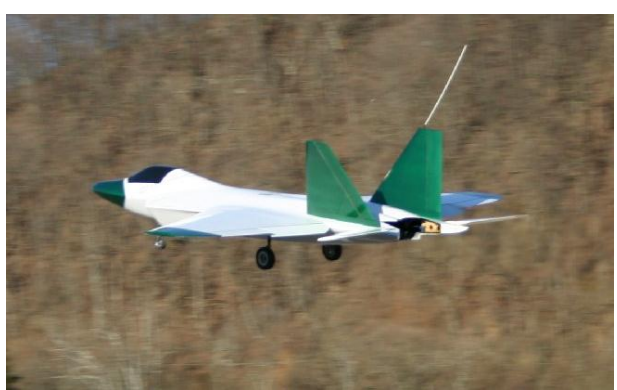

Figure 3.2: Green YF-22 In-flight

The YF-22 has been operational since 2002. Over the past few years, the fleet was updated with trailing link nose gears to improve runway steering quality and new model jet engines. The design of the new electronic payload was part of this effort, and is described in the next section of this Chapter.

\subsection{Onboard Computer and Sensor System}

\subsubsection{Design Requirements}

While the focus of this dissertation is sensor fusion navigation algorithms, an avionic system named Gen- $V$ was also designed as part of this graduate project, in order to fulfill the needs of several research areas at WVU. The avionic system that designed and built was a natural evolution of the existing avionics systems that were designed by previous WVU researchers [68; 71]. Some of the high-level design requirements for the system, which covers several typical SUAV research missions, are tabulated by Gu et al. [71] , and were reduced to the following list of specific avionics requirements. This list used as the high-level design criteria for the $G e n-V$ system: 
- High quality navigation measurements with a fast update rate.

- Ability to monitor ground R/C pilot commands.

- Ability to individually control each control surface, and inject pre-specified surface failures, and autonomously control surfaces alone or in conjunction with a ground pilot.

- A sufficient amount of computational power in order to allow for complex realtime control schemes.

- Low size, weight and power consumption.

- Telemetry uplink/downlink.

Before, the Gen-V design is described; the WVU YF-22 Formation Flight Avionics System (FFAS) is described. This is appropriate because a majority of flight data used in this dissertation for the GPS/INS comparison study was acquired by this system. In addition, this is the system that the design of the Gen- $V$ avionic system was rooted.

\subsubsection{YF-22 Formation Flight Avionics System}

The YF-22 formation flight avionic system consists of an On-Board Computer (OBC) based on a PC-104 stackable system that consists of a 300 MHz Central Processing Unit (CPU) board, a power supply, and a data acquisition module with 32 A/D channels [68]. The sensor suite features a high-quality mechanical vertical gyro for direct measurement of the vehicle's roll and pitch angle, a GPS receiver, and a Crossbow MEMs IMU [68]. In total, 3 different Crossbow 
IMU's with slightly different specifications were used in this avionics system, and a full description of them is included later in this Chapter.

The FFAS provides six independently controllable channels that allows for multi-controllable channel configurations. Among the six controllable channels, actuator failures can be 'injected' during flight maneuvers, and the vehicle can be either flown under manually piloted control, autonomously, or partially autonomous [71]. A layout of the current YF-22 vehicle and payload hardware configuration are shown in Figure 3.3.

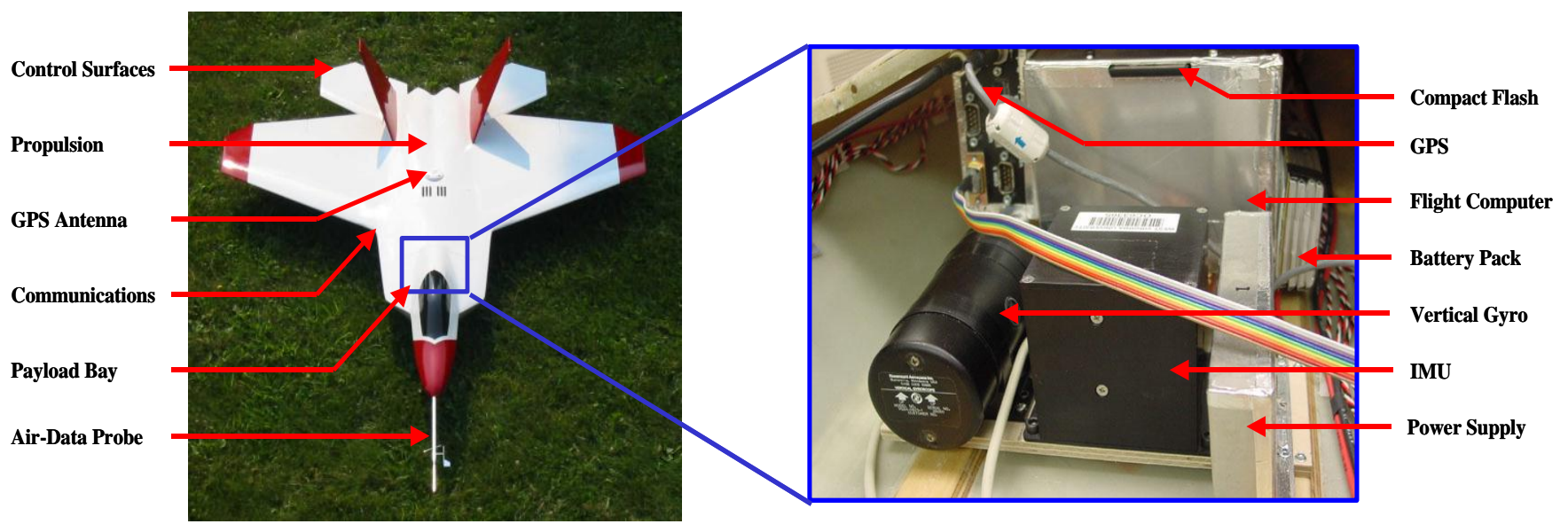

Figure 3.3: WVU YF-22 Formation Flight Hardware [reproduced with permission [68]].

For this study, a diverse library flight data that was collected with the formation flight hardware over the past several years was used to design and validate sensor fusion algorithms. In addition, flight data collected with the newly designed Gen-V system was also utilized. 


\subsubsection{Gen-V Avionics System Development}

The development of the Gen-V avionics system is described in a 2009 AIAA GNC conference Proceedings article [72].

\subsubsection{System Design Architecture}

The Gen-V system consists of a stack of four Printed Circuit Boards (PCBs), and is the $5^{\text {th }}$ generation of avionics system custom developed for SUAVs at WVU. The $1^{\text {st }}$ (top) board of the stack is a custom developed board that includes a GPS receiver, indication Light Emitting Diodes (LED), a compact flash card reader, and multiple microSD data recorders. This board design was carried out by Frank Barchesky at WVU in order to improve upon the original design and include the GPS receiver inside a single enclosure in the system. The $2^{\text {nd }}$ board in the stack is Diamond System's Athena II general purpose Single Board Computer (SBC) that features 16 channels of 16-bit integrated data acquisition, and four serial ports. The $3^{\text {rd }}$ board is a standard PC/104 power supply card that also provides two additional serial ports. Finally, the $4^{\text {th }}$ (bottom) board of the stack is a custom developed PCB that serves as the main sensor interface, as well as a signal distribution controller that includes the logic gates and duplexers necessary for allowing the aircraft to operate in different control modes, which are described later in this Chapter. The custom interface and signal distribution board was designed by the author of this dissertation.

The overall dimensions of the Gen-V system in comparison to the YF-22 FFAS resulted in over a 50\% reduction in total volume, and the total weight of the system is approximately $3 \mathrm{lbs}$., with an approximate 5 lbs. weight saving in comparison to FFAS. A picture of the bottom three stacks of the Gen-V system is shown in Figure 3.4. 


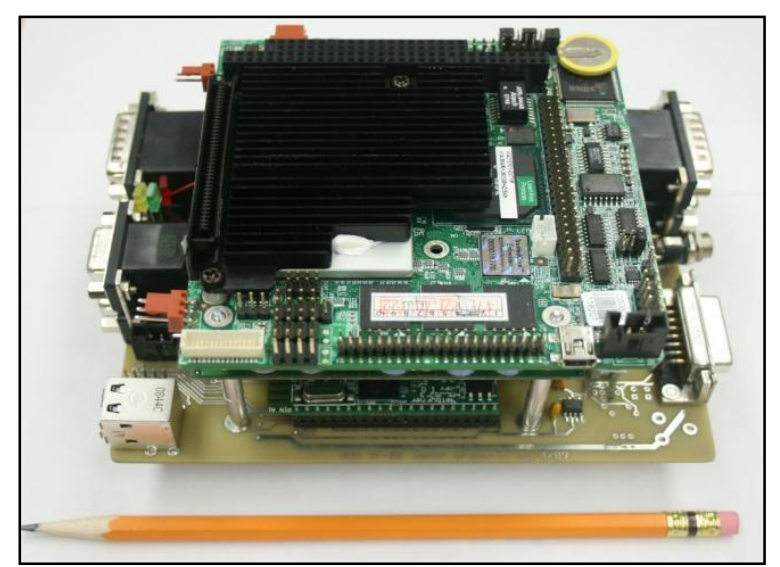

Figure 3.4: Bottom three stacks of Gen-V avionics system [72].

A Freescale ColdFire MOD-5213 ${ }^{\circledR} 66 \mathrm{MHz}$ microprocessor is integrated into the bottom custom PCB and is used to handle much of the low-level communications workload of the overall avionics system. Netburner's MOD-5213 Integrated Development Environment provides a real-time operating system that allows for multi-level interrupt-driven task prioritization. The MOD-5213 tasks include interfacing with a digital MEMS ADIS-16405 IMU, reading ground pilot input command signals, and writing actuator commands based on the specific flight control laws that are executed on the General Purpose Computer (GPC). To interface with the ADIS16405 IMU the MOD-5213's serial peripheral interface (SPI) module is used. Diamond System's $800 \mathrm{MHz}$ Athena II general purpose computer with integrated Analog-to-Digital conversion serves as the main flight-computer. On the main computer, a total of six serial ports are available for communicating with various devices. Of the six serial ports, two are configured for communication with the MOD-5213 microprocessor, while an additional two are utilized to interface with a GPS receiver and a RF modem respectively. Another serial port is directly linked to a micro-SD serial data recorder to serve as a system "black-box", and the remaining serial port connects with sensors located on a nose probe interface board. Figure 3.5 shows the complete system stack in a custom designed enclosure, which was CAD designed by fellow WVU graduate student Frank Barchesky. 


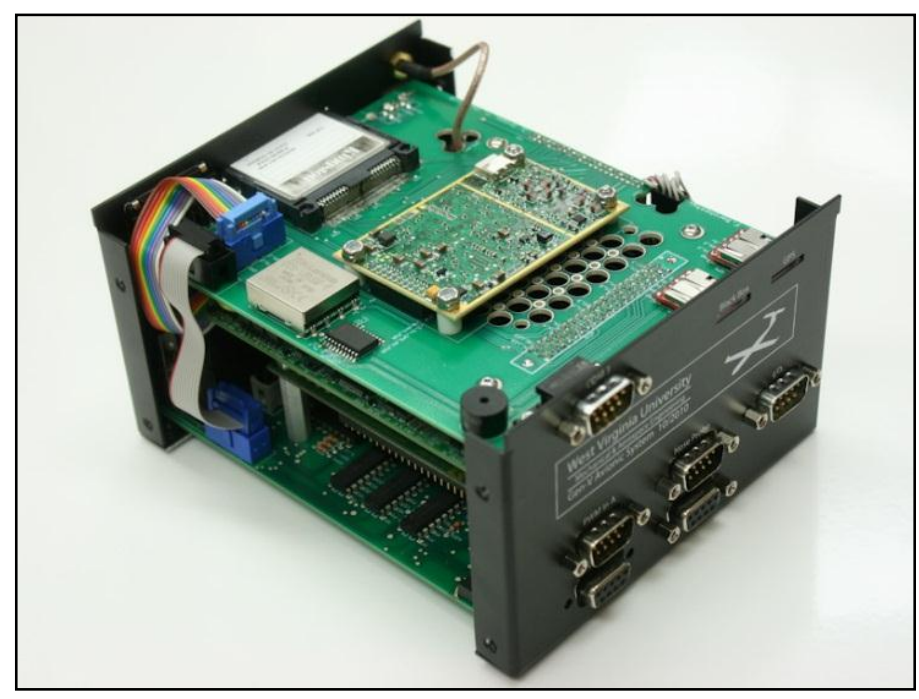

Figure 3.5: Gen-V avionics system in custom enclosure.

Sensors outside of the main system enclosure consist of nose probe sensors, and control surface deflection indicators. This includes potentiometers that measure control surface deflections. Additional analog sensors include vaned potentiometers that measure the angle of attack and sideslip flow angles, a thermister that measures air temperature, and pressure transducers that measure both the static and dynamic pressures. Within the YF-22, the angle of attack and sideslip measurement vanes and the Pitot tubes are part of a high-quality SpaceAge ${ }^{\circledR}$ Probe. Figure 3.6 shows the Gen-V system block diagram. 


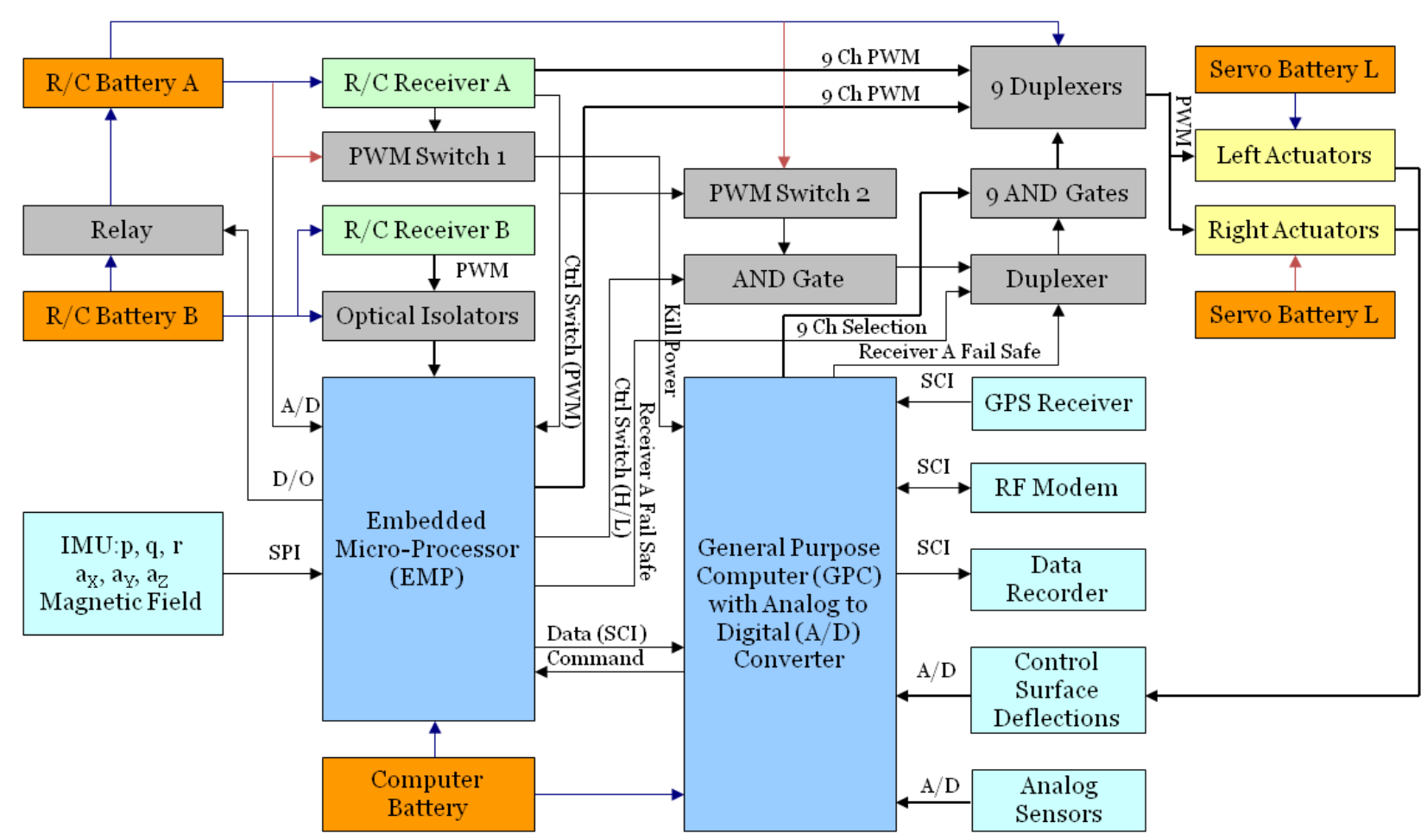

Figure 3.6: Gen-V avionics system block diagram (Figure Updated From [72])

The color-coded categories indicated in Figure 3.6 include, processors, batteries, sensory devices, and logic hardware for control signal routing. The batteries include NiMh R/C batteries, Lithium Polymer computer batteries and NiMh servo batteries. The architecture illustrated in Figure 3.6 is designed to allow for two pilot inputs to provide a level of redundancy. This was designed to limit the number of single-points-of-failure within the system. Another more simple architecture that only features a single R/C receiver was also designed. Figure 3.7 shows a top view of the bottom custom designed PCB. 


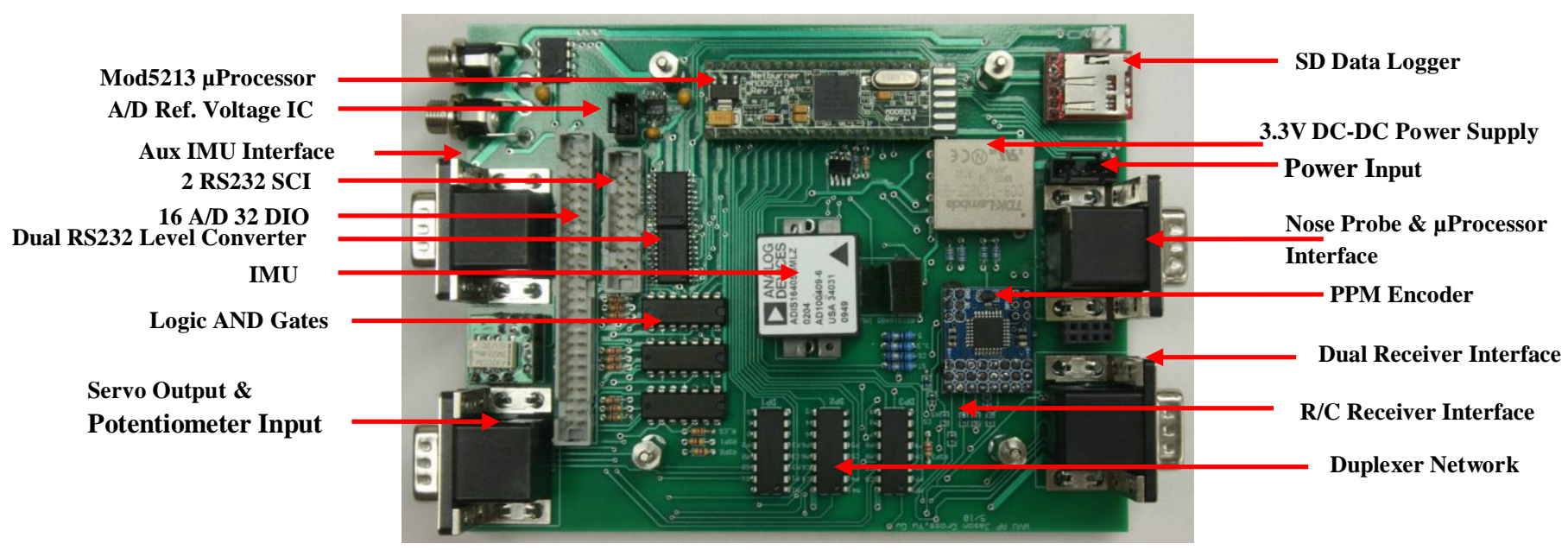

Figure 3.7: Gen-V UAV Avionics System Main PCB Design

\subsubsection{Control Command Signal Distribution}

In order to monitor ground pilot commands a 16-bit general purpose timer on MOD-5213 microcontroller is utilized to interpret the timing information of the command signal. First, the eight individual pilot Pulse Width Modulated (PWM) commands from the R/C receiver are multiplexed into a continuous stream of sequential square-waves, which is known as the PulsePosition Modulation (PPM) format, using an off-the-shelf PPM encoder. By monitoring the ground pilot commands, the Gen-V system allows for the possibility of human pilot-in-the-loop control, where the on-board controller and ground pilot collaborate.

For autonomous operation, the system also has the capability producing nine PWM signals based on commands produced by the on-board control algorithm. To switch between autonomous and manually piloted models, a second general purpose timer on the MOD-5213 is used to reading the system "Mode Switch" that is controlled by the ground pilot and determines whether the ground pilot or the on-board software has control over the UAV. Finally, a third timer is used to monitor a pilot PWM "Kill-Switch", which provides the ground pilot the ability 
to turn off the instrumentation payload in the event that Electro Magnetic Interference (EMI) interrupts the Radio-Frequency (RF) link between the R/C transmitter and the aircraft.

\subsubsection{Fault-Tolerant Architecture}

In the Gen-V system a total of nine channels can be controlled independently by either a ground pilot or autonomously by the on-board software, where each of these channels uses the same logic design that was used on the 6 controllable channels of the FFAS [68], however it is slightly modified to include additional redundancy. Nine independent channels allow independent control of typical channels such as throttle, all the conventional control surfaces (rudder, aileron, and stabilator) as well as independent control of the flaps. However, the nine available channels are generic in that they can be utilized in application specific configuration. For example, the Gen-V system is the primary avionics system for a new WVU test-bed that will use propulsion assisted control. This aspect of the system uses both hardware and software components to provide a two-tiered signal control system.

\subsubsection{Real-Time Operating System}

The onboard Operating System (OS) utilized is a Linux kernel patched with Real-Time Application Interface (RTAI). With the approach outlined by Campa [73] a RTAI target has been be implemented so that algorithms developed in the Matlab ${ }^{\circledR}$ and Simulink ${ }^{\circledR}$ may be compiled to create real-time executable using the Real Time Workshop ${ }^{\circledR}$. Currently, the real-time operating system (RTOS) is bootable on a Compact Flash (CF) card through an IDE adapter that is on the top custom PCB. The current target environment is Linux kernel 2.6.9 patched with RTAI 3.2, and WVU researcher Dr. Srikanth Gururajan is the RTOS developer. 


\subsection{Sensor Fusion Truth Data}

Attitude truth measurements used for performance comparison of various sensor fusion attitude estimation algorithms in this study are provided by a Goodrich ${ }^{\circledR}$ VG34 mechanical gyroscope which was carried alongside the low-cost sensor fusion components, as shown in Figure 3.9.

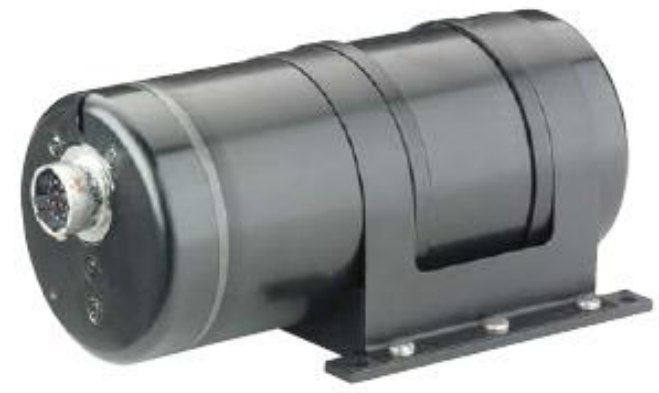

Figure 3.8: Goodrich VG34 Mechanical Gyroscope [74]

The VG34 mechanical gyroscope has a $\pm 90^{\circ}$ sensitivity on the roll-axis and $\pm 60^{\circ}$ sensitivity on the pitch-axis. The signal outputs are analog voltages that were acquired over a 10-volts at a 16bit resolution. This corresponds to a resolution of 0.0183 degrees on the pitch channel, and 0.0275 degrees on the roll channel, the vertical gyroscope has a self-erection system, and s reported accuracy within $+/-0.25^{\circ}$ of the true vertical [74]. Prior to each flight, the mechanical gyroscope is leveled with a bubble level for the duration of three minutes. The disadvantage of using this sensor within SUAV applications is that weighs $1.3 \mathrm{lbs}$., which may be a significant portion of the payload capacity. It also requires 28-volts, which is above the voltage level of many small batteries, and causes a lot of EMI.

\subsection{Sensor Fusion Source Data Performance Specifications}


While the Gen-V system was developed as a part of this project, flight data collected onboard thee WVU YF-22 with several sensor payloads were utilized in order to diversify the number of inertial sensors considered in this fusion study. Table 3-2 lists the performance specifications of the four different low-cost IMU are used in this study.

Table 3-1: Low-Cost IMU performance specifications [75; 76; 77].

\begin{tabular}{|c|c|c|c|c|}
\hline Performance Specification & ADIS-16405 & $\begin{array}{c}\text { Crossbow VG400CA- } \\
200\end{array}$ & $\begin{array}{c}\text { Crossbow } \\
\text { DMU(VG400)-100 }\end{array}$ & $\begin{array}{c}\text { Crossbow } \\
\text { IMU400CC-200 }\end{array}$ \\
\hline SUAV & RetroFit Blue YF-22 & Green YF-22 & Red YF-22 & Blue YF-22 \\
\hline \multicolumn{5}{|c|}{ Rate Gyroscopes } \\
\hline Dynamic Range (deg/sec) & \pm 150 & \pm 200 & \pm 100 & \pm 200 \\
\hline Resolution (deg/sec) & 0.025 & 0.05 & 0.05 & 0.05 \\
\hline Nonlinearity (\%FS Range) & 0.1 & 0.3 & 0.3 & 0.3 \\
\hline Bias Stability (deg/hr) & 25.2 & Not Reported & Not Reported & Not Reported \\
\hline $1-\sigma$ Initial Bias Error $(\mathrm{deg} / \mathrm{s})$ & \pm 3 & Not Reported & Not Reported & Not Reported \\
\hline Angular Random Walk (deg/ $\sqrt{ }(\mathrm{hr}))$ & 2 & 1.7 & 0.85 & 4.5 \\
\hline \multicolumn{5}{|c|}{ Accelerometers } \\
\hline Dynamic Range $(\boldsymbol{g})$ & \pm 18 & \pm 10 & \pm 8.5 & \pm 10 \\
\hline Resolution (mg ) & 3.33 & 1.25 & 0.25 & 1.25 \\
\hline Nonlinearity (\%FS Range) & 0.1 & 1 & 1 & 1 \\
\hline Bias Stability $((\mathrm{m} / \mathrm{s}) / \mathrm{hr})$ & 70.61 & Not Reported & Not Reported & Not Reported \\
\hline $1-\sigma$ Initial Bias Error (mg) & \pm 50 & Not Reported & Not Reported & Not Reported \\
\hline Velocity Random Walk $((\mathrm{m} / \mathrm{s}) / \sqrt{ }(\mathrm{hr}))$ & 0.2 & 0.5 & 0.1 & 0.5 \\
\hline
\end{tabular}

As Table 3-2 indicates, the performance specifications of the four MEMS IMU are slightly different. That is in simple terms of rand and resolution, and in terms of reported noise characteristics. The Angle Random Walk and Velocity Random Walk specifications are related to the Allan variance curve, and the expected magnitude of white noise in the sensor output [4].

The GPS receiver employed in this study is Novatel's OEM4 single frequency receiver [78]. The static accuracy of the position measurements are reported to be $1.8 \mathrm{~m}$ Circular-ErrorProbable, and the velocity accuracy is reported as $0.03 \mathrm{~m} / \mathrm{s} \mathrm{rms.}$

The last remaining sensor fusion source used in this study is the tri-axis magnetometers that are in the ADIS-16405 IMU of the Gen- $V$ system. The range of the magnetometers is \pm 3.5 gauss with a resolution of $0.5 \mathrm{mGauss}$. The noise density is reported as $0.066 \mathrm{mGauss} / \sqrt{ } \mathrm{Hz}$ [77]. 


\subsection{Flight Testing}

To acquire some the flight data used this study, and to support other projects, flight testing activities were carried out at WVU's Jackson's Mill flight testing facility, located in Jane Lew, West Virginia. Figure 3.10 is a Google Earth picture of the Jackson's Mill facility in which a GPS track of a WVU SUAV flight test is overlaid on the image.

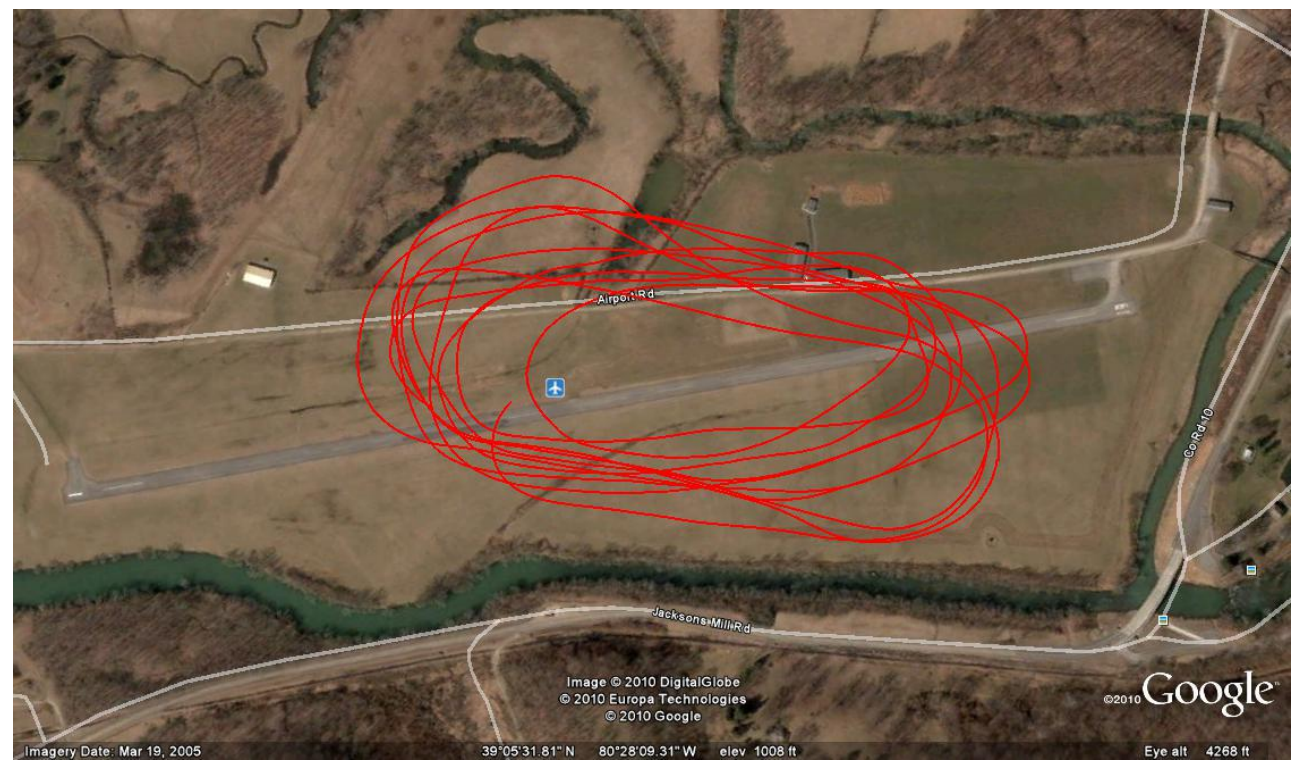

Figure 3.9: GPS Track on Google Earth $®$ Image Jackson's Mill Facility

In order to transport the research aircraft to Jackson's Mill and back, a trailer is outfitted to haul the planes and necessary equipment, as shown in Figure 3.11.
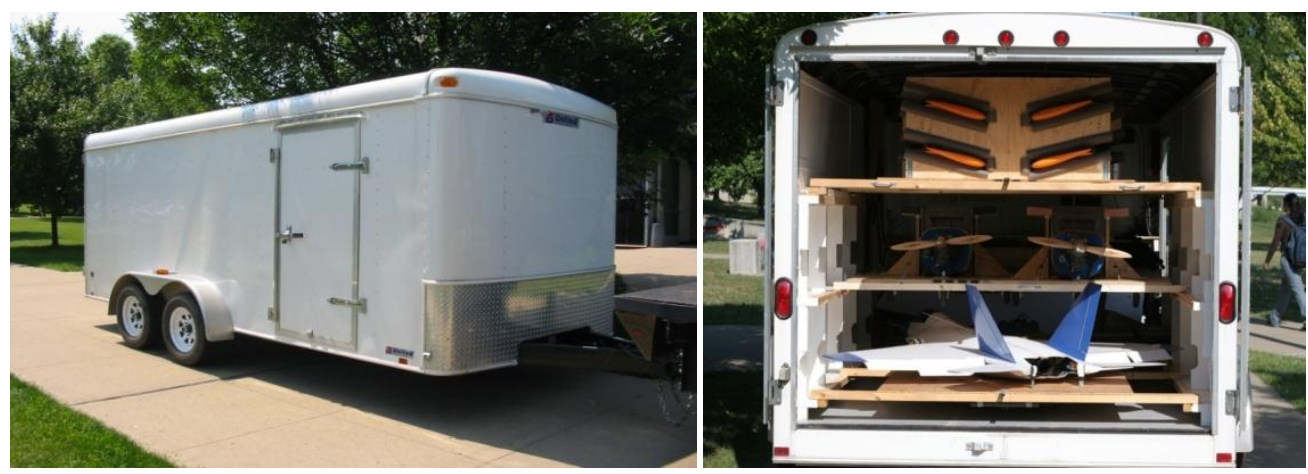

Figure 3.10: WVU Flight Testing Trailer 
Flight testing activities are typically were conducted from March throughout November each year. A full team of graduate students, post-doctoral fellows, and Research Assistant Professors were involved for operational support and guidance.

Additionally, much of the flight data used for this study was collected during the WVU Formation Flight project, and the author of this dissertation is grateful to the many students and faculty who have supported WVU's flight testing activities over the past decade. 


\section{Chapter 4 : Project Objectives}

Within this dissertation project, the overall goal was to design a fault-tolerant attitude estimation algorithm that provides an accurate solution in comparison to a high-quality measurement while only using low-cost components. However, in order to get to that point, key intermediate steps were taken. First, an experimental evaluation of different nonlinear KFs applied to loosely coupled GPS/INS sensor fusion for the purpose of SUAV attitude estimation was conducted. Second, in order to achieve the first step toward fault-tolerance, the GPS/INS sensor fusion algorithms were enhanced to include on-line bias tracking of IMU sensor biases. Third, magnetometers were included as an additional low-cost attitude information source for use in the sensor fusion algorithms. Finally, with the insights obtained along the way, a faulttolerant attitude estimation algorithm was implemented and tested using flight data.

\section{Phase \#1- Fused Attitude Estimation:}

This phase of the research project was the foundation of the study and consists of the design, implementation and comparison of nonlinear estimation algorithms applied to sensor fusion based aircraft navigation using actual flight data. Throughout this process, sensor fusion based navigation algorithms using EKF and SPKF were extensively compared in terms of estimation performance across a variety of formulations. Throughout this comparison, the fused information was GPS measurements loosely coupled to a navigation solution based on low-cost MEMS IMU information (e.g. triad of aircraft body axis angular rates, and specific force measurement). During this comparison, a high-quality mechanical gyroscope (Goodrich ${ }^{\circledR}$ VG34) was available to use as an independent attitude reference. Along with the numerical 
performance metrics, an aim of the stage of the study was to gain the insights into the different nonlinear KFs.

\section{Phase \#2- Calibration with Sensor Fusion}

This phase of the project consisted of including and evaluating navigation system calibration procedures that rely on sensor fusion. The specific aim of this stage was to demonstrate estimation performance increases attributed to sensor fusion based calibration. In addition, during this phase, magnetometers were included into the sensor fusion formulations. At first, the magnetometers seemed useless for attitude estimation without calibration, therefore, a calibration procedure that uses a GPS/INS attitude solution from flight data to determine a calibration model of magnetometers was used. Additionally, the performance of this off-line calibration model was enhanced with on-line calibration.

\section{$\underline{\text { Phase \#3- Fault-Tolerant Attitude Estimation }}$}

Using the insight from phases one and two, this stage of the study consisted of Failure Detection and Identification and Accommodation (FDIA) of sensor failures within the sensor fusion attitude determination system. By improving component level accuracy through sensor fusion based calibration, and exploiting the availability of partially redundant information, the accuracy of the fused system performance is demonstrated to be tolerant of individual components failures. 


\section{Chapter 5 : Nonlinear Estimation}

\subsection{Discrete-Time Kalman Filter}

Before discussing nonlinear estimation, it is appropriate to first discuss the Kalman Filter $(\mathrm{KF})$, which is the optimal state estimator for linear systems that are perturbed with zero-mean and white process and measurement noise that are not correlated with one another $[18 ; 23]$. Specifically, the KF provides state estimate that "minimizes the expected value of the weighted squared estimation error, where the weights are user-defined" [23]. Because the problem considered in this study is discrete, the discrete-time form of the KF is described, and the acronym $\mathrm{KF}$ are used to refer to the discrete-time form, however there is also a continuous form of the Kalman Filter, which is known as the Kalman-Bucy Filter [18].

The KF consists of two sequential procedures, the prediction and measurement-update; the associated well-known equations are outlined in numerous textbooks $[18 ; 24 ; 37 ; 25 ; 23]$. The KF not only provides the estimate of the desired state, but also an error covariance matrix that represents the filter's uncertainty of the estimated state vector. The prediction step of the filter relies on a mathematical model and the 'a priori' estimate of the state vector and error covariance matrix, and possibly an external input to propagate an estimate of the state the next discrete time-step. The measurement-update procedure relies on an external measurement that is related to the state vector through some known observation function, and is used to suppress error growth of the state estimate.

Between the two sequential procedures of the KF (prediction and measurement-update), assumptions are made that quantify uncertainties. Namely, a process-noise covariance matrix, $Q$, 
is assigned by the filter designer to represent the uncertainty of the model, and a measurementnoise covariance matrix, $R$, is assigned by the designer to represent the uncertainty associated with the external measurement and observation function used during the measurement-update procedure. As an example, if the scalar case is considered, assigning the extreme value of 0 for process-noise, $Q$, represents assuming a perfect prediction from the model, whereas 0 for the measurement covariance, $R$, represents assuming a perfect measurement. The relative magnitude between $Q$ and $R$ represent the weighting between prediction and measurement update. Assigning the $Q$ and $R$ matrices for best performance is known as "filter tuning".

First, to begin the algorithm, either a known initial condition or an assumption of the initial condition of the state vector, $\mathbf{x}_{0}$, and state error covariance, $P_{0}$, must be assigned. In particular, the state error covariance matrix is initialized according to the assumed uncertainty of the initial condition assigned to the state vector. That is, if the initial condition is precisely known, the initialized error covariance should be small in magnitude, and if there exists a lot uncertainty of the initial condition, then a large magnitude initial error-covariance matrix should be assigned [23], or more formally

$$
P_{0}=E\left[\left(\hat{\mathbf{x}}_{0}-\mathbf{x}\right)\left(\hat{\mathbf{x}}_{0}-\mathbf{x}\right)^{T}\right]
$$

Next, over a discrete time-step, the state mean and error covariance are predicted using a linear dynamic model, $F$, and the 'a priori' information of the state vector and error covariance from the previous discrete time-step. 


$$
\begin{aligned}
& \hat{\mathbf{x}}_{k \mid k-1}=F_{k \mid k-1} \hat{\mathbf{x}}_{k-1 \mid k-1}+w_{k} \\
& P_{k \mid k-1}=F_{k-1 \mid k-1} P_{k \mid k-1} F_{k-1 \mid k-1}^{T}+Q_{k-1}
\end{aligned}
$$

Where, in this case, zero-mean, white process-noise, $w_{k}$, is assumed to be additive noise that is uncorrelated with the measurement noise. Note that in Equations (5.2) and (5.3) the discrete subscripts are used to distinguish the step of the filter relative to prediction and update. That is $\hat{\mathbf{x}}_{k \mid k-1}$ is interpreted as the estimate at time $k$, given the ' $a$ priori' information from $k-1$, and immediately refers to the state vector after the prediction, but before being updated by the Kalman gain matrix. This is relatively common filtering subscripting convention, which is used throughout this dissertation. For the prediction procedure to be written in a more generic form, Equation (5.2), may also include an input to the linear system, provided some known linear input relationship, $G$, as noted by Simon in his textbook [23].

$$
\hat{\mathbf{x}}_{k \mid k-1}=F_{k \mid k-1} \hat{\mathbf{x}}_{k-1 \mid k-1}+G_{k \mid k-1} \mathbf{u}_{k-1 \mid k-1}+w_{k}
$$

During the update procedure, the predicted state vector, $\hat{\mathbf{x}}_{k \mid k-1}$, is used with the linear observation function, $\boldsymbol{H}$, to provide the predicted system outputs.

$$
\mathbf{y}_{k \mid k-1}=H_{k-1 \mid k-1} \hat{\mathbf{x}}_{k \mid k-1}+v_{k}
$$

Next, the Kalman gain matrix is calculated by using the predicted error covariance, observation function, and assumed measurement-noise covariance, $R$. 


$$
K_{k}=P_{k \mid k-1} H_{k \mid k-1}^{T}\left(H_{k \mid k-1} P_{k \mid k-1} H_{k \mid k-1}^{T}+R_{k}\right)^{-1}
$$

The measurement-noise covariance values also assume zero-mean additive, and white noise that is uncorrelated with the process-noise. Finally, the Kalman gain matrix is used to update both the mean and error covariance of the states.

$$
\begin{aligned}
& \hat{\mathbf{x}}_{k \mid k}=\hat{\mathbf{x}}_{k \mid k-1}+K_{k}\left(\mathbf{z}_{k}-\mathbf{y}_{k \mid k-1}\right) \\
& P_{k \mid k}=\left(I-K_{k} H_{k \mid k-1}\right) P_{k \mid k-1}
\end{aligned}
$$

After being updated with the Kalman gain, the state vector is referred to the 'a posteriori' state vector. In Equation (5.7), the difference between the predicted output, $\mathbf{y}$, and the measurement $\mathbf{z}_{k}$ is referred to as the 'innovation residual', and represents the additional information that the external measurement provides [23].

The nonlinear estimators employed in this study are realizations of the KF for nonlinear systems and employ the same predictor-corrector framework. In fact, nonlinear forms of the KF

are approximations of the $\mathrm{KF}$ based on linearization. However, due to nonlinearity, the optimality proof of the KF is lost.

\subsection{Extended Kalman Filter}

In general, several possible uncertainty assumptions are possible for both process-noise and measurement-noise. The traditional approach is to assume additive-noise; however a more 
general derivation of the EKF is available [23]. Utilizing both additive and non-additive noise assumptions is relevant to sensor fusion for SUAV navigation therefore; both forms are discussed in this dissertation.

\subsubsection{With Additive Noise}

Next, the well-known discrete-time EKF equations are shown [25; 24; 18]. The EKF provides estimates for both the state mean and error-covariance using predictor Equations (5.9-10) corrector Equations (5.11-12) architecture. First, just as with the linear KF, the initial state vector and error covariance matrix must be initialized. For the EKF, the prediction function, $f$, is nonlinear,

$$
\begin{gathered}
\hat{\mathbf{x}}_{k \mid k-1}=f\left(\hat{\mathbf{x}}_{k-1 \mid k-1}, \mathbf{u}_{k}\right)+w_{k} \\
P_{k \mid k-1}=A_{\mathbf{x}, k} P_{k-1 \mid k-1} A_{\mathbf{x}, k}^{T}+Q_{k-1}
\end{gathered}
$$

and the state vector is predicted using the in-tact nonlinear functions, as shown in Equation (5.9), however the error-covariance requires a linearized approximation of the model, $A$, as shown in Equation (5.10). Therefore, a Jacobian matrix is calculated that is composed of first order Taylor series expansions of each of the nonlinear state-transition functions with respect to the states at each discrete time step. 


$$
A_{\mathbf{x}, k}=\left[\begin{array}{ccc}
\frac{\partial f\left(\mathbf{x}_{1}\right)}{\delta \mathbf{x}_{1}} & \ldots & \frac{\partial f\left(\mathbf{x}_{1}\right)}{\delta \mathbf{x}_{n}} \\
\vdots & \ddots & \vdots \\
\frac{\partial f\left(\mathbf{x}_{n}\right)}{\delta \mathbf{x}_{1}} & \ldots & \frac{\partial f\left(\mathbf{x}_{n}\right)}{\delta \mathbf{x}_{n}}
\end{array}\right]
$$

During the measurement-update procedure, the predicted states are used in the nonlinear observation function, $\boldsymbol{h}$, to provide the predicted output vector, $\mathbf{y}$.

$$
\mathbf{y}_{k \mid k-1}=h\left(\hat{\mathbf{x}}_{k \mid k-1}, \mathbf{c}_{k}\right)+v_{k}
$$

Just, as the prediction step, a linearization of the nonlinear observation function, $\mathrm{h}$, is required, Equation (5.12) as denoted by $H_{\mathbf{x}}$.

$$
K_{k}=P_{k \mid k-1} H_{\mathbf{x}, k}^{T}\left(H_{\mathbf{x}, k} P_{k \mid k-1} H_{\mathbf{x}, k}^{T}+R_{k}\right)^{-1}
$$

As shown in Equation (5.13), using the linearized observation function, a Kalman gain is calculated using the predicted error-covariance and measurement-noise covariance assumptions, $R$. Where, just as the KF, the Kalman gain matrix is derived based on a cost function that minimizes the mean-square of the expected estimation error [24], but due to linearization it is an approximation. This is equivalent to reducing the trace of the predicted error covariance [23]. 


$$
\begin{aligned}
& \hat{\mathbf{x}}_{k \mid k}=\hat{\mathbf{x}}_{k \mid k-1}+K_{k}\left(\mathbf{z}_{k}-h\left(\hat{\mathbf{x}}_{k \mid k-1}, \mathbf{c}_{k}\right)\right) \\
& P_{k \mid k}=\left(I-K_{k} H_{k \mid k-1}\right) P_{k \mid k-1}
\end{aligned}
$$

Using the Kalman gain, the estimate of the state vector is updated using the innovation residual, and the error covariance matrix is updated for the next discrete time step.

\subsubsection{With Non-Additive Noise}

It is also possible to formulate the EKF more general with respect to noise assumptions. Specifically, it is not necessarily correct to assume additive process and measurement noise, and they may be considered an input to the state transition function [23].

$$
\hat{\mathbf{x}}_{k \mid k-1}=f\left(\hat{\mathbf{x}}_{k-1 \mid k-1}, \mathbf{u}_{k}, w_{k}\right)
$$

In this case, the error-covariance is propagated, not only with a linearization of the states, $A_{\mathbf{x}, k}$, but also with respect to the process-noise, $A_{w, k}$

$$
A_{w, k}=\left[\begin{array}{ccc}
\frac{\partial f\left(\mathbf{x}_{1}\right)}{\delta w_{1}} & \ldots & \frac{\partial f\left(\mathbf{x}_{1}\right)}{\delta w_{n}} \\
\vdots & \ddots & \vdots \\
\frac{\partial f\left(\mathbf{x}_{n}\right)}{\delta w_{1}} & \ldots & \frac{\partial f\left(\mathbf{x}_{n}\right)}{\delta w_{n}}
\end{array}\right]
$$

such that the error-covariance prediction equation is defined as shown in Equation (5.18).

$$
P_{k \mid k-1}=A_{\mathbf{x}, k} P_{k-1 \mid k-1} A_{\mathbf{x}, k}^{T}+A_{w, k} Q_{k-1} A_{w, k}^{T}
$$


For the non-additive measurement-noise, the observation equation becomes

$$
\mathbf{y}_{k \mid k-1}=h\left(\hat{\mathbf{x}}_{k \mid k-1}, \mathbf{c}_{k}, v_{k}\right)
$$

which in the non-additive measurement noise case, is linearized both about the states, $H_{\mathbf{x}, k}$, and the measurement noise, $H_{w, k}$. Therefore, the Kalman gain equation is updated to include the linearization about the measurement noise.

$$
K_{k}=P_{k \mid k-1} H_{\mathbf{x}, k}^{T}\left(H_{\mathbf{x}, k} P_{k \mid k-1} H_{\mathbf{x}, k}^{T}+H_{v, k} R_{k} H_{v, k}^{T}\right)^{-1}
$$

The Kalman gain is used in the same manner as Equations (5.14-15) to update the state and error-covariance matrices.

\subsection{Sigma-Point Kalman Filter}

Just as the EKF, utilizing noise assumptions that are both additive and non-additive are relevant to sensor fusion navigation, and therefore both forms are discussed. The formulation of the SPKF which is described here is available in textbooks [24;23] and was originally developed by Julier and Uhlmann $[19 ; 30]$. 


\subsubsection{With Additive Noise}

For the SPKF, first, the state vector and error-covariance matrix must be initialized, just as the KF and EKF. During the prediction portion of the SPKF, when considering the case of additivenoise, sigma-points are deterministically selected by using the previous time-step 'a posteriori' mean and error covariance. A popular variant to the SPKF is the Unscented Kalman Filter (UKF), which uses the Unscented Transformation, [19; 30].



where $\chi$ represents the sigma points and $P_{k-1 \mid k-1}$ is 'a posterior' the error covariance matrix. Note that, $\gamma$ is a parameter that determines the spread of the sigma-points; van der Merwe and Wan [31; 33] and Julier and Uhlmann [30; 19] provide guidance for selecting this parameter, and the assignment of this value discussed later in this section, so that the conceptually equivalent prediction and measurement-update procedures to the KF and EKF can be discussed first. During the state prediction procedure, each set of sigma-points are propagated through the nonlinear equations, $f$, with the option of an input vector $\mathbf{u}_{k .}$

$$
\chi_{k \mid k-1}=f\left(\chi_{k-1 \mid k-1}, \mathbf{u}_{k}\right)+w_{k}
$$

The predicted state vector is then numerically calculated as a weighted average of the sigmapoints that have been propagated in time with the nonlinear the state-transition function, $f$. 


$$
\hat{\mathbf{x}}_{k \mid k-1}=\sum_{i=0}^{2 l} w_{i}^{m} \chi_{k \mid k-1}^{i}
$$

Again, the criteria for assigning the weights as offered in van der Merwe, Wan and Julier [31], and are discussed later in this section. The predicted error covariance matrix is also calculated as a weighted variance of the sigma-points

$$
P_{k \mid k-1}=\sum_{i=0}^{2 l} w_{i}^{C}\left(\chi_{k \mid k-1}^{i}-\hat{\mathbf{x}}_{k \mid k-1}\right)\left(\chi_{k \mid k-1}^{i}-\hat{\mathbf{x}}_{k \mid k-1}\right)^{T}+Q_{k-1}
$$

where the process-noise, $Q$, is assumed additive. This concludes the section of the algorithm that is conceptually equivalent to the EKF and KF prediction step.

During the measurement-update procedure, first, using the predicted state vector, $\hat{\mathbf{x}}_{k \mid k-1}$, and predicted state error-covariance matrix, $P_{k \mid k-1}$, sigma-points are again generated using the Unscented Transformation $[19 ; 30]$.

$$
\chi_{k \mid k-1}=\left[\begin{array}{lll}
\hat{\mathbf{x}}_{k \mid k-1} & \hat{\mathbf{x}}_{k \mid k-1}+\gamma \sqrt{P_{k \mid k-1}} & \hat{\mathbf{x}}_{k \mid k-1}-\gamma \sqrt{P_{k \mid k-1}}
\end{array}\right]
$$

Output sigma-points are then calculated using the nonlinear observation equations, $h$, with the option of an input $\mathbf{c}_{k}$, and assuming additive process noise. 


$$
Y_{k \mid k-1}=h\left(\chi_{k \mid k-1}, \mathbf{c}_{k}\right)+v_{k}
$$

Next, the predictions of the output vector, $\mathbf{y}$, is calculated as a weighted sum.

$$
\mathbf{y}_{k \mid k-1}=\sum_{i=0}^{2 l} w_{i}^{m} Y_{k \mid k-1}^{i}
$$

Similar to the predicted state error covariance matrix, from Equation (5.20), the output errorcovariance matrix is calculated by comparing the mean of the output with the output sigmapoints.

$$
P_{y_{k} y_{k}}=\sum_{i=0}^{2 l} w_{i}^{C}\left(Y_{k \mid k-1}^{i}-\mathbf{y}_{k \mid k-1}\right)\left(Y_{k \mid k-1}^{i}-\mathbf{y}_{k \mid k-1}\right)^{T}+R_{k}
$$

In addition, the cross-covariance matrix of the predicted states and the observed outputs is calculated through a weighted covariance.

$$
P_{\mathbf{x}_{k} \mathbf{y}_{k}}=\sum_{i=0}^{2 l} w_{i}^{C}\left(\chi_{k \mid k-1}^{i}-\hat{\mathbf{x}}_{k \mid k-1}\right)\left(Y_{k \mid k-1}^{i}-\hat{\mathbf{y}}_{k \mid k-1}\right)^{T}
$$

Finally, the Kalman gains are calculated, and the both the states and the error-covariance are updated. 


$$
\begin{gathered}
K_{k}=P_{\mathbf{x}_{k} \mathbf{y}_{k}} / P_{\mathbf{y}_{k} \mathbf{y}_{k}} \\
\hat{\mathbf{x}}_{k \mid k}=\hat{\mathbf{x}}_{k \mid k-1}+K_{k}\left(\mathbf{z}_{k}-\mathbf{y}_{k \mid k-1}\right)
\end{gathered}
$$

where, $\mathbf{z}_{\mathrm{k}}$ is the measurement vector.

$$
P_{k \mid k}=P_{k \mid k-1}-K_{k} P_{y_{k} y_{k}} K_{k}^{T}
$$

This procedure is repeated at each discrete time step.

Next, the procedure for defining the weight vectors $\left(w^{m}\right.$ and $\left.w^{c}\right)$ and sigma-point spread parameter , $\gamma$, used in the SPKF algorithm are discussed. van der Merwe and Wan $[33 ; 31]$ suggest the following relationship for $\gamma$ :

$$
\gamma=\sqrt{l+\lambda}
$$

where $l$ refers to the dimension of state vector, while, $\lambda$, is further parameterized with two additional values: $\alpha$ and $\kappa$.

$$
\lambda=\alpha^{2}(l+\kappa)-l
$$

The range suggested for $\alpha$ is between 0.001 and 1 , and it is mentioned that $\kappa$ for most applications is zero. van der Merwe, Wan and Julier [31]emphasize that the best selection of parameters is dependent on the problem. Also, the vectors used for weighted averaging are defined as: 


$$
\begin{gathered}
w_{0}^{m}=\frac{\lambda}{(l+\lambda)} \\
w_{0}^{c}=w_{0}^{m}+\left(1-\alpha^{2}+\beta\right) \\
w_{i}^{c}=w_{i}^{m}=\frac{1}{2(l+\lambda)}
\end{gathered}
$$

Throughout this dissertation the parameters were selected as $\alpha=0.5$ and $\beta=2$. For Gaussian distributions, $\beta=2$ is optimal [19].

\subsubsection{With Non-Additive Noise}

During the prediction portion of the SPKF, in order to consider the generic case of nonadditive process-noise, the state vector is augmented with the process-noise [30]

$$
\hat{\mathbf{x}}_{k-1 \mid k-1}^{a}=\left[\begin{array}{ll}
\hat{\mathbf{x}}_{k-1 \mid k-1} & w_{k}
\end{array}\right], P_{k-1 \mid k-1}^{a}=\left[\begin{array}{cc}
P_{\mathbf{x} k-1 \mid k-1} & 0 \\
0 & Q_{k-1}
\end{array}\right]
$$

such that, sigma-points are deterministically selected by using the previous time-step ' $a$ posteriori' augmented mean and error covariance, with the Unscented Transformation, [30; 19]. 


$$
\chi_{k-1 \mid k-1}=\left[\begin{array}{lll}
\hat{\mathbf{x}}_{k-1 \mid k-1}^{a} & \hat{\mathbf{x}}_{k-1 \mid k-1}^{a}+\gamma \sqrt{P_{k-1 \mid k-1}^{a}} \quad \hat{\mathbf{x}}_{k-1 \mid k-1}^{a}-\gamma \sqrt{P_{k-1 \mid k-1}^{a}}
\end{array}\right]
$$

Next, each set of sigma-points are propagated through the nonlinear equations, $f$, with the option of an input vector $\mathbf{u}_{k} \cdot$.

$$
\chi_{k \mid k-1}=f\left(\chi_{k-1 \mid k-1}, \mathbf{u}_{k}, w_{k}\right)
$$

Note that in this case, the process-noise assumptions, $w_{k}$, are included within the sigma-points, $\chi$ , and may be freely applied during the prediction procedure, such that the resulting system is statistically linearized around that point [34]. This is an advantage over the form which assumes additive noise. The predicted state vector is then calculated as a weighted average of the sigmapoints that have been updated with the state-transition function, $f$.

$$
\hat{\mathbf{x}}_{k \mid k-1}=\sum_{i=0}^{2 l} w_{i}^{m} \chi_{k \mid k-1}^{i}
$$

The predicted error covariance matrix is also calculated as a weighted variance of the sigmapoints. 


$$
P_{\mathbf{x} k \mid k-1}=\sum_{i=0}^{2 l} w_{i}^{C}\left(\chi_{k \mid k-1}^{i}-\hat{\mathbf{x}}_{k \mid k-1}\right)\left(\chi_{k \mid k-1}^{i}-\hat{\mathbf{x}}_{k \mid k-1}\right)^{T}
$$

During the measurement-update procedure, the state vector is now augmented with sigma-points that correspond to the measurement noise assumptions

$$
\hat{\mathbf{x}}_{k \mid k-1}^{a}=\left[\begin{array}{ll}
\hat{\mathbf{x}}_{k \mid k-1} & v_{k}
\end{array}\right], P_{k \mid k-1}^{a}=\left[\begin{array}{cc}
P_{\mathbf{x} k \mid k-1} & 0 \\
0 & R_{k}
\end{array}\right]
$$

where only the portion of the error-covariance matrix with respect to the states is carried over from the prediction step. Then, using the predicted state vector, $\hat{\mathbf{x}}_{k \mid k-1}$, and predicted state errorcovariance matrix that is augmented with the measurement noise, $P_{k \mid k-1}^{a}$, sigma-points are again calculated using the Unscented Transformation [19; 30].

$$
\chi_{k \mid k-1}=\left[\begin{array}{lll}
\hat{\mathbf{x}}_{k \mid k-1}^{a} & \hat{\mathbf{x}}_{k \mid k-1}^{a}+\gamma \sqrt{P_{k \mid k-1}^{a}} & \hat{\mathbf{x}}_{k \mid k-1}^{a}-\gamma \sqrt{P_{k \mid k-1}^{a}}
\end{array}\right]
$$

Output sigma-points are then calculated using the nonlinear observation equations, $h$, with the option of an input $\mathbf{c}_{k}$ 


$$
Y_{k \mid k-1}=h\left(\chi_{k \mid k-1}, \mathbf{c}_{k}, v_{k}\right)
$$

where, again, the measurement noise is not assumed additive. Next, the predictions of the output vector, $\mathbf{y}$, is calculated as a weighted sum.

$$
\mathbf{y}_{k \mid k-1}=\sum_{i=0}^{2 l} w_{i}^{m} Y_{k \mid k-1}^{i}
$$

Similar to the predicted state error-covariance matrix, the output error-covariance matrix is calculated by comparing the mean of the output with the output sigma-points.

$$
P_{\mathbf{y}_{k} \mathbf{y}_{k}}=\sum_{i=0}^{2 l} w_{i}^{C}\left(Y_{k \mid k-1}^{i}-\mathbf{y}_{k \mid k-1}\right)\left(Y_{k \mid k-1}^{i}-\mathbf{y}_{k \mid k-1}\right)^{T}
$$

In addition, the cross-covariance matrix of the predicted states and the observed outputs is calculated through a weighted covariance, however different from the additive case, the processnoise covariance, $Q$, is implicitly considered within the augmented system. 


$$
P_{\mathbf{x}_{k} \mathbf{y}_{k}}=\sum_{i=0}^{2 l} w_{i}^{C}\left(\chi_{k \mid k-1}^{i}-\hat{\mathbf{x}}_{k \mid k-1}\right)\left(Y_{k \mid k-1}^{i}-\hat{\mathbf{y}}_{k \mid k-1}\right)^{T}
$$

Finally, the Kalman gains are calculated, and the both the states and the error-covariance are updated, just as in the additive case.

The rules for assigning, the weights and scaling parameters are the same for the non-additive noise SPKF, however, it should be noted that, $l$, that appears in Equations (5.32-36) refers to the dimension of the augmented system.

\subsection{Information Filter}

While for linear problems, the IF and KF are algebraically equivalent, the IF form has a good property with respect to handling multiple measurement-updates in the form of a summation [20; $21 ; 22]$. Because the overall goal of this study is to develop a fault-tolerant algorithm, the ability to handle multiple measurements updates is crucial; therefore, the nonlinear extensions of the IF are also included in this discussion.

Instead of mean and error-covariance, the information form of the KF propagates the time evolution of the information state vector, $i$, and the Fisher information matrix $I[24 ; 23]$. For the information prediction procedure, a prediction may be carried out in the information space [23], or more conveniently may be carried out in the state-space and then converted to the information space. Therefore for a nonlinear IF, either the prediction step from an EKF or SPKF, using either an additive or not is performed, and the prediction of the Fisher information is calculated as the inverse of the predicted error-covariance matrix. The formulation of the IF for linear systems is available in textbooks [23; 24], and has been extended for use in nonlinear systems $[20 ; 21 ; 22]$. 


$$
I_{k \mid k-1}=P_{k \mid k-1}^{-1}
$$

Next, the prediction of the information state vector is calculated, which related to the predicted error-covariance and predicted state vector, through Equation (5.48).

$$
i_{k \mid k-1}=P_{k \mid k-1}^{-1} x_{k \mid k-1}
$$

As mentioned, the primary difference between the IF and KF arises during measurement-update. Specifically, for the measurement-update procedure, an information matrix and vector is calculated using each available external measurement source $(j=1$ to $N)$, and the update is reduced to a trivial summation of information.

$$
\begin{array}{r}
i_{k \mid k}=i_{k \mid k-1}+\sum_{j=1}^{N} i_{j, k} \\
I_{k \mid k}=I_{k \mid k-1}+\sum_{j=1}^{N} I_{j, k}
\end{array}
$$

where $i_{j, k}$ and $I_{j, k}$ are calculated for each measurement source. The procedure for determining the information of a measurement starts with the nonlinear observation function that is either assumed to have additive noise 


$$
y_{j, k}=h_{j}\left(x_{k \mid k-1}, \mathbf{c}_{j, k}\right)+v_{j, k}
$$

or measurement noise used implicitly used in the function

$$
y_{j, k}=h_{j}\left(x_{k \mid k-1}, \mathbf{c}_{j, k}, v_{j, k}\right)
$$

To handle nonlinearity, the observation function is locally linearized,$H$, using either statistical

linear regression method, as is the case of the Unscented Information Filter (UIF) [21], or by using a Jacobian of the observation functions [20], which is case for the Extended Information Filter (EIF). Because there are some key differences between the EIF and UIF measurement information calculation procedures; therefore, they are discussed in separate sub-sections.

\subsubsection{Extended Information Filter Measurement Information Calculation}

The EIF linearizes each nonlinear observation function $(j=1$ to $N)$, using a Jacobian, and the Fisher information of observation is calculated using the assumed measurement-error covariance matrix [20]

$$
I_{j, k}=H_{j, k}^{T} R_{j, k}^{-1} H_{j, k}
$$

where the predicted information matrix is updated by adding the observation information from each source, by using Equation (5.50). At the point, some authors [24; 23] suggest calculating a Kalman gain and proceeding as the EKF 


$$
K_{k}=\left(I_{k \mid k}\right)^{-1} H_{k}^{T} R_{k}^{-1}
$$

however calculating a Kalman gain, at this point, does not take advantage of handling multiple measurement-update sources, therefore it is useful to calculate the information state vector for each source, using the external measurement.

$$
i_{j, k}=H_{j, k}^{T} R_{j, k}^{-} \mathbf{z}_{j, k}
$$

At this point, the information state vector is updated, $i_{k \mid k}$, by summation as shown in Equation (5.57).

\subsubsection{Unscented Information Filter Measurement Information Calculation}

For the UIF, the statistical linearization, Weighted Least Squared WLSR [21; 34] is used for linearization, just as in the UKF. For this case, the more general non-additive form, is considered where if the system is augmented with the assumed measurement noise, this linearization is described as shown in Equation (5.56),

$$
\mathbf{y}_{j, k} \cong\left[\begin{array}{ll}
H_{\mathbf{x} j, k} & H_{v j, k}
\end{array}\right]\left[\begin{array}{c}
\hat{\mathbf{x}}_{k \mid k-1} \\
v_{j, k}
\end{array}\right]+b_{j, k}
$$


such that the linearization is conducted both about the states and the measurement noise. Within, Equation (5.57), $b_{j, k}$ represents a bias attributed to the statistical linearization. By using the intact nonlinear observation function, this value is determined as the difference between the nonlinear observation and the linearization.

$$
b_{j, k}=h_{j}\left(\hat{\mathbf{x}}_{k \mid k-1}, \mathbf{c}_{j, k}, v_{j, k}\right)-\left(\left[\begin{array}{ll}
H_{\mathbf{x} j, k} & H_{v j, k}
\end{array}\right]\left[\begin{array}{c}
\hat{\mathbf{x}}_{k \mid k-1} \\
v_{j, k}
\end{array}\right]\right)
$$

Using this term, the measurement information matrix $I_{j, k}$ and information vector $i_{j, k}$ for the $j^{\text {th }}$ measurement is calculated using the external measurement [21],

$$
\begin{aligned}
& i_{j, k}=H_{\mathbf{x} j, k}^{T} \bar{R}_{j, k}^{-1}\left(\mathbf{z}_{j, k}-b_{j, k}\right) \\
& I_{j, k}=H_{\mathbf{x} j, k}^{T} \bar{R}_{j, k}^{-1} H_{\mathbf{x} j, k}
\end{aligned}
$$

where $\bar{R}_{j, k}$ is the covariance matrix associated with both the sum of the linearized measurementnoise $R_{j}$ and the noise associated with linearization.

$$
\bar{R}_{j, k}=H z_{j, k} R_{j} H z_{j, k}^{T}+P_{j, e e}=P_{z_{j, k} z_{j, k}}-H x_{j, k} P_{k \mid k-1} H x_{j, k}^{T}
$$

A detailed procedure for deriving Equations (5.55-60) is shown in [21]. At any point, of the filter, a conversion from the information space to the state space can be used to obtain the estimated error-covariance matrix and state vector are calculated respectively. 


$$
\begin{gathered}
P_{k \mid k}=I_{k \mid k}^{-1} \\
\hat{\mathbf{x}}_{k \mid k}=P_{k \mid k} \mathbf{y}_{k \mid k}
\end{gathered}
$$

\subsection{Mixed Kalman Filters}

It has been suggested by Changey et. al. [45] that the prediction and update steps of EKF and SPKF filters can be used interchangeably to form a Mixed Extended/Unscented filter. In this context, if a time-update or measurement procedure is linear, then the standard $\mathrm{KF}$ equations may be interchanged with the EKF or SPKF counterparts. Similarly, if either the state-transition or observation function sets are linear, then a prediction or update procedure from the KF can be combined with either of the nonlinear approaches $[79 ; 80]$. A UKF that uses the normal KF procedure for a linear portion of the filter is referred to as a Roa-Blackwellised UKF by Briers, Maskell and Wright [80] and are compared with a UKF formulation that uses sigma points to assume additive and non-additive noises by Hao et. al. [79] and showed the same estimation error with reduction of computational burden with additive linear noise is considered. 


\section{Chapter 6 : Sensor Fusion Formulations}

\subsection{Coordinate Systems}

Before the different sensor fusion formulations are discussed, it is first appropriate to define the coordinate systems utilized to formulate the problem. A primary coordinate system that is utilized throughout this study to formulate the various sensor fusion navigation algorithms is the conventional aircraft-body axis. In this coordinate system, the positive $\mathrm{x}$-axis is forward in reference to the pilot's cock-pit, the positive y-axis is to the right of the pilot's cock-pit, and the z-axis is positive in the down direction from the pilot's perspective as shown in Figure 6.1.

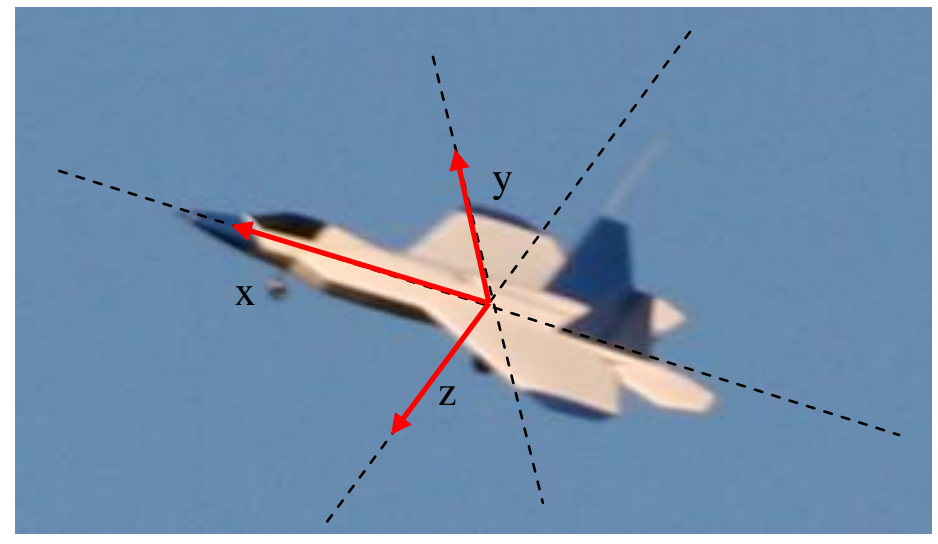

Figure 6.1: Aircraft body-axis convention.

In Figure 6.1, from the pilots perspective, pitch is positive when the nose is rotates upward, roll is positive when the plane banks to the right, and yaw is positive when the plane turns from left to right from the pilots perspective. Each of these rotations follow the convention associated with the right-hand rule.

The other coordinate system utilized in the sensor fusion formulations of this study is a local geodetic navigation frame, of which GPS measurements of position and velocity are used. 
However, the GPS receiver used for this study provided the measurements of position and velocity in an ECEF frame that defined in Figure 5.1.



Figure 6.2: ECEF Coordinate Frame [image reproduced from Novatel [78]]

Where the z-axis is parallel to the poles of the Earth, the x-axis is defined at the intersection of the equator and the WGS-84 defined prime meridian, and the y-axis finishes the right handed coordinate system. However, prior to being used for sensor fusion, these measurements are transformed to a local SWD geodetic frame. To transform from this ECEF frame to the local geodetic frame, three rotations are needed. These rotations are defined according to the latitude ,$\varphi$, and longitude,$\lambda$, coordinates of the location about which the local frame is defined [8].

$$
C_{e}^{g}=\left[\begin{array}{ccc}
-\sin \varphi \cos \lambda & -\sin \varphi \sin \lambda & \cos \varphi \\
\sin \lambda & \cos \lambda & 0 \\
-\cos \varphi \cos \lambda & -\cos \varphi \sin \lambda & -\sin \varphi
\end{array}\right]
$$


By subtracting the origin in the ECEF frame and rotating with the DCM, the GPS solution is transformed into the SWD frame, which is more convenient for local navigation. The local coordinate system is defined on image of WVU's Flight Testing field in Figure 6.3.



Figure 6.3: Local geodetic SWD coordinate system at WVU's Jackson's Mill flight testing facility.

\subsection{GPS/INS Sensor Fusion Navigation Formulations}

In this section of the dissertation, different GPS/INS sensor fusion navigation formulations that employed in the study are presented and discussed. The formulations are written in a manner that is consistent with the predictor-corrector framework that is used by the different nonlinear filtering approaches considered, as discussed in Chapter 5.

The key differences between the various formulations considered include the number of states that the filter estimates, and where in the filter formulation the nonlinear relationships appear. In particular, first an attitude only estimation algorithm is considered, and then a complete Position, Velocity, Attitude (PVA) navigation solution is considered. Changing the 
number of navigation states, in turn, effects where the nonlinear equations are used in the estimation filter; namely, either during the prediction step, the measurement-update procedure, or both. The purpose of varying the formulation was to better evaluate if one form of the nonlinear KF (e.g. EKF or UKF) is better suited for this problem for a particular formulation.

Throughout the description of the sensor fusion formulations, noise-assumptions are not included. This is due to the fact that both additive and non-additive noises are considered in this dissertation. Therefore, initially, all of the formulations are discussed in terms of the state vector, $\mathbf{x}$, measurement vector, $\mathbf{z}$, and prediction and observation functions, $f$ and $h$, with their respective inputs $\mathbf{u}$ and c. Subsequently, another subsection discusses the how the noise terms, $w$ and $v$, are used in the formulations.

\subsubsection{3-State Attitude Estimation}

First, a 3-state attitude estimation formulation is developed, where the three aircraft bodyaxis Euler angles, roll $(\phi)$, pitch $(\theta)$, and yaw $(\psi)$ are estimated $\mathbf{x}=\left[\begin{array}{lll}\phi & \theta & \psi\end{array}\right]^{T}$. During the attitude prediction procedure, measured angular rates in the body-axis, roll rate $(p)$, pitch rate $(q)$

, and yaw rate I of the aircraft are provided by an IMU: $\mathbf{u}=\left[\begin{array}{lll}p & q & r\end{array}\right]_{B}^{T}$, and utilized as inputs to the attitude kinematic equations listed in Equations (6.(2-4)) [81]: 


$$
\begin{gathered}
\dot{\phi}=p+q \sin \phi \tan \theta+r \cos \phi \tan \theta \\
\dot{\theta}=q \cos \phi-r \sin \phi \\
\dot{\psi}=(q \sin \phi+r \cos \phi) \sec \theta
\end{gathered}
$$

such that the three attitude kinematic equations comprise the nonlinear state-transition function, $f$.

Next, during the measurement-update procedure, the information from the IMU accelerometers is compared with GPS velocity measurements. Using this information, the error growth of the INS attitude states is implicitly regulated through the Direction Cosine Matrix (DCM). The DCM between the aircraft-body axis and the local navigation frame, is a function of the predicted attitude states, and in reference to the nonlinear KF is used as the observation function, $h$.

First, the triad of body-axis specific-force measurements provided by the IMU accelerometers: $\mathbf{c}=\left[\begin{array}{lll}a_{x} & a_{y} & a_{z}\end{array}\right]_{B}^{T}$ are transformed to the local Cartesian navigation frame $(L)$ [82].

$$
\left[\begin{array}{c}
\dot{V}_{x} \\
\dot{V}_{y} \\
\dot{V}_{z}+g
\end{array}\right]_{L}=\left[\begin{array}{ccc}
\cos \psi \cos \theta & -\sin \psi \cos \phi+\cos \psi \sin \theta \sin \phi & \sin \psi \sin \phi+\cos \psi \sin \theta \cos \phi \\
\sin \psi \cos \theta & \cos \psi \cos \phi+\sin \psi \sin \theta \sin \phi & -\cos \psi \sin \phi+\sin \psi \sin \theta \cos \phi \\
-\sin \theta & \cos \theta \sin \phi & \cos \theta \cos \phi
\end{array}\right]\left[\begin{array}{l}
a_{x} \\
a_{y} \\
a_{z}
\end{array}\right]_{B} 6.5
$$


Finally, the outputs of the measurement-update equations, $y$, is compared with a change in GPS velocity in the local frame that occurred since the previous update procedure: $\mathbf{z}=\left[\begin{array}{lll}a_{x}^{G P S} & a_{y}^{G P S} & a_{z}^{G P S}\end{array}\right]_{L}^{T}$. For use within the EKF, a Jacobian of the state-transition equations is required, Equations (6-(2.4)), and the observation function, Equation (6.5), these are included in

\section{Appendix A.}

A more thorough description of the mechanism used to correct the predicted attitude states is that attitude error growth is regulated by referencing the Earth's gravity vector. To be exact, the tri-axial accelerometer's of the IMU measure specific-force along the aircraft body-axes. These three specific-force measurements include the inertial accelerations of the aircraft along the three body-axes, and projections of the acceleration due to gravity along the three aircraft body-axes. On the other hand, changes in GPS velocity (e.g. GPS derived acceleration) provide an indication of the aircraft's inertial acceleration only. However, GPS information is measured relative to the local navigation frame. Therefore, by the accelerometer's measurement of specific force from the aircraft body-axis to the local navigation frame, the inertial acceleration of the aircraft as measured both by GPS and the accelerometers cancel one another in Equation 5.4, and the Earth's gravity vector can be referenced. This is conceptually equivalent to using a set of accelerometers as an inclinometer for static applications [83].

\subsubsection{9-State Position, Velocity, and Attitude Estimation}

A 9-state sensor fusion formulation was also considered, in which the navigation state vector consists of the triad of Cartesian position and velocity within a local frame $(L)$, as well as the roll, pitch, and yaw angles with respect to the aircraft body-axis respectively: 
$\mathbf{x}=\left[\begin{array}{lllllllll}x & y & z & V_{x} & V_{y} & V_{z} & \phi & \theta & \psi\end{array}\right]^{T}$. This particular 9-state algorithm was implemented using and EKF by a previous WVU researcher, Jarrell, for his master's thesis [84; 85], and was adapted for use in this study. This algorithm provides a full PVA solution, where the time-update procedure for the Euler angles is provided by Equations (6-(2.4)). However, different from the 3state formulation, the velocity kinematic equations, Equation (6.4), that utilize the IMU's bodyaxis specific force measurements are included in the prediction step: $\mathbf{u}=\left[\begin{array}{llllll}a_{x} & a_{y} & a_{z} & p & q & r\end{array}\right]_{B}^{T}$. Additionally, the time integration of velocity for position estimates is included in the prediction step of this formulation. Because the velocity states are in the local geodetic coordinate frame, this procedure is a simple one-step time integration of velocity. Upon arranging the nine navigation-states into PVA, the continuous-time update equations, are listed as shown in Equation (6.6).

$$
\dot{\mathbf{x}}=\left[\begin{array}{c}
\dot{x} \\
\dot{y} \\
\dot{z} \\
\dot{V}_{x} \\
\dot{V}_{y} \\
\dot{V}_{z} \\
\dot{\phi} \\
\dot{\theta} \\
\dot{\psi}
\end{array}\right]=\left[\begin{array}{c}
V_{x} \\
V_{y} \\
V_{z} \\
\cos \psi \cos \theta a_{x}+(-\sin \psi \cos \phi+\cos \psi \sin \theta \sin \phi) a_{y}+(\sin \psi \sin \phi+\cos \psi \sin \theta \cos \phi) a_{z} \\
\sin \psi \cos \theta a_{x}+(\cos \psi \cos \phi+\sin \psi \sin \theta \sin \phi) a_{y}+(-\cos \psi \sin \phi+\sin \psi \sin \theta \cos \phi) a_{z} \\
-\sin \theta a_{x}+\cos \theta \sin \phi a_{y}+\cos \theta \cos \phi a_{z}-g \\
p+q \sin \phi \tan \theta+r \cos \phi \tan \theta \\
q \cos \phi-r \sin \phi \\
(q \sin \phi+r \cos \phi) \sec \theta
\end{array}\right]
$$

In the 9-state formulation, the measurement-update procedure uses the entire GPS measurement vector as the external measurement, which consists of both the Cartesian position and velocity 
measured in a local geodetic frame $(L): \mathbf{z}=\left[\begin{array}{llllll}x & y & z & V_{x} & V_{y} & V_{z}\end{array}\right]_{L}^{T}$. Therefore, because the first six predicted states are directly measured by GPS, the observation function is linear,

$$
H=\left[I_{6 \times 6}, O_{6 \times 3}\right]
$$

and the filtering methods regulate the error growth of the attitude states implicitly through the coupled relationships between the velocity and attitude. For use in the EKF, a Jacobian of the prediction function, $f$, is required. This is included within Appendix A of this dissertation.

It is interesting to note a distinct difference in the manner that the sensor information is used in the 3-state and 9-state formulations. Specifically, with respect to the prediction-step in the 3state formulation, the DCM that rotates the IMU specific force measurement vector from the body-axis to the local navigation frame is populated with the Euler angles that are predicted using the latest rate gyroscopes measurements. This is not the case for the 9-state formulation. The 9-state formulation instead uses the Euler angles that are the 'a posteriori' estimates from the previous time-step to create a DCM that rotates the accelerometer information to the local frame. Additionally, the 3-state formulation uses nonlinear relationships both during prediction. Equations (6.(2-4)), and measurement-update, Equations (6.5). On the contrary, the 9-state formulation uses a linear observation function, Equation (6.6).

\subsubsection{Acceleration Vector Attitude Estimation}

Another attitude estimation formulation that relies only on the use of GPS velocity measurements and tri-axial accelerometers readings and does not require the use of a nonlinear estimation filter was considered in this study to provide an additional solution that acts as a worst case performance baseline. The Acceleration Vector Attitude Estimation (AVAE) algorithm was 
developed for direct attitude estimation following the dynamic tilt sensor concept, and is similar to approach outlined by Kingston and Beard [86] which algebraically breaks down the DCM between the aircraft body-axis and the local coordinate system. The contribution of algebraic reduction of the DCM, and the AVAE presented in this section of the dissertation is attributed to Matt Rhudy, and is included within a submission by Gross, Gu, Rhudy, Gururajan, and Napolitano [87].

Instead of using a nonlinear KF, as in the sensor fusion formulations described above, the AVAE algorithm features GPS acceleration in a local frame $(L)$, estimated by numerical differentiation of GPS velocity measurements, and accelerometer measurements obtained in the aircraft body-axis $(B)$. The aircraft Euler angles, roll $(\phi)$, pitch $(\theta)$, and yaw $(\psi)$, define a Direction Cosine Matrix (DCM) between the two coordinate frames [82].

$$
\left[\begin{array}{l}
a_{x} \\
a_{y} \\
a_{z}
\end{array}\right]_{(B)}=\left[\begin{array}{ccc}
1 & 0 & 0 \\
0 & \cos \phi & \sin \phi \\
0 & -\sin \phi & \cos \phi
\end{array}\right]\left[\begin{array}{ccc}
\cos \theta & 0 & -\sin \theta \\
0 & 1 & 0 \\
\sin \theta & 0 & \cos \theta
\end{array}\right]\left[\begin{array}{ccc}
\cos \psi & \sin \psi & 0 \\
-\sin \psi & \cos \psi & 0 \\
0 & 0 & 1
\end{array}\right]\left[\begin{array}{c}
a_{x} \\
a_{y} \\
a_{z}-g
\end{array}\right]_{L}
$$

With measurements from both GPS and accelerometers, the projection of the local gravity vector on the three aircraft body-axes in terms of the three Euler angles are resolved and solved for attitude.

First, an estimate of the aircraft heading angle $(\psi)$ is directly obtained by calculating the instantaneous arctangent to the aircraft trajectory using GPS velocity measurements in the $x$ and $y$ axes within the $(L)$ coordinate frame: 


$$
\psi=\tan ^{-1}\left(\frac{V_{y}}{V_{x}}\right)_{L}
$$

Next, sequential Euler rotations are used to approximate pitch and roll. Where, the rotation through the heading angle defines an intermediate acceleration vector that is denoted with $\operatorname{subscript}(\psi)$ in Equation (6.10)

$$
\left[\begin{array}{c}
a_{x} \\
a_{y} \\
a_{z}
\end{array}\right]_{(\psi)}=\left[\begin{array}{c}
a_{x(L)} \cos \psi+a_{y(L)} \sin \psi \\
a_{y(L)} \cos \psi-a_{x(L)} \sin \psi \\
a_{z(L)}-g
\end{array}\right]
$$

At this point, only the pitch $(\theta)$ and roll $(\phi)$ rotations of the original DCM remain.

$$
\left[\begin{array}{l}
a_{x} \\
a_{y} \\
a_{z}
\end{array}\right]_{(B)}=\left[\begin{array}{ccc}
\cos \theta & 0 & -\sin \theta \\
\sin \phi \sin \theta & \cos \phi & \sin \phi \cos \theta \\
\cos \phi \sin \theta & -\sin \phi & \cos \phi \cos \theta
\end{array}\right]\left[\begin{array}{l}
a_{x} \\
a_{y} \\
a_{z}
\end{array}\right]_{(\psi)}
$$

Upon inspection of Equation (6.11), the first equation of the above set, is only a function of one of the Euler angles, pitch $(\theta)$. An algebraic solution is given as:

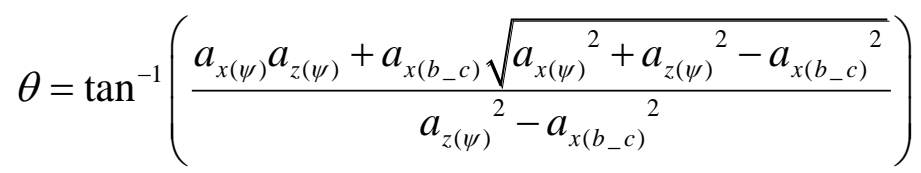


By again rotating the intermediate acceleration vector, $a_{(\psi)}$, with the pitch estimate obtained from Equation (6.12), a second intermediate acceleration vector, denoted with subscript $(\psi, \theta)$, leaves the roll angle as the only unknown variable,

$$
\left[\begin{array}{l}
a_{x} \\
a_{y} \\
a_{z}
\end{array}\right]_{\left(b_{-} c\right)}=\left[\begin{array}{ccc}
1 & 0 & 0 \\
0 & \cos \phi & \sin \phi \\
0 & -\sin \phi & \cos \phi
\end{array}\right]\left[\begin{array}{l}
a_{x} \\
a_{y} \\
a_{z}
\end{array}\right]_{(\psi, \theta)}
$$

, which can be algebraically solved using:

$$
\phi=\tan ^{-1}\left(\frac{-a_{y(\psi, \theta)} a_{z(\psi, \theta)}-a_{y\left(b_{-} c\right)} \sqrt{\left.a_{y(\psi, \theta)}{ }^{2}+a_{z(\psi, \theta)}{ }^{2}-a_{y\left(b_{-} c\right)}\right)^{2}}}{a_{z(\psi, \theta)}{ }^{2}-a_{y\left(b_{-} c\right)}{ }^{2}}\right)
$$

the GPS acceleration vector is calculated using a numerical backward-difference derivative of the GPS velocity measurement vector. To reduce the noise associated with the numerical derivative, the pitch and roll estimates obtained with the AVAE formulation are then smoothed with a first order low-pass Butterworth filter.

\subsection{GPS/INS + Magnetometer Sensor Fusion Formulations}

A triad of orthogonal magnetometers mounted in the aircraft body-axis provides measurement of the magnetic field, which is directly comparable to the known magnetic field as reported by the National Oceanic and Atmospheric Administration (NOAA) [88]. However, in order to relate the body-axis magnetic field readings to the WMM values that are in a local 
coordinate system, a DCM that is function of the aircraft attitude is needed to rotate the WMM values into the aircraft body-axis.

$$
\left[\begin{array}{l}
M_{x} \\
M_{y} \\
M_{z}
\end{array}\right]_{B}=\left[\begin{array}{ccc}
\cos \psi \cos \theta & \sin \psi \cos \theta & -\sin \theta \\
-\sin \psi \cos \phi+\cos \psi \sin \theta \sin \phi & \cos \psi \cos \phi+\sin \psi \sin \theta \sin \phi & \cos \theta \sin \phi \\
\sin \psi \sin \phi+\cos \psi \sin \theta \cos \phi & -\cos \psi \sin \phi+\sin \psi \sin \theta \cos \phi & \cos \theta \cos \phi
\end{array}\right]\left[\begin{array}{c}
M_{x} \\
M_{y} \\
M_{z}
\end{array}\right]_{L}
$$

This provides an additional vector to reference in order to improve attitude estimation. It should be noted that the DCM in Equation (6.15), is simply the transpose of the DCM that was used to rotate acceleration from the body-axis to the local navigation frame, Equation (6.5), as expected.

By providing another information source, magnetometers, present the beneficial scenario in that any two of the three, or all three of the information sources (GPS/Accelerometers, Rate Gyroscopes, Magnetometer) may be combined to provide a fused attitude solution. This benefit is exploited in this study to provide multiple sensor fusion attitude estimation as discussed in Chapter 9 of this dissertation, and to develop a fault-tolerant attitude estimation algorithm as discussed in Chapter $\mathbf{1 0}$ of this dissertation. However, due to hard-iron and soft-iron field disturbances [7], the low-cost magnetometer information is hardly useable without calibration. Therefore, magnetometer calibration that uses a GPS/INS attitude solution is discussed in Chapter 7 of this dissertation.

\subsubsection{3-State INS/Magnetometer Attitude Estimation}

Another 3-state, $\mathbf{x}=\left[\begin{array}{lll}\phi & \theta & \psi\end{array}\right]^{T}$, attitude estimation solution is possible that uses only information from the tri-axial rate gyroscopes and magnetometers. In this formulation, the 
attitude states are predicted using the rate gyroscopes, $\mathbf{u}=\left[\begin{array}{lll}p & q & r\end{array}\right]_{B}^{T}$ by integrating them through the relationships of Equations (5.(2-3)), and the magnetic field vector is used in the measurement update procedure to regulate attitude error growth, $\mathbf{z}=\left[\begin{array}{lll}M_{x}^{B} & M_{y}^{B} & M_{z}^{B}\end{array}\right]^{T}$. In this scenario, the DCM that transforms the body-axis magnetic measurements to the local navigation frame, as shown in Equation (6.15) is used as the observation function that is a function of the predicted attitude states, and the local magnetic field values.

\subsubsection{2-State GPS/Magnetometer Attitude Estimation}

It is also possible to estimate the pitch and roll angles, $\mathbf{x}=\left[\begin{array}{ll}\phi & \theta\end{array}\right]_{B}^{T}$, without the use of the lowcost rate gyroscopes. In this scenario, a reasonable prediction is offered by simply using random walk for prediction

$$
\left[\begin{array}{c}
\dot{\phi} \\
\dot{\theta}
\end{array}\right]=\left[\begin{array}{l}
0 \\
0
\end{array}\right]+w_{r w}
$$

where utilizing random walk for prediction is essentially modeling attitude prediction as the most recent 'a posteriori' estimate with an increased level of uncertainty. Next, the pitch and roll estimates are used with an estimate of the aircraft heading angle that is calculated using the instantaneous arctangent of the GPS velocity, just as in the AVAE formulation presented above.

$$
\psi=\tan ^{-1}\left(\frac{V_{y}}{V_{x}}\right)_{L}
$$


in order to form the DCM that transforms the body-axis measured magnetic vector, $\mathbf{z}=\left[\begin{array}{lll}M_{x}^{B} & M_{y}^{B} & M_{z}^{B}\end{array}\right]^{T}$, to the local navigation coordinate system in order to be directly comparable with the WMM magnetic field values.

\subsubsection{3-State GPS/Accelerometer/Magnetometer Attitude Estimation}

An additional attitude estimation formulation can be considered that references both the Earth's gravity and magnetic field vectors, without using the rate gyroscopes, by using the random walk prediction is again used;

$$
\left[\begin{array}{c}
\dot{\phi} \\
\dot{\theta} \\
\dot{\psi}
\end{array}\right]=\left[\begin{array}{l}
0 \\
0 \\
0
\end{array}\right]+w_{r w}
$$

However, instead of using only GPS to provide an estimate of heading, as in the previous formulation, the observation function is composed of both the DCM that transforms from the body-axis to the local navigation frame, Equation (6.5), for the accelerometers, and its transpose, Equation (6.15), for the magnetometers.

\subsubsection{3-State GPS/INS/Magnetometer Attitude Estimation}

In order to take advantage of all three multiple sources of information, another 3-state: $\mathbf{x}=\left[\begin{array}{lll}\phi & \theta & \psi\end{array}\right]^{T}$, formulation that uses the rate gyroscopes for prediction, and references both the Earth's gravity vector and the Earth's magnetic field vector to regulate error growth is also considered. Where both the GPS velocity measurements and tri-axis accelerometers are used to 
reference the gravity vector, and the magnetometers are used to reference the magnetic field vector. Where, the input vector to the prediction is the rate gyroscope measurements: $\mathbf{u}=\left[\begin{array}{ccc}p & q & r\end{array}\right]_{B}^{T}$, which are integrated over time using Equations (6.2-4), and the observation functions consist of Equation (6.5) and Equation (6.7) with a measurement vector consisting of acceleration calculated by differentiating GPS velocity measurements and the WMM magnetic field values: $\mathbf{z}=\left[\begin{array}{llllll}a_{x}^{G P S} & a_{y}^{G P S} & a_{z}^{G P S} & M_{x}^{B} & M_{y}^{B} & M_{z}^{B}\end{array}\right]^{T}$.

\subsection{Sensor Fusion Formulation Noise Assumptions}

To ensure that performance comparisons were consistent for EKF and UKF, the same assumptions were used to assign process-noise covariance, $Q$, and measurement-noise covariance, $R$, matrices. In general, several different Kalman filter tuning procedures may be adopted, including on-line strategies $[89 ; 48 ; 90 ; 27 ; 37]$. Maybeck, in his classic textbook refers to filter tuning as a process that can be iterative in nature to obtain best performance, often relies on physical insight as to what the noise terms are representing, and in practice "manual optimization" if often used in practice [37]. One approach that is becoming more common within for GPS/INS sensor fusion is to obtain a complete stochastic model of the inertial sensors based on interpreting an Allan deviation curve $[91 ; 4 ; 92 ; 93]$. However, for this study, the most important criterion for filter tuning was to ensure that the procedure was consistent between EKF and UKF, so that the performance comparisons were fair. Therefore, a simple tuning procedure for modeling uncertainty was used that is based on sensor-level measured covariance values.

At high-level, two general approaches were considered for including uncertainty models in the nonlinear Kalman filters: additive noise, and non-additive noise. In Chapter 5, the distinction between assuming additive noise and non-additive noise was made to the extent two 
different versions of both of nonlinear Kalman filtering approaches were presented and discussed. This purpose of focusing on this distinction was due to the fact that in this study both noise assumptions are considered for each of the sensor fusion formulations. Additive process noise lumps uncertainty at the state-level, while non-additive noise provide the freedom for the assumed noise to be used in any manner desired within the nonlinear state-transition and observation functions. The specifics of the approach used in this study for assigning the $Q$ and $R$ matrices both when considering additive noise and non-additive noise assumption are described in this section.

Whenever the sensor fusion formulations are assumed to have additive process-noise, the process-noise covariance matrix associated with the prediction, $Q$, was populated with the variances of each respective IMU channel output, while the plane was static on the runway. As an example, the process-noise matrix of the 9-state sensor fusion formulation is shown in Equation (6.19).

$$
Q=\left(T s^{2}\right) \operatorname{diag}\left(0,0,0, \sigma^{2}\left(a_{x}\right), \sigma^{2}\left(a_{y}\right), \sigma^{2}\left(a_{z}\right), \sigma^{2}(p), \sigma^{2}(q), \sigma^{2}(r)\right)
$$

where the discrete sampling time was included to account for the uncertainty after time integration. As an example consider the roll state estimate. That is, the variance of the roll-rate sensor output, $p$, in units of $(\mathrm{deg} / \mathrm{s})^{2}$ is assumed to project an uncertainty on the roll state estimate in $(\mathrm{deg})^{2}$, therefore the discrete sampling time should be included to represent time-integration. When assuming additive noise, the uncertainty is approximated on the state.

When considering non-additive noise, the tuning approach was again related to the sensor-level variances, similar to the additive noise assumption; however, these variances were used to model uncertainty on the sensor inputs that were used in the nonlinear state-transition 
and observation functions. For example, again when considering the example of the 9-state GPS/INS formulation, the noise was modeled as shown in Equation (6.20)

$$
\mathbf{x}_{k \mid k-1}=f\left(x_{k \mid k-1}, \mathbf{u}_{k}+w_{k}\right)
$$

where $w_{k}$ is comprised of the standard deviations of the IMU sensor outputs when static for a period of time.

$$
w_{k}=w_{0}=\left[\begin{array}{llllll}
\sigma\left(a_{x}\right) & \sigma\left(a_{y}\right) & \sigma\left(a_{z}\right) & \sigma(p) & \sigma(q) & \sigma(r)
\end{array}\right]^{T}
$$

In the case of the UKF, the system was augmented with the process-noise and was directly considered on the IMU input vector. In the case of the EKF, an additional Jacobian was calculated about the inputs, and was used to propagate the process-noise portion of the predicted error-covariance. In Equation (6.21) $w_{k}=w_{0}$, implies that in the formulations considered in this study, the uncertainty of the sensors is not assumed to be time-varying. The non-additive noise approach implicitly considers the discrete sampling time.

A similar approach was used to assign the additive measurement-noise covariance matrix, $R$. These values were approximated by taking the variance of the GPS measurements while the SUAV was at rest on the runway. As an example, of the $R$ matrix for the 9-state formulation is shown in Equation (6.22).

$$
R=\operatorname{diag}\left(\sigma^{2}(x), \sigma^{2}(y), \sigma^{2}(z), \sigma^{2}\left(V_{x}\right), \sigma^{2}\left(V_{y}\right), \sigma^{2}\left(V_{z}\right)\right)
$$

For the 9-state formulation, only additive measurement was considered. This is because the external measurement is a direct measurement of a subset of the observation function (i.e. position and velocity in the local navigation frame). However, this is not the case for the 3 -state GPS/INS sensor formulation. In the 3-state formulation, the innovation residual is the difference between acceleration in the local navigation frame as measured by GPS and as predicted given 
the predicted attitude states and acceleration information. Therefore, when additive noise is assumed in the 3-state formulation, the uncertainty attributed to both the accelerometers and the GPS velocity measurements are lumped into a single 3x3 error covariance matrix $R$.

$$
R_{3 \times 3}=R_{G P S_{V x, y, z}}+R_{a c c e l}
$$

On the contrary, when considering the more general case of non-additive noise, the uncertainty of the two sensors is decoupled. That is, the measurement-noise is assumed to be

$$
\begin{aligned}
& v_{k}=v_{0}=\left[\begin{array}{llllll}
\sigma\left(a_{x}\right) & \sigma\left(a_{y}\right) & \sigma\left(a_{z}\right) & \sigma\left(V_{x}\right) & \sigma\left(V_{y}\right) & \sigma\left(V_{z}\right)
\end{array}\right]^{T} \\
& R=E\left[v_{k} v_{k}^{T}\right] \\
& R=\left[\begin{array}{cc}
R_{\text {accel }} & 0 \\
0 & R_{G P S_{V x, y, z}}
\end{array}\right]
\end{aligned}
$$

where for the UKF each of the of the terms of $v_{\mathrm{k}}$ in the augmented state vector are used to include uncertainty at the sensor level, and for the EKF an additional Jacobian about this assumption is used to propagate the measurement-error covariance matrix when calculating the Kalman gain.

In general, as discussed in Chapter $\mathbf{5}$ process-noise and measurement-noise do not only represent physical sensor noises, but also uncertainty in the state-transition and observation models $[37 ; 24]$. For the purposes of this study, the state-transition and observation functions are kinematic relationships, which are theoretically correct [24]. Therefore, there is little uncertainty in the model, and the simple approach to filter tuning that is related to sensor-level noise is justifiable. For the non-additive case, the introduced uncertainty in the filter comes from sensors, and is modeled as such. 


\section{Chapter 7 : Sensor Fusion with Sensor Calibration}

\subsection{State and Parameter Estimation}

For state-estimation when employing a nonlinear estimator as discussed in Chapter 5, system parameters are assumed to be known, however there are many cases where parameters are not known. Adaptive filtering techniques consider estimating unknown parameters and/or stochastic noise assumptions on-line, in addition to the original states [25;23].

State and parameter estimation approaches that are based on KF fall into two broad categories, namely dual estimation and joint estimation [81]. The dual estimation approach considers using a filter for state estimation $\mathrm{KF}$ in parallel with a $\mathrm{KF}$ that considers parameter estimation, and the joint estimation approach considers augmenting the state vector with the set of parameters that are to be estimated, and considering them in a single estimation filter. As Stengel notes in his text, successful parameter estimation depends on "the number of uncertain parameters, the magnitude of uncertainty, the functional dependence of outputs on the uncertain parameters, and the knowledge of the system inputs" [25].

For joint estimation with the augmented system, $\mathbf{x}^{a}=\left[\begin{array}{ll}\mathbf{x}(t) & \mathbf{p}(t)\end{array}\right]$, the normal predictorcorrector framework is followed, it possible to include additional dynamic equations to predict the parameters. In this approach the filter attempts to minimize the error around the entire augmented state vector. For the dual estimation approach, the state and parameters are communicated between the two parallel filters, however the covariance between states and parameters are assumed to be statistically uncorrelated [32]. That is, in the joint estimation approach, the error covariance matrix is written as shown in Equation (7.1) 


$$
P=\left[\begin{array}{cc}
P_{\mathbf{x}} & P_{\mathbf{p}, \mathbf{x}} \\
P_{\mathbf{x}, \mathbf{p}} & P_{\mathbf{p}}
\end{array}\right]
$$

where, $\mathbf{x}$, refers to the original state vector, and ,p, refers to the additional parameters. In the dual estimation approach, the off-diagonal terms of Equation (7.1) are assumed to be zero [32].

In this study, both dual estimation and joint estimation approaches were considered for the purpose of tracking biases IMU on-line; however, it was determined that assuming that the cross-terms (i.e. off-diagonal) of error-covariance matrix between the time-varying biases and original states were zero, as discussed In Equation (7.1), was an invalid assumption for this problem. Specifically, these cross-covariance terms were found to have a significant magnitude in comparison to the diagonal terms of the error-covariance matrix, and to therefore significantly contribute to calculation of the Kalman gain. The results of this analysis are included in Appendix B of this dissertation. Therefore, in this study, when considering additional on-line sensor bias estimation, the state vector of the sensor fusion formulation was augmented to include the additional IMU bias states, and the joint estimation filter approach was used.

\subsection{Random Walk Assumption for On-Line IMU Calibration}

In this study, a simple generic error-model for the sensor bias was considered,

$$
b_{\text {total }}=b_{D C}+b(t)
$$

,where $b_{D C}$ is the static sensor bias that is easy to determine by monitoring the sensor outputs while the sensor is static. For each of the four IMU's that were used in the flight data library, a single set of static biases were approximated. Specifically, these values were determine by using 
the average of the sensor outputs of each IMU during a period that the SUAV was static on the runway within the data set of the first chronological flight for each IMU. Therefore only was set of static biases were used for each IMU, and they were not changed from flight-to-flight.

The GPS/INS and GPS/INS/Magnetometer formulations discussed in Chapter 5 only considered the $b_{D C}$ to handle IMU sensor biases and magnetometer biases, using the values that were determined off-line. However, in this study, an approach that also attempts to use sensor fusion to account for the time-varying bias, $b(t)$, on-line is considered. In order to do this, the random walk dynamic model was used. The random walk assumption is one that several others have adopted for handling time-varying biases of low-cost MEMS IMUs [32; 51]

$$
\dot{b}(t)=0+\mathbf{w}_{b}
$$

where the bias states were perturbed by small magnitude white noises. The actual variance of the time-varying portion of the sensor bias is rarely reported by low-cost IMU manufacturers [40], and in this study, the magnitude of these white noise values were assigned to be the same for all four IMUs, and 'tuned' for estimation performance. Simon [23], refers to this noise as "artificial noise" that allows the nonlinear KF to track the unknown parameter when minimizing its cost function. It should be noted that for INS applications, this noise has a physical meaning and in fact comprehensive stochastic modeling of the inertial sensor errors is possible $[4 ; 94 ; 92$; 93], but this is outside the scope of this study. 


\subsection{Sensor Fusion with On-Line Calibration}

\subsubsection{9-State Position, Velocity and Attitude + 6- IMU Bias Estimation}

A 9-state + 6- IMU bias GPS/INS sensor fusion formulation is included by augmenting the 9state solution to further allow the nonlinear estimator to account for time-varying biases on the IMU accelerometer and rate gyros. Therefore, the augmented state vector includes the original PVA states and six sensor biases

$$
\mathbf{x}=\left[\begin{array}{lllllllllllllll}
x & y & z & V_{x} & V_{y} & V_{z} & \phi & \theta & \psi & b_{a_{x}} & b_{a_{y}} & b_{a_{z}} & b_{p} & b_{q} & b_{r}
\end{array}\right]^{T} \text {. For this GPS/INS }
$$

formulation the prediction procedure is the same to account for the first nine state update relationships as the 9-state formulation, however the bias states are used to correct the raw IMU measurements, that is:

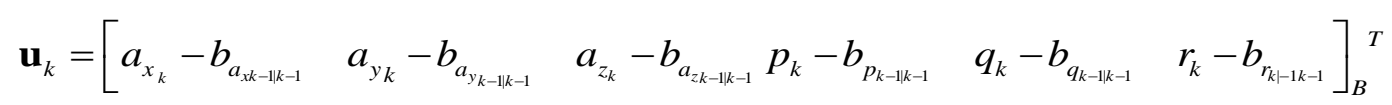

For the remaining six bias states, the model presented in Equation 6.3 is used to perturb the bias state. As the 3 and 9-state formulations, the required Jacobian matrices for a 9-state +6-IMU bias EKF are included in Appendix A.

\subsubsection{3-State Attitude + 6 IMU Bias Estimation}

Just as the 9-state formulation was augmented, it is also possible to augment the 3-state attitude estimation formulation to account for time-varying IMU sensor biases. In this case the augmented state and vector is defined as $\mathbf{x}=\left[\begin{array}{lllllllll}\phi & \theta & \psi & b_{a_{x}} & b_{a_{y}} & b_{a_{z}} & b_{p} & b_{q} & b_{r}\end{array}\right]^{T}$, where the 
input to the state-transition function is $\mathbf{u}_{k}=\left[\begin{array}{lll}p_{k}-b_{p k-1 \mid k-1} & q_{k}-b_{q_{k-1 k-1}} & r_{k}-b_{r k-1 \mid k-1}\end{array}\right]_{B}^{T}$ and the input to the nonlinear observation function is $\mathbf{c}_{k}=\left[\begin{array}{llll}a_{x_{k}}-b_{a_{k-k-k-1}} & a_{y_{k}}-b_{a_{y k-1 \mid k-1}} & a_{z_{k}}-b_{a_{z k-1 \| k-1}}\end{array}\right]_{B}^{T}$.

\subsubsection{3-State GPS/INS/Mag. Attitude + 6 IMU Bias + 3 Mag. Bias}

In order to take advantage of all of the information, and also allow the estimation filter to track biases on-line, another formulation was formulated to estimate 3 states and 9 time-varying parameters. The nine parameters consist of the six IMU biases (i.e. 3 accelerometers, 3 rate gyroscopes) and 3 residual magnetometer biases. Therefore the augmented state vector of this formulation is $\quad \mathbf{x}=\left[\begin{array}{llllllllllll}\phi & \theta & \psi & b_{a_{x}} & b_{a_{y}} & b_{a_{z}} & b_{p} & b_{q} & b_{r} & b_{M x} & b_{M z} & b_{M z}\end{array}\right]^{T}$. The nonlinear prediction function, $f$, and observation function, $h$, for this formulation is the same at the 3 -state GPS/INS/Mag. formulation described in Section 6.3.4, but the rate gyroscopes, accelerometer and magnetometers have the biases applied to them whenever they appear in $\mathbf{u}_{k}, \mathbf{c}_{k}$, and $\mathbf{z}_{k}$ respectively.

\subsection{Magnetometer Calibration with GPS/INS}

Un-calibrated magnetometers provide a distorted measurement of the Earth's magnetic field due to local field disturbances [7; 95]. For example, in the case of a SUAV, ferromagnetic materials in the onboard electronic payload may cause a disturbance of the local magnetic field. Within this study, a magnetometer calibration method is formulated that relies on the solution of the aircraft attitude derived from a GPS/INS sensor fusion algorithm.

To derive a calibration model for the magnetometers, nine parameters are used to define the magnetometers bias, scale factor, and non-orthogonality terms. 


$$
\left[\begin{array}{c}
\hat{M}_{x}^{B} \\
\hat{M}_{y}^{B} \\
\hat{M}_{z}^{B}
\end{array}\right]=R_{M}\left(R_{M \phi}, R_{M \theta}, R_{M \psi}\right) \cdot\left[\begin{array}{ccc}
S_{M x} & 0 & 0 \\
0 & S_{M y} & 0 \\
0 & 0 & S_{M z}
\end{array}\right] \cdot\left[\begin{array}{c}
M_{x}^{B} \\
M_{y}^{B} \\
M_{z}^{B}
\end{array}\right]-\left[\begin{array}{c}
b_{M x} \\
b_{M y} \\
b_{M z}
\end{array}\right]
$$

This magnetometer error model [7; 95]accounts for the combined effects of the soft-iron /hardiron effects as well as the sensors own bias, scale and, and non-orthogonality. In Equation (7.5) $R_{M}$ is a DCM of three rotation angles $R_{M \phi}, R_{M \theta}, R_{M \psi}$ and is defined as:

$$
R_{M}=\left[\begin{array}{ccc}
\mathrm{c}\left(R_{M \psi}\right) \mathrm{c}\left(R_{M \theta}\right) & -\mathrm{s}\left(R_{M \psi}\right) \mathrm{c}\left(R_{M \phi}\right)+\mathrm{c}\left(R_{M \psi}\right) \mathrm{s}\left(R_{M \phi}\right) & \mathrm{s}\left(R_{M \psi}\right) \mathrm{s}\left(R_{M \phi}\right)+\mathrm{c}\left(R_{M \psi}\right) \mathrm{s}\left(R_{M \theta}\right) \mathrm{c}\left(R_{M \phi}\right) \\
\mathrm{s}\left(R_{M \psi}\right) \mathrm{c}\left(R_{M \theta}\right) & \cos \left(R_{M \psi}\right) \cos \left(R_{M \phi}\right)+\mathrm{s}\left(R_{M \psi}\right) \mathrm{s}\left(R_{M \theta}\right) \mathrm{s}\left(R_{M \phi}\right) & -\mathrm{c}\left(R_{M \psi}\right) \mathrm{s}\left(R_{M \phi}\right)+\mathrm{s}\left(R_{M \psi}\right) \mathrm{s}\left(R_{M \theta}\right) \mathrm{c}\left(R_{M \phi}\right) \\
-\mathrm{s}\left(R_{M \theta}\right) & \mathrm{c}\left(R_{M \theta}\right) \mathrm{s}\left(R_{M \phi}\right) & \mathrm{c}\left(R_{M \theta}\right) \mathrm{c}\left(R_{M \phi}\right)
\end{array}\right]_{7.6}
$$

where $\mathrm{c}(-)$ represents cosine and $\mathrm{s}(-)$ represents sine.

Therefore, the set of nine parameters $\Theta_{M}=\left[\begin{array}{lllllllll}R_{M \phi} & R_{M \theta} & R_{M \psi} & S_{M x} & S_{M y} & S_{M z} & b_{M x} & b_{M y} & b_{M z}\end{array}\right]^{T}$ must optimized such that an objective function is minimized between the Earth's magnetic-field values projected into the aircraft body-axis, and the magnetometer measurements obtained in the aircraft body-axis.

$$
J_{M}=\sum\left[\left(M_{x}^{B}-M e_{x}^{B}\right)^{2}+\left(M_{y}^{B}-M e_{y}^{B}\right)^{2}+\left(M_{y}^{B}-M e_{y}^{B}\right)^{2}\right]
$$

In Equation (7.7), in order to project the WMM magnetic field values into the body axis, $M e^{B}$, the solution of the aircraft Euler angles over and entire flight provided by a GPS/INS sensor fusion algorithm were used. Specifically, given the aircraft Euler angles, from GPS/INS the 
relationship of Equation (6.15) was used. To perform this parameter optimization, Matlab's ${ }^{\circledR}$ unconstrained nonlinear optimization was function was used. 


\section{Chapter 8 : GPS/INS Sensor Fusion Performance Comparison}

Some of the results described in this chapter of the dissertation are summarized and presented in a journal article submission [87], and were built upon conference proceedings articles $[89 ; 96]$.

\subsection{Un-aided Low-Cost Navigation}

In order to provide a background for the problem addressed in this study, first some examples of navigation results obtained by only relying on the low-cost inertial sensors are shown. That is, attitude estimates are shown that were obtained by directly integrated MEMS rate gyroscopes, and position and velocity estimates that were obtained by directly integrating MEMS accelerometers and transforming their rotations with the attitude provided by the lowcost rate gyros. To demonstrate these errors, the attitude estimates are compared to the highquality mechanical gyroscope measurements (Goodrich® VG34).

In all of the formulations considered in the chapter the IMU data was processed at $100 \mathrm{~Hz}$, and the GPS information was processed at $20 \mathrm{~Hz}$. This resulted in a 5-to-1 prediction to measurement-update ratio with respect to the nonlinear Kalman Filters.

\subsubsection{Low-Cost Inertial Navigation}

Figures 8.1 and 8.2 demonstrate the large attitude errors present when relying on un-aided low-cost MEMS IMU based attitude estimates. 


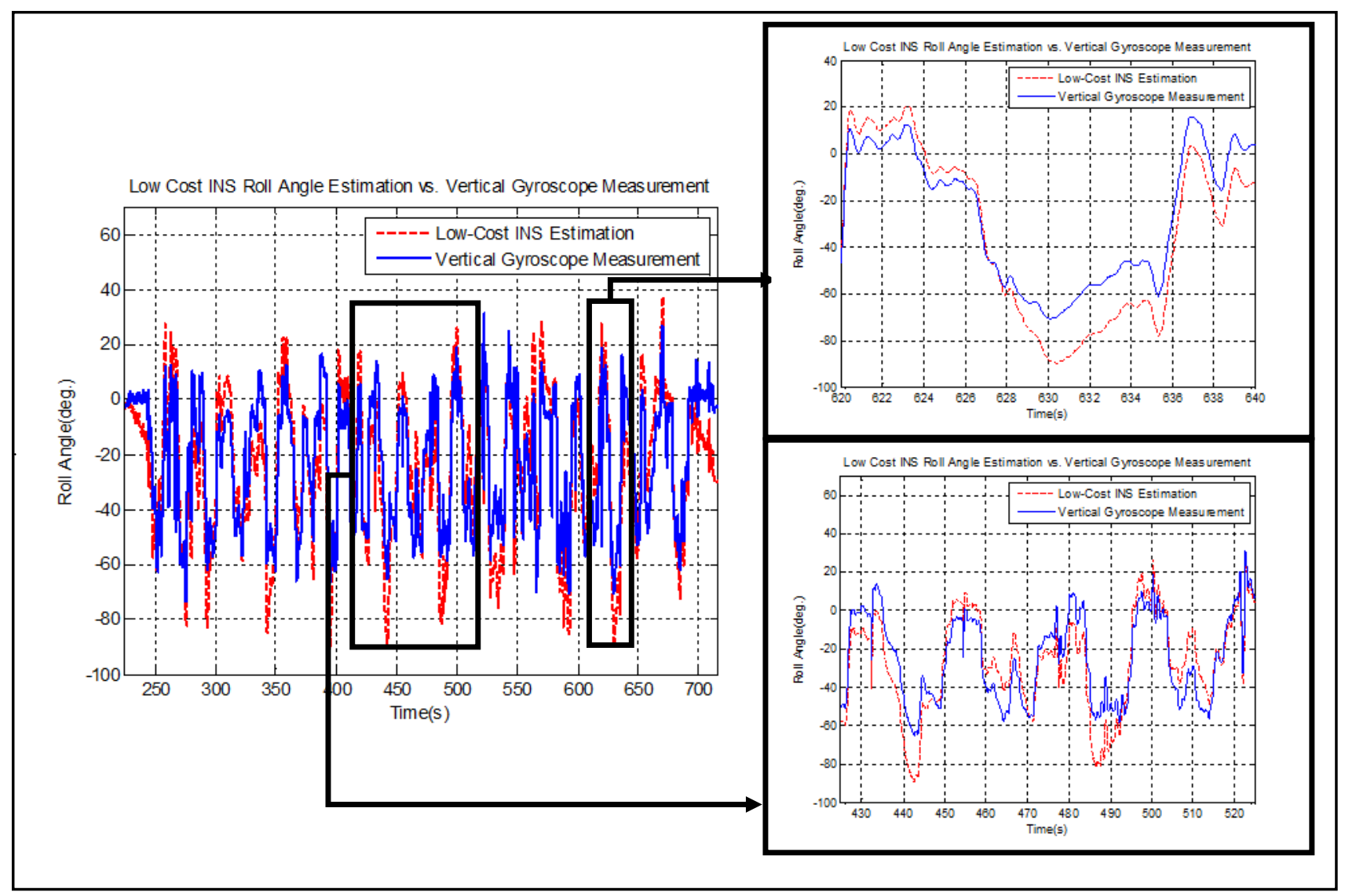

Figure 8.1: Low-Cost IMU Roll Estimation vs. Vertical Gyro Roll Measurement

As shown in Figure 8.1 the MEMS IMU based attitude estimates follow the general of the vertical gyroscope. It is important to note that during this analysis, the IMU's static sensor biases were removed using a simple average of the sensor outputs while the plane was level and at rest on the runway. If the significant static sensor biases were not removed, the attitude estimation error of the low-cost would be even worse due to time integration. Figure 8.2 shows a similar trend with respect to the low-cost IMU pitch estimates. 


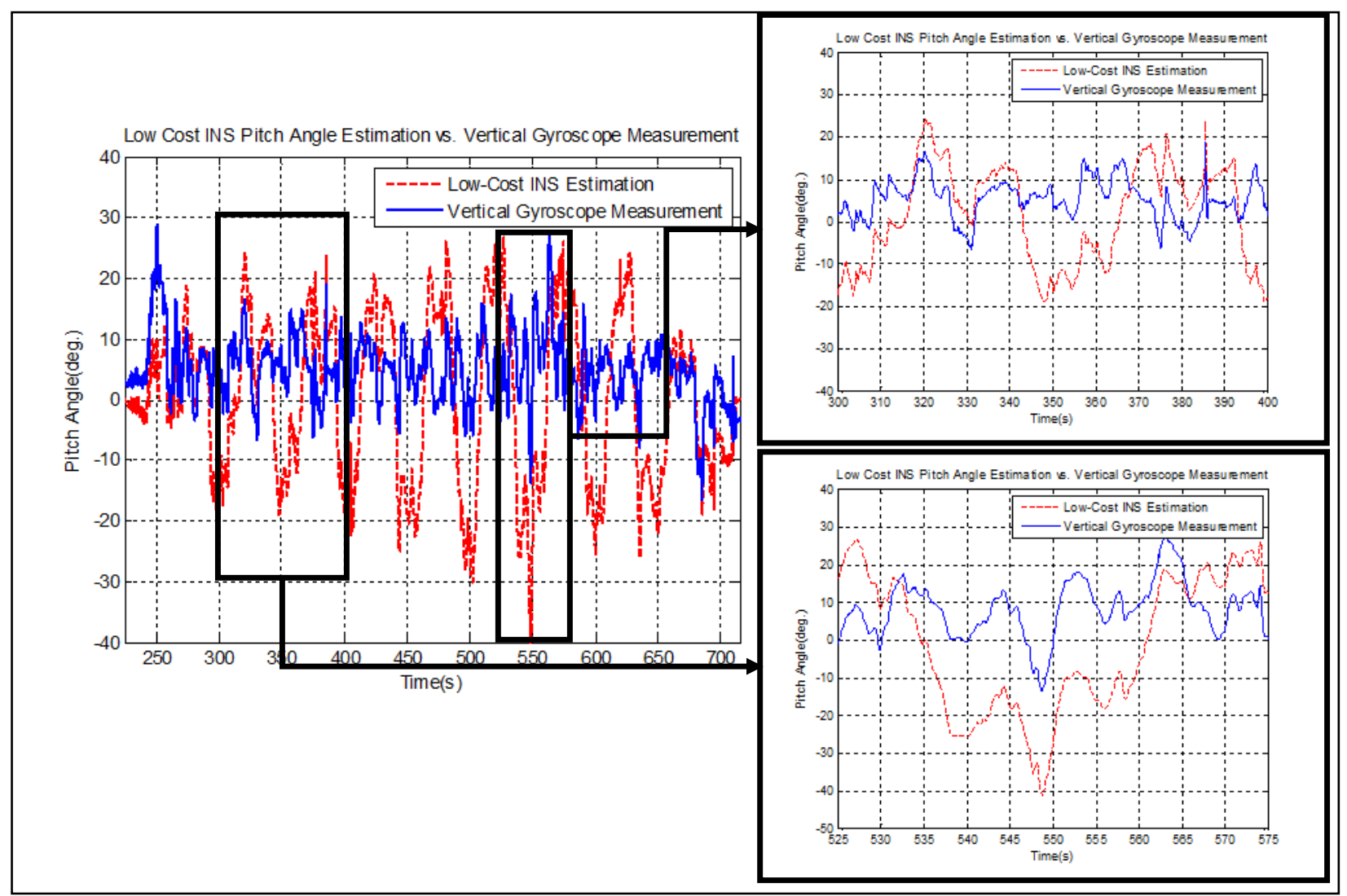

Figure 8.2: Low-Cost IMU Pitch Estimation vs. Vertical Gyro Pitch Measurement

Because the signal to noise ratio of the roll signal is lower than that of the pitch signal, the roll channel appears less noisy, however, upon looking at the zoomed-view on the right of plots in Figures 8-1 and 8-2, it is apparent that the magnitude of the estimation error of the two attitude estimates are similar. The attitude errors present in Figures 8.1 and 8.2 also contribute to the position and velocity drift of the low-cost MEMS navigation solution. In addition, the sensor biases present on the accelerometer outputs causes quick position and velocity drift, as indicated in Figures 8.3 and 8.4 . 

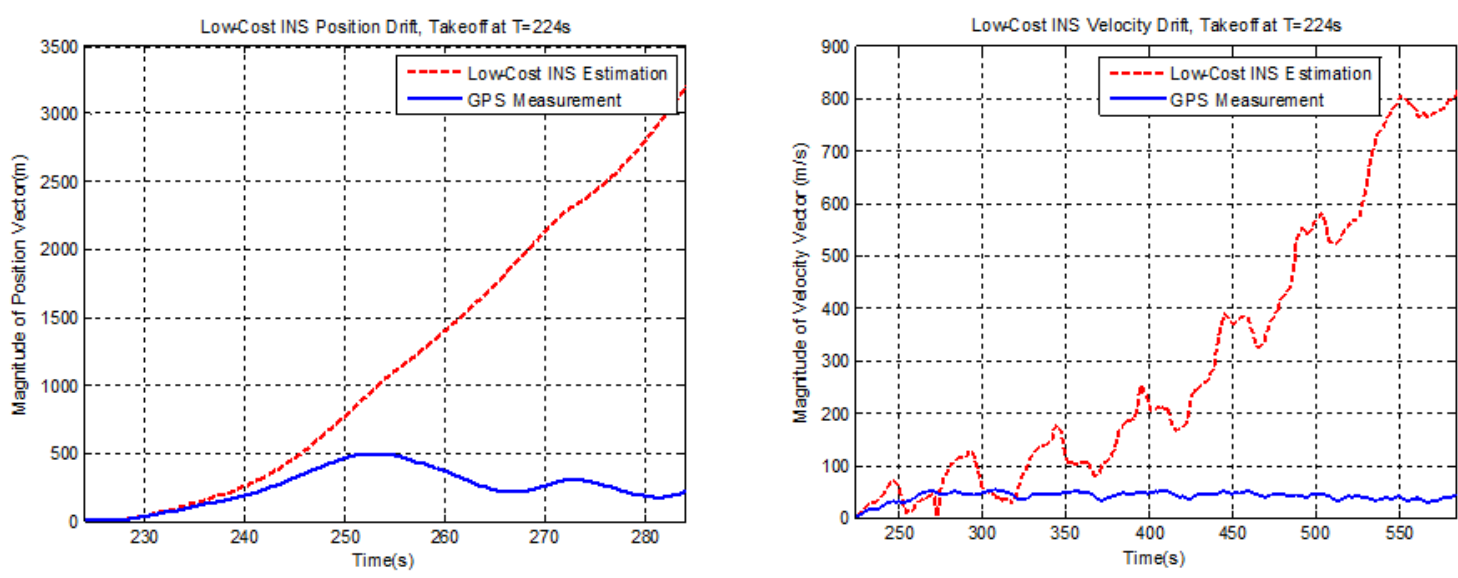

Figure 8.3: Position drift I and velocity drift (L) of Low-Cost INS compared to GPS.

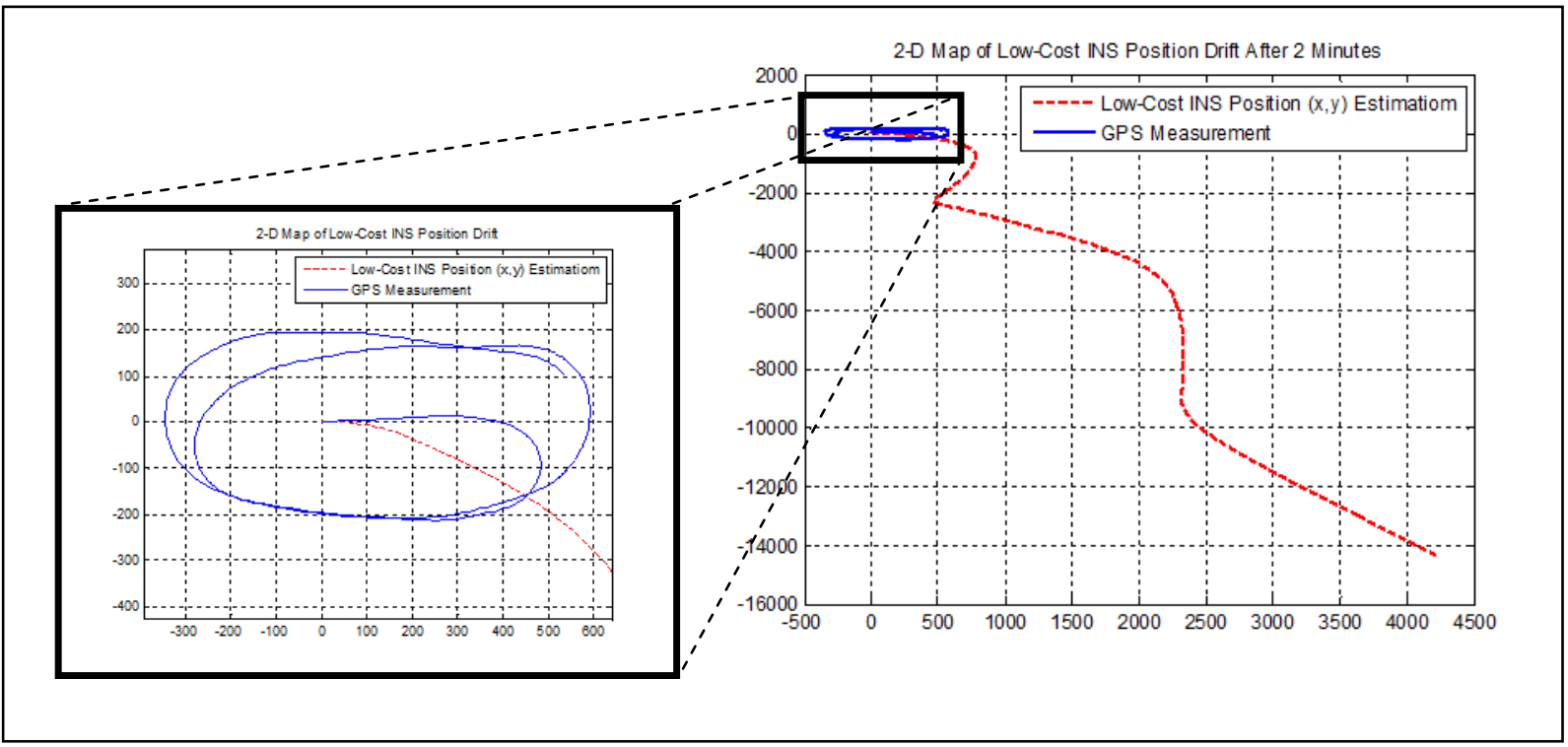

Figure 8.4: A 2-D (x,y) map of low-cost INS position compared to GPS track.

Figures 8.3 and 8.4 illustrate the fact that stand-alone low-cost IMU based position and velocity solutions are hardly useable. Figure 8.3 shows an overall position drift of around 3000 meters one minute after take-off, and an estimated velocity magnitude that is around 16 the actual 
SUAV velocity. Figure 8.4 is a different representation of position drift, in which the flight is shown from take-off until a few laps of loiter, and the estimated position drifts just after take-off.

\subsubsection{GPS Only Navigation}

While providing a statistically unbiased measurement that does not grow with time, relying on a GPS receiver alone also presents some problems. First, a single GPS antenna receiver cannot be used to determine the aircraft attitude, and accurate knowledge of aircraft attitude is critical in flight control applications. Additionally, GPS position and velocity measurements are often corrupted with high frequency noise due to antenna shielding. Figure 8.5, provides an example of a spike present in the GPS measurement.

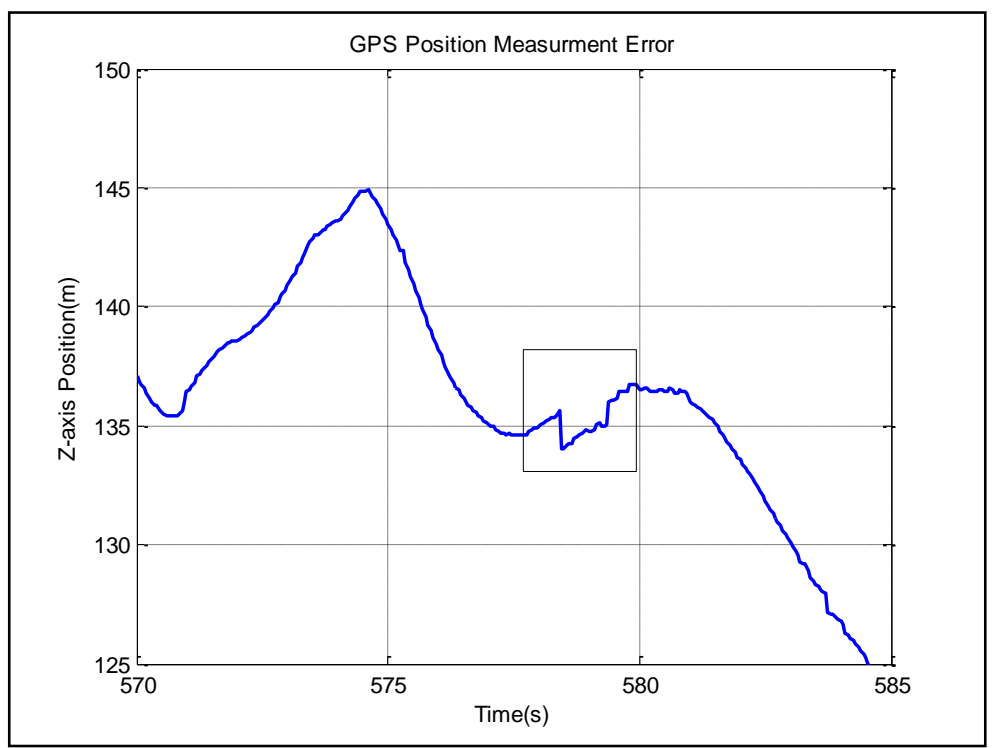

Figure 8.5: An example of poor GPS position measurement.

As apparent in Figure 8-5, the GPS Z-axis measurement has a significant jump on the order of 1 meter over a single measurement time-step $(20 \mathrm{~Hz})$. While the focus of this dissertation is attitude estimation, largely due to the availability of independent attitude truth data, qualitative assessments of the sensor fusion estimations ability to smooth GPS measurement error are shown. 


\subsection{GPS/INS Sensor Fusion Comparison Study Flight Data Library}

For this GPS/INS comparison study, 23 sets of YF-22 flight data were used. In particular:

- 8 Flights on the Green YF-22

- 5 Flights on the Red YF-22

- 8 Flights on the Blue YF-22

- 2 Flights on the Blue YF-22* (with Gen-V Avionics System)

Within these flights, a total of four different electronic instrumentation payloads were used. Specifically, three different FFASs were used on each of the three YF-22s (Blue, Red, Green), and the new Gen-V avionics system was retro-fit on the Blue YF-22 research UAV (indicated as Blue YF-22*). A distribution of selected flight data with respect to flight speed and GPS attitude is shown in Figure 8.6.

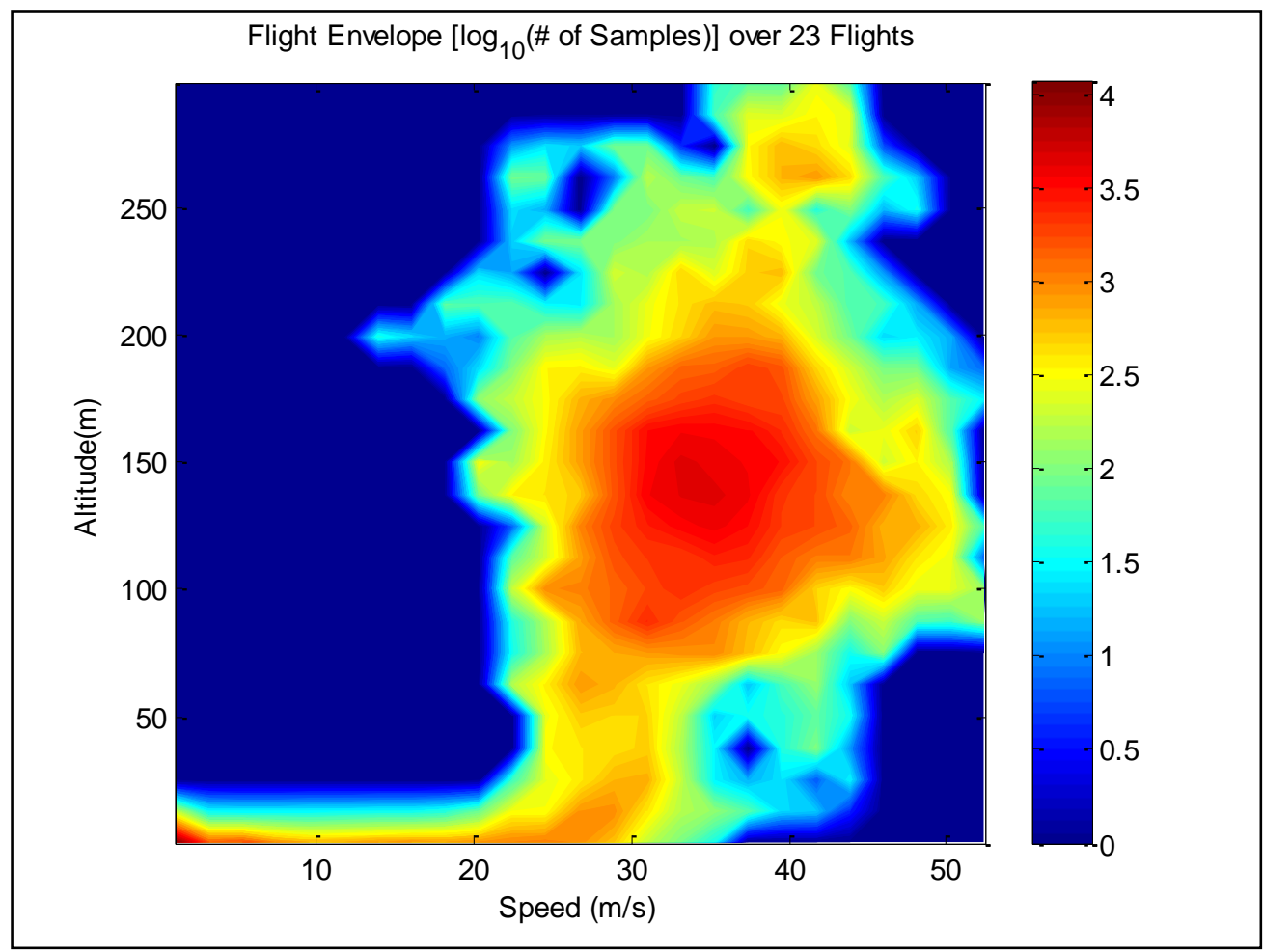

Figure 8.6 Flight data envelope with respect to speed and altitude. 
Over the 23 flights, the average GPS speed was $71.2 \mathrm{mph}$, and the average attitude was around 150 meters. Furthermore, the outdoor temperature during the various 23 flights varied from a minimum of $38^{\circ} \mathrm{F}$ to a maximum of $80^{\circ} \mathrm{F}$, with a mean temperature of $62.9^{\circ} \mathrm{F}$ and a standard deviation of $14.1^{\circ} \mathrm{F}$. The contour in Figure 8.7 shows the distribution of all data samples with respect to pitch and roll angles, indicating dynamic flight conditions and a large flight envelope covered over the 23 flights.

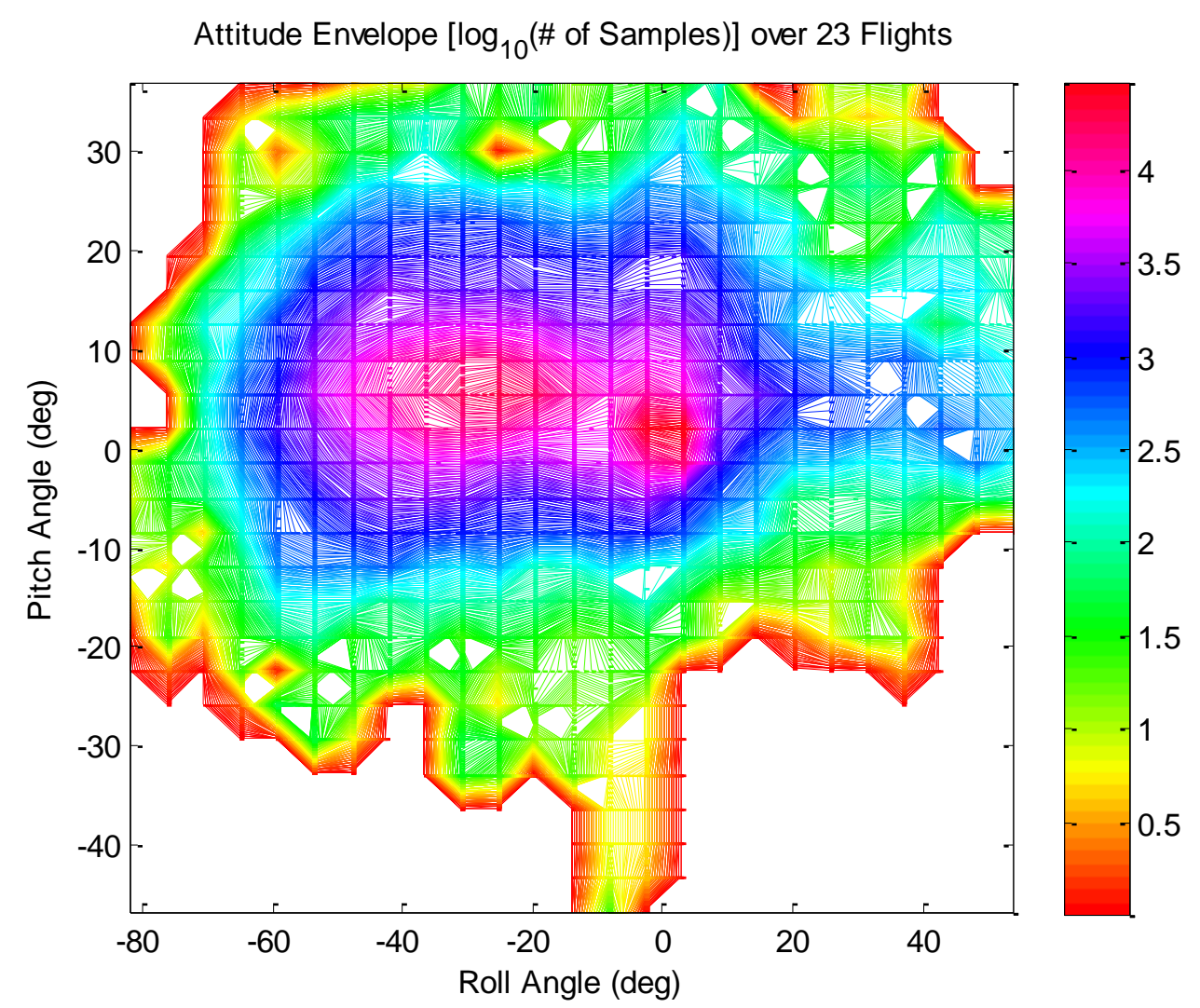

Figure 8.7: Distribution of aircraft attitude samples over 23 flights [87].

Figure 8.7 shows a dense amount of data clustered around approximately negative $30^{\circ}$ roll angle, which is attributed to the fact the most of the flights are in a loitering patter with left hand turns. 
The average roll angle over the 23 flights was $-19.8^{\circ}$, and the standard deviation was $20.64^{\circ}$. In terms of pitch, the average aircraft pitch angles was $5.39^{\circ}$ and the standard deviation of pitch was $6.78^{\circ}$. The total duration of flight data considered for this attitude estimation comparison is 3 hours 9 minutes and 41 seconds, with average flight duration of 8 minutes and 15 seconds.

\subsection{EKF and UKF Comparison for Loosely Coupled GPS/INS Sensor Fusion}

\subsubsection{Performance Evaluation Metrics}

For this analysis a performance index, $J$, was selected to reflect the composite estimation error in units of degrees. Therefore, this value includes the combination of both the mean absolute error and error standard deviation on both roll and pitch angle axes with respect to the Goodrich® mechanical vertical gyroscope that was used as the independent 'truth' data.

$$
J=w_{\text {mean }}\left(\left|\phi_{\text {est }}-\phi_{\text {truth }}\right|+\left|\theta_{\text {est }}-\theta_{\text {truth }}\right|\right)+w_{\sigma}\left(\sigma\left[\phi_{\text {est }}-\phi_{\text {truth }}\right]+\sigma\left[\theta_{\text {est }}-\theta_{\text {truth }}\right]\right)
$$

In order to maintain units of degrees, the weights in Equation (8.1) were chosen to be $w_{\sigma}=0.3$, $w_{\text {mean }}=0.2$ in order to sum to unity. Choosing $w_{\sigma}=0.3$ places higher emphasis on the standard deviation of the estimation error. In general, a smaller $J$ indicates a better overall estimation performance of a sensor fusion algorithm with respect to the mechanical gyroscope measurements.

Additional values that are reported to evaluate performance are the four terms that are used in the calculation of the performance index, $J$, the standard deviation of the performance index over the 23 flights, and the maximum absolute error on both the roll and pitch channels. 


\subsubsection{Simple Stochastic Sensor Modeling Approach}

To verify the tuning approach used for this study, that were outlined in Chapter $\mathbf{6}$ and Chapter 7 a simple tuning test was performed to evaluate UKF and EKF's sensitivities to these stochastic model parameters. Specifically, a ratio scale factor between the process-noise covariance matrix and measurement-noise covariance matrix , $\gamma$,

$$
Q=Q_{0}, R=\gamma R_{0}
$$

was varied over a large range of values, where a large $\gamma$ represents an increase in the reliance on the filter's prediction step while a small $\gamma$ represents an increase on the reliance of the measurement-update procedure within the Kalman filter. Figure 8.8 shows the estimation performance of the 9-State +6 Bias EKF and UKF as a function of the tuning parameter $\gamma$, averaged over 23 flights.

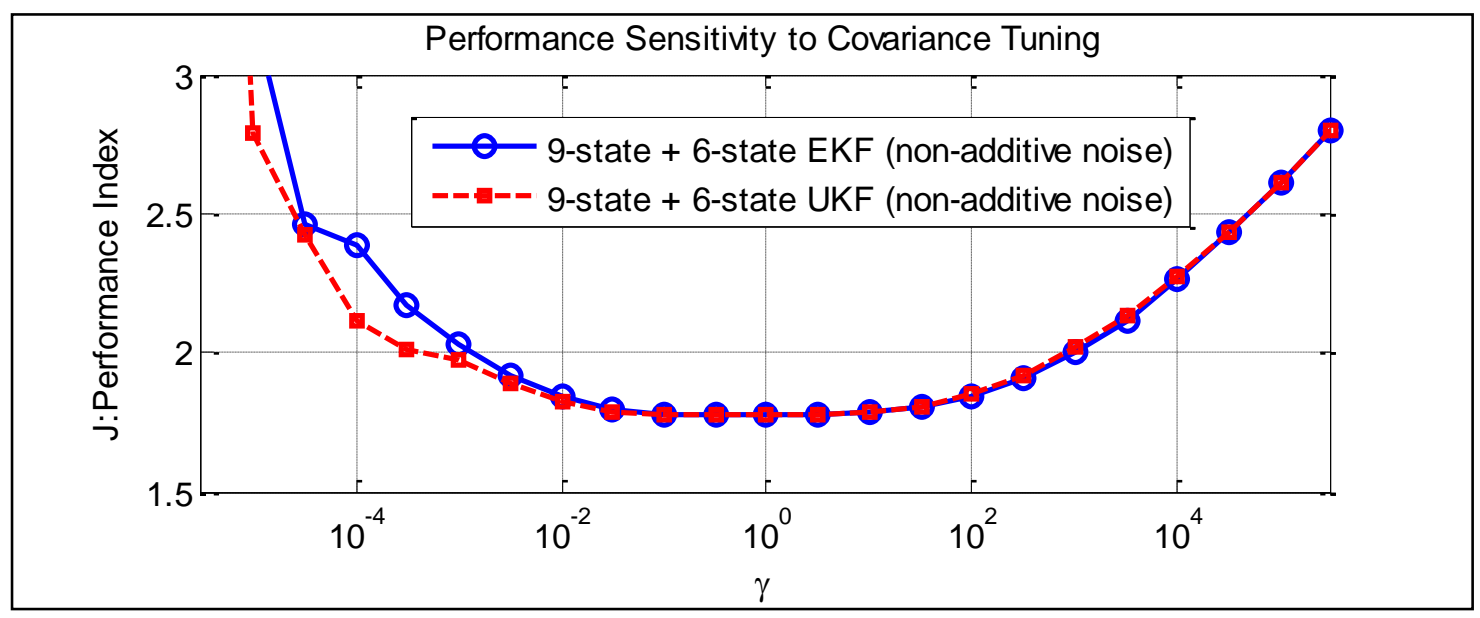

Figure 8.8: Covariance tuning profile of the 9-State+6-Bias EKF and UKF (w/ non-additive noise). 
Figure 8.8 indicates that both EKF and UKF are well tuned. That is, when $\gamma=1$, the performance is close to its overall best performance (minimum $J$ ). In addition, it appears that for this formulation EKF and UKF are similarly sensitive to this covariance tuning parameter. The exception to this is at extremely low $\gamma$ which represents now reliance on external measurements. Additionally, both curves are relatively flat for a large period (e.g. a few orders of magnitude

around zero). This represents that both EKF and UKF provide good performance over a relatively broad range of tuning. For a comprehensive analysis of the EKF and UKF sensitivity to covariance tuning as well as various other design parameters, sampling rate, and initialization error within these specific GPS/INS sensor formulations please refer to Rhudy, Gu, Gross, Gururajan and Napolitano [97].

\subsubsection{Performance Comparison Results}

The discussion of the results of the GPS/INS sensor fusion study starts by considering the average performance over 23 -flights of low-cost INS attitude estimation, as well as the AVAE approach. The AVAE approach is included, although it does not rely on a nonlinear KF, because it poses a cheap solution that significantly reduces estimation error, but it should not be as good in terms of performance or as elegant of a solution as employing the nonlinear KFs. Table 8-1 lists the attitude estimation performance metrics of low-cost INS and AVAE averaged over 23 flights. 
Table 8-1: GPS/INS sensor attitude estimation performance of Low-Cost INS and AVAE

\begin{tabular}{|c|c|c|c|c|c|c|c|c|}
\hline $\begin{array}{c}\text { GPS/INS Sensor } \\
\begin{array}{c}\text { Fusion } \\
\text { Formulation }\end{array}\end{array}$ & $\operatorname{mean}(\mathbf{J})$ & $\boldsymbol{\sigma}(\mathbf{J})$ & $\operatorname{mean}\left(\mid \boldsymbol{\phi}_{\text {err }}\right)$ & $\boldsymbol{\sigma}\left(\boldsymbol{\phi}_{\text {err }}\right)$ & $\max \left(\left|\boldsymbol{\phi}_{\text {err }}\right|\right)$ & $\operatorname{mean}\left(\left|\boldsymbol{\theta}_{\text {err }}\right|\right)$ & $\boldsymbol{\sigma}\left(\boldsymbol{\theta}_{\text {err }}\right)$ & $\max \left(\left|\boldsymbol{\theta}_{\text {err }}\right|\right)$ \\
\hline Low-Cost INS & 12.769 & 11.774 & 11.910 & 14.493 & 41.018 & 11.035 & 12.775 & 29.093 \\
\hline AVAE & 3.698 & 0.304 & 3.200 & 4.680 & 59.830 & 2.765 & 3.669 & 28.686 \\
\hline
\end{tabular}

* All values are in degrees

Table 8-1 reaffirms the preliminary analysis conducted in Section 8.8.1 in that low-cost INS is not viable for attitude estimation. An important indication of this is that the deviation of the estimation performance over the 23 flights, $\sigma(J)$, is nearly as large as the average estimation performance. In addition, the average maximum attitude errors are substantial. However, the AVAE algorithm significantly reduces the error, in terms of average performance index. Additionally, the AVAE performance is quite consistent from flight-to-flight as indicated by $\sigma(J)$. However, the average maximum errors are not reduced with this approach.

Tables 8-2 and 8-3 summarizes the average performance over the 23 flights for each EKF and UKF GPS/INS formulation in terms of average performance index, variation of the performance index and the statistics of the roll and pitch error. Figure, Table 8-2 represents the performance senor fusion algorithms when considering additive process-noise.

Table 8-2: Attitude estimation performance between EKF and UKF assuming additive noise

\begin{tabular}{|c|c|c|c|c|c|c|c|c|}
\hline \multicolumn{7}{|c|}{ Assuming Additive Noise } \\
\hline $\begin{array}{c}\text { GPS/INS Sensor } \\
\text { Fusion Formulation }\end{array}$ & $\operatorname{mean}(\mathbf{J})$ & $\boldsymbol{\sigma}(\mathbf{J})$ & $\operatorname{mean}\left(\mid \boldsymbol{\phi}_{\text {err }}\right)$ & $\boldsymbol{\sigma}\left(\boldsymbol{\phi}_{\text {err }}\right)$ & $\boldsymbol{m a x}\left(\left|\boldsymbol{\phi}_{\text {err }}\right|\right)$ & $\operatorname{mean}\left(\left|\boldsymbol{\theta}_{\text {err }}\right|\right)$ & $\boldsymbol{\sigma}\left(\boldsymbol{\theta}_{\text {err }}\right)$ & $\max \left(\left|\boldsymbol{\theta}_{\text {err }}\right|\right)$ \\
\hline 3-state EKF & 2.065 & 0.224 & 2.244 & 2.200 & 13.216 & 1.869 & 1.940 & 7.780 \\
\hline 3-state UKF & 2.072 & 0.238 & 2.248 & 2.207 & 13.219 & 1.875 & 1.952 & 7.738 \\
\hline 9-state EKF & 2.077 & 0.280 & 2.328 & 2.201 & 11.978 & 1.917 & 1.892 & 7.400 \\
\hline 9-state UKF & 2.084 & 0.286 & 2.341 & 2.211 & 11.955 & 1.907 & 1.905 & 7.428 \\
\hline $\begin{array}{c}\text { 9-state + 6 IMU bias } \\
\text { EKF }\end{array}$ & 1.782 & 0.228 & 1.831 & 2.149 & 12.277 & 1.412 & 1.630 & 6.244 \\
\hline $\begin{array}{c}\text { 9-state + 6 IMU bias } \\
\text { UKF }\end{array}$ & 1.778 & 0.223 & 1.818 & 2.143 & 12.257 & 1.410 & 1.631 & 6.267 \\
\hline
\end{tabular}

* All values are in degrees 
Next, Table 8-3 represents the performance of the formulations when modeling noise directly on the IMU measurement vector.

Table 8-3: Attitude estimation performance between EKF and UKF assuming non-additive noise

\begin{tabular}{|c|c|c|c|c|c|c|c|c|}
\hline \multicolumn{7}{|c|}{ Assuming Non-Additive Noise } \\
\hline $\begin{array}{c}\text { GPS/INS Sensor } \\
\text { Fusion Formulation }\end{array}$ & $\operatorname{mean}(\mathbf{J})$ & $\boldsymbol{\sigma}(\mathbf{J})$ & $\operatorname{mean}\left(\mid \boldsymbol{\phi}_{\text {err }}\right)$ & $\boldsymbol{\sigma}\left(\boldsymbol{\phi}_{\text {err }}\right)$ & $\max \left(\left|\boldsymbol{\phi}_{\text {err }}\right|\right)$ & $\operatorname{mean}\left(\left|\boldsymbol{\theta}_{\text {err }}\right|\right)$ & $\boldsymbol{\sigma}\left(\boldsymbol{\theta}_{\text {err }}\right)$ & $\boldsymbol{m a x}\left(\left|\boldsymbol{\theta}_{\text {err }}\right|\right)$ \\
\hline 3-state EKF & 2.085 & 0.244 & 2.263 & 2.164 & 12.922 & 1.914 & 2.001 & 8.275 \\
\hline 3-state UKF & 2.070 & 0.255 & 2.249 & 2.166 & 12.753 & 1.904 & 1.967 & 10.194 \\
\hline 9-state EKF & 2.094 & 0.294 & 2.368 & 2.176 & 11.822 & 1.954 & 1.923 & 7.526 \\
\hline 9-state UKF & 2.102 & 0.315 & 2.346 & 2.215 & 11.918 & 1.950 & 1.929 & 7.523 \\
\hline $\begin{array}{c}\text { 9-state + 6 IMU bias } \\
\text { EKF }\end{array}$ & 1.774 & 0.218 & 1.818 & 2.136 & 12.105 & 1.411 & 1.623 & 6.254 \\
\hline $\begin{array}{c}\text { 9-state + 6 IMU bias } \\
\text { UKF }\end{array}$ & 1.778 & 0.214 & 1.823 & 2.147 & 12.202 & 1.404 & 1.626 & 6.316 \\
\hline
\end{tabular}

* All values are in degrees

In Tables 8-1 to 8-3, three decimal places were presented to show how the small differences were between the various algorithms. In that all estimates are referenced with respect to the same 'truth', it must be noted that the resolution of the Goodrich ${ }^{\circledR}$ mechanical vertical gyroscope is on the order of one-hundredth of a degree (i.e. pitch $=0.0183^{\circ}$; roll $=0.0275^{\circ}$ ).

In general, regardless of the noise-assumption (additive or non-additive), the 9 -state+6 IMU bias formulation provide the best overall attitude estimation performance, both with respect to the mean and the standard deviation of the performance index over the 23 sets of flight data. The estimation performance is closely followed by the 9-state and 3-state formulations, for both additive and non-additive noise assumptions using either and EKF or UKF. All of the 3-state and 9-state formulations exhibit nearly identical average performance. Also, as indicated in the $2^{\text {nd }}$ column of Table 8-2 and Table 8-3, the formulations that shows better performance also generally has less flight-to-flight performance variation. 
Upon reviewing Tables 8-2 and 8-3, it is apparent that there is essentially no performance difference with respect to the attitude estimation between EKF and UKF with respect to the formulations considered in this study. This is contrary to the claims of a previous study by van der Merwe et. al. based on simulation [31;32], but closely agrees with the findings of others such as Wendel et. al. [41] and Crassidis [40]. This study offers some additional insight because comparisons were drawn with real flight data.

Figure 8.9 illustrates the importance of relying on a diverse library of flight data, when performing a comparison between two nonlinear estimators using experimental data. In particular, the estimation performance of each of the algorithms over 23 flights does exhibit some variation from flight-to-flight. This is exemplified by considering the all of the formulations with non-additive noise, as shown in Figure 8.9.

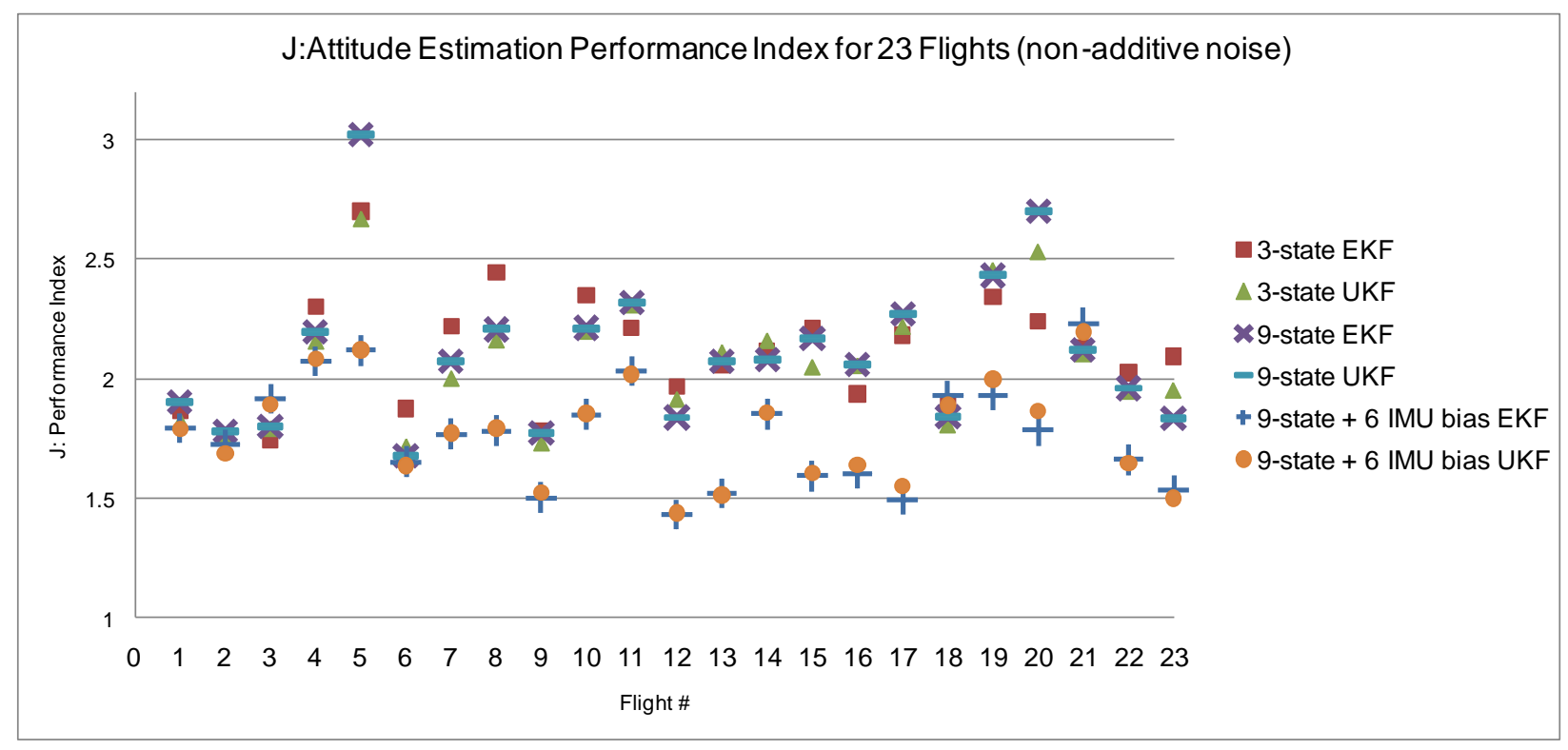

Figure 8.9:Non-Additive Noise GPS/INS attitude estimation performance over 23 for various sensor fusion formulations .

In Figure 8.9 with respect to the 3 -state formulation, notice that if only a smaller subset of flights were used for comparison, it may lead to favoring either EKF of UKF over one another. In addition, while the 9-state and 3-state formulation exhibit close overall average estimation 
performance; there is flight-to-flight variation as to which In order to illustrate the flight-to-flight performance variations. To further illustrate the small differences between EKF and UKF both when assuming noise as additive or non-additive, Figure 8.10 shows the relative performance difference between EKF and UKF for each of the three formulations.

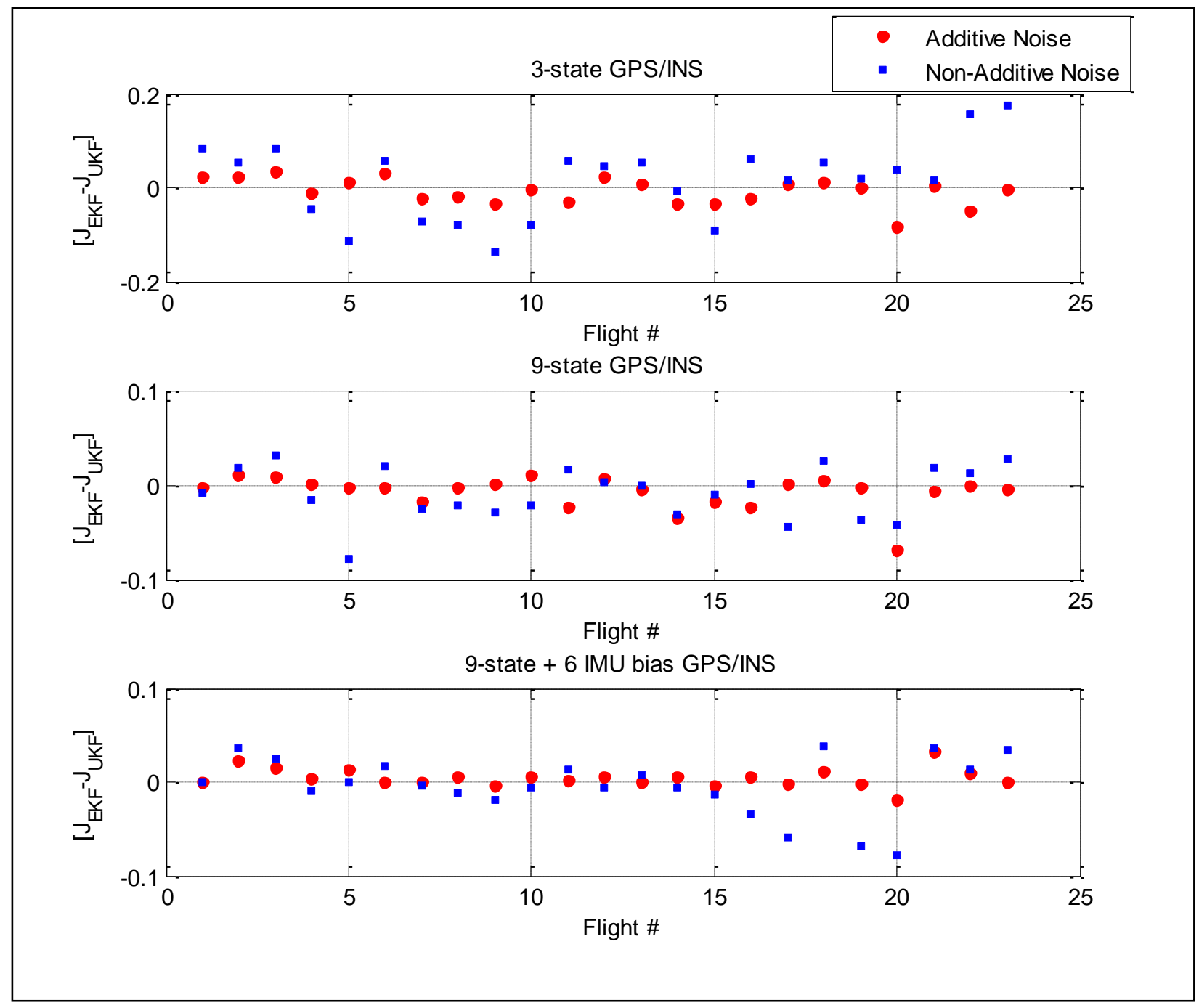

Figure 8.10: Estimation performance variation between EKF and UKF over 23 flights for both types of assumed noise.

In Figure 8.10, the $\mathbf{y}$-axes represent the difference of the EKF estimation performance and UKF performance; so that, a positive value indicates a 'better' UKF and a negative value represent a 'better' EKF. First, it is clear that approximately the same amount of points is distributed above 
and below the y-axis, which is in agreement with the very small differences between the mean estimation performance of EKF and UKF among the formulations. Furthermore, Figure 8.10 shows that the 3-state formulation shows the largest magnitude differences between EKF and UKF, compared to the size of the performance differences between EKF and UKF for the 9-state and 9-state + 6-IMU bias formulations. Another interesting property shown in Figure 8.10 is that there is smaller magnitude variation between the EKF and UKF whenever noise is assumed to be additive.

While Table $8-1$ to $8-3$ and Figures 8.9 to 8.10 provide a comprehensive performance summary between the EKF and UKF for attitude state estimation, it is also interesting to visually compare estimation performance. Because a single plot of all the different sensor fusion estimates against the truth data is impractical, first a visual comparison is conducted that compares the different sensor fusion formulations. That is, over a single example flight ( i.e. Flight \#10 in the library) the 3-state, 9-state, and 9-state + 6 IMU bias GPS/INS sensor fusion estimates are plotted against the vertical gyroscope measurements. For this analysis, the EKF versions that assume additive process-noise are shown, however the same relationships appear when showing the UKF formulations and the non-additive process-noise cases. Figure 8.11 provides an example of pitch estimation performance with respect to the vertical gyroscope. 

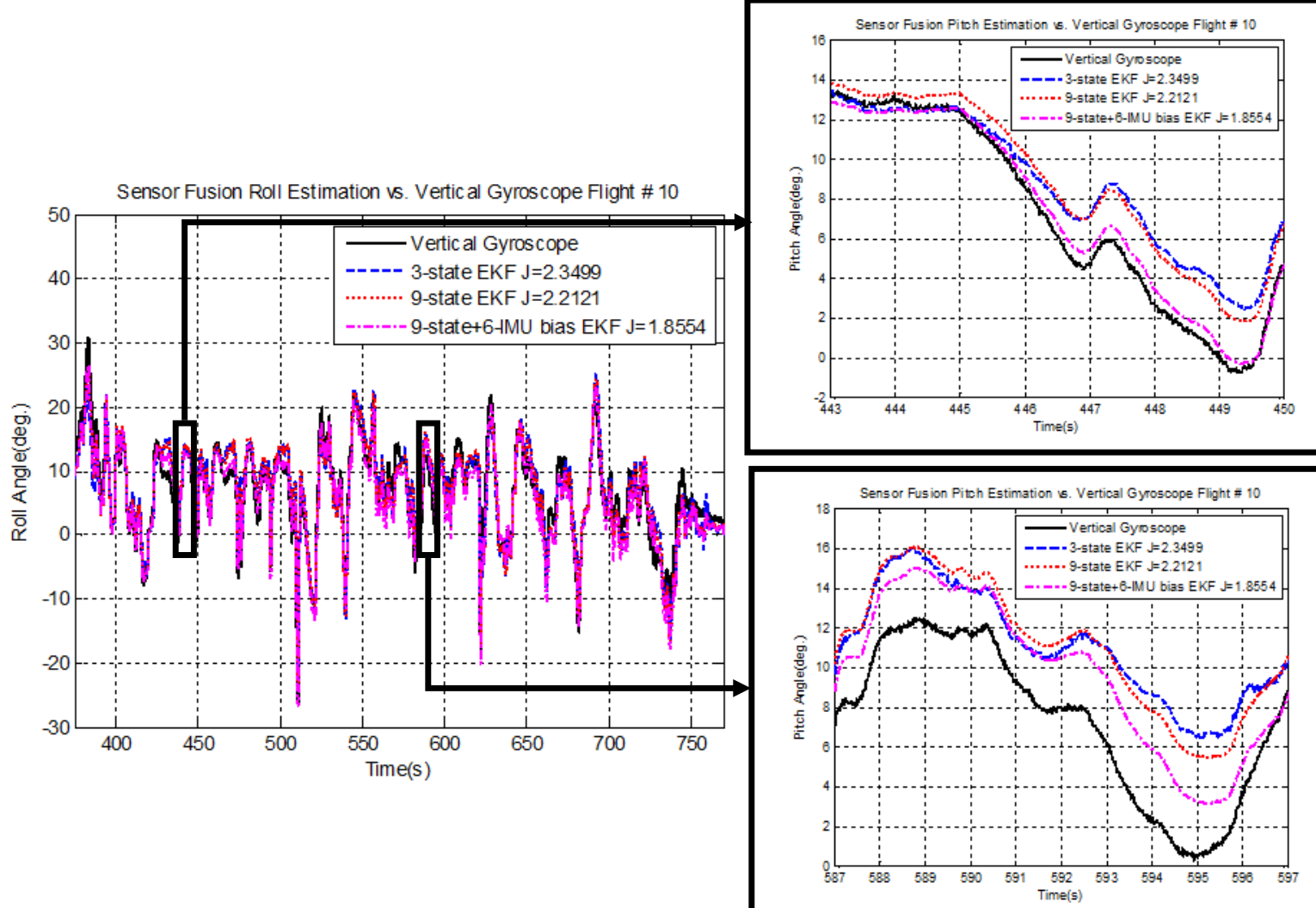

Figure 8.11: Pitch estimation compared with the vertical gyroscope measurement of different GPS/INS sensor fusion formulations using an EKF (assuming additive noise).

In Figure 8.11, the global view on the left shows the overall good agreement with the vertical gyroscope, which is far superior in comparison to the performance shown in Figure 8.2. The two zoomed views show that the bias tracking formulations are closer to the gyro measurement, which is expected given the results shown in Table 8-2. Figure 8.12 provides an example of roll estimation. 


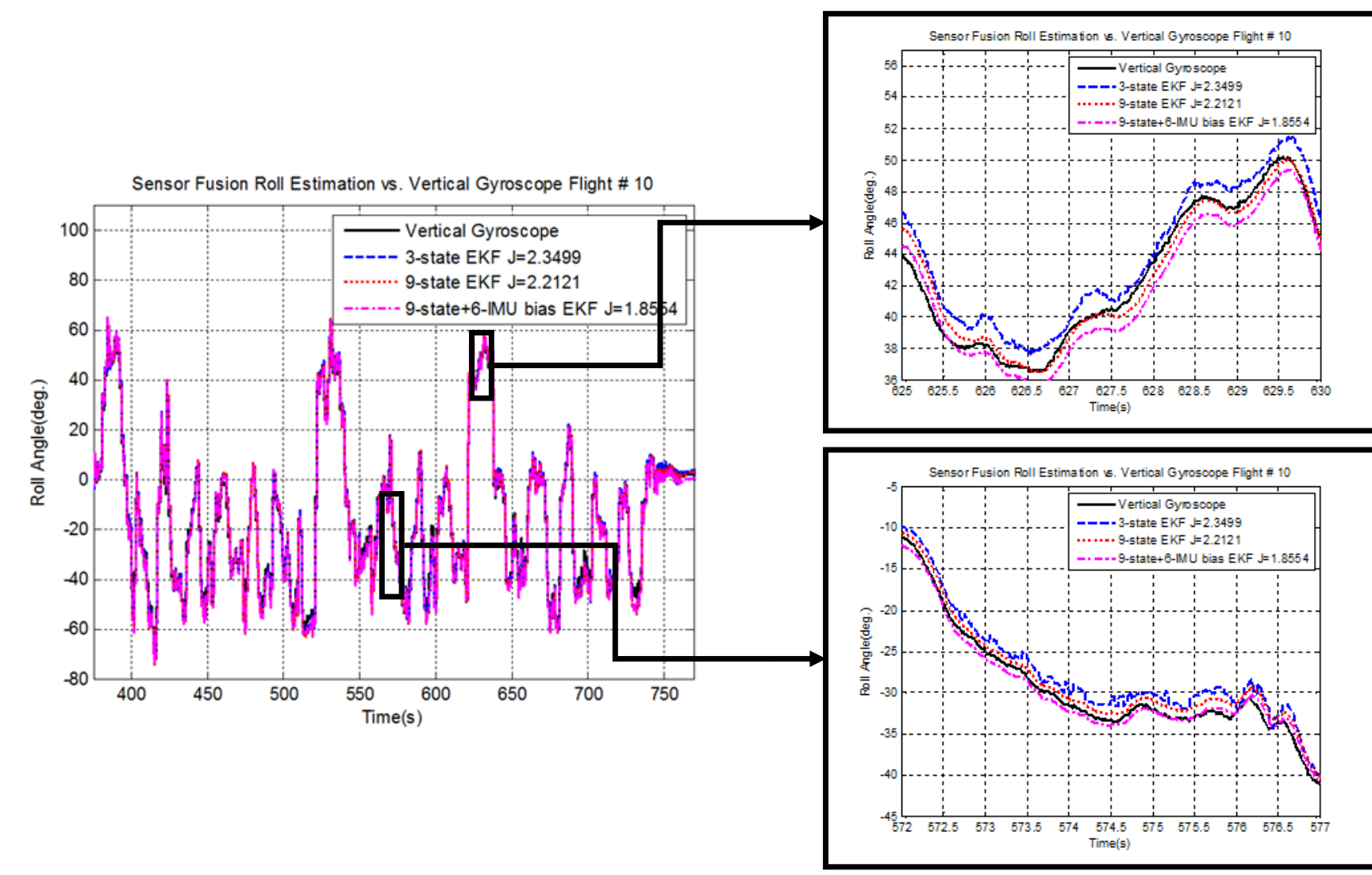

Figure 8.12: Roll estimation compared with the vertical gyroscope measurement of different GPS/INS sensor fusion formulations using an EKF.

Similar trends are shown with respect to roll estimation in Figure 8.12. The global view shows close agreement with the mechanical gyroscope. The zoomed views on the right of the Figures show that the larger order filters appear to exhibit smoother estimated when compared to the 3 state attitude only formulation.

Next, to visually compare the EKF and UKF, they are both plotted against the vertical gyro for all three formulations for the non-additive process noise case. The additive processnoise cases show the same trends. Figures 8.13 and 8.14 provide visual examples of pitch estimates and roll estimates of the EKF and UKF against the vertical gyroscope. 
3-State EKF \& UKF Pitch Estimation vs. Vertical Gyroscope Flight \#10

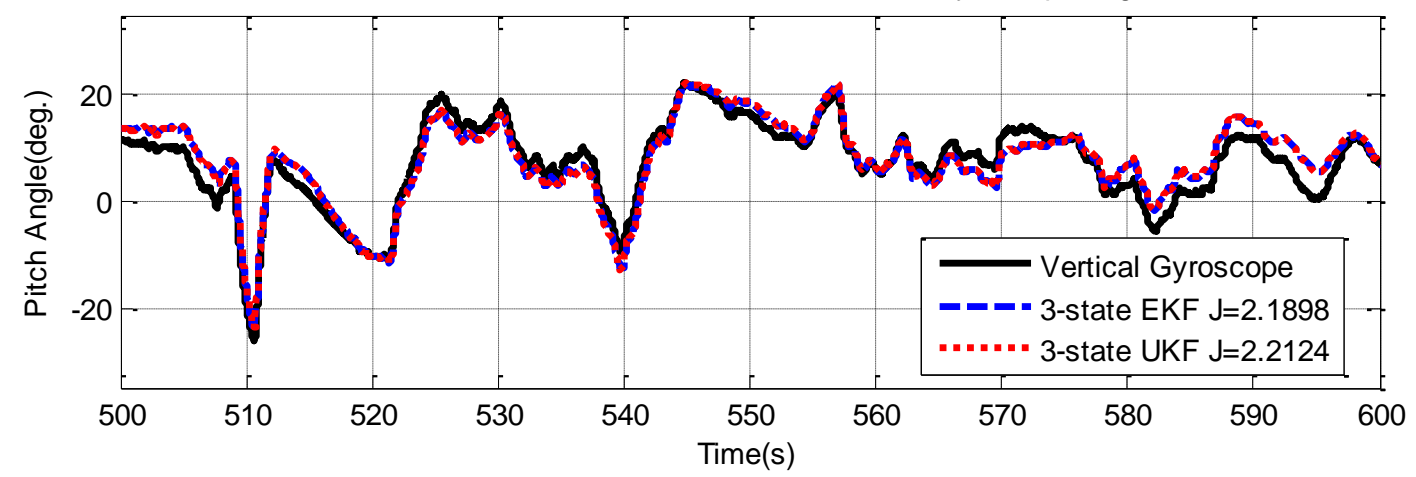

9-State EKF \& UKF Bias Pitch Estimation vs. Vertical Gyroscope Flight \#10



9-State+6 IMU bias EKF \& UKF Pitch Estimation vs. Vertical Gyroscope Flight \#10

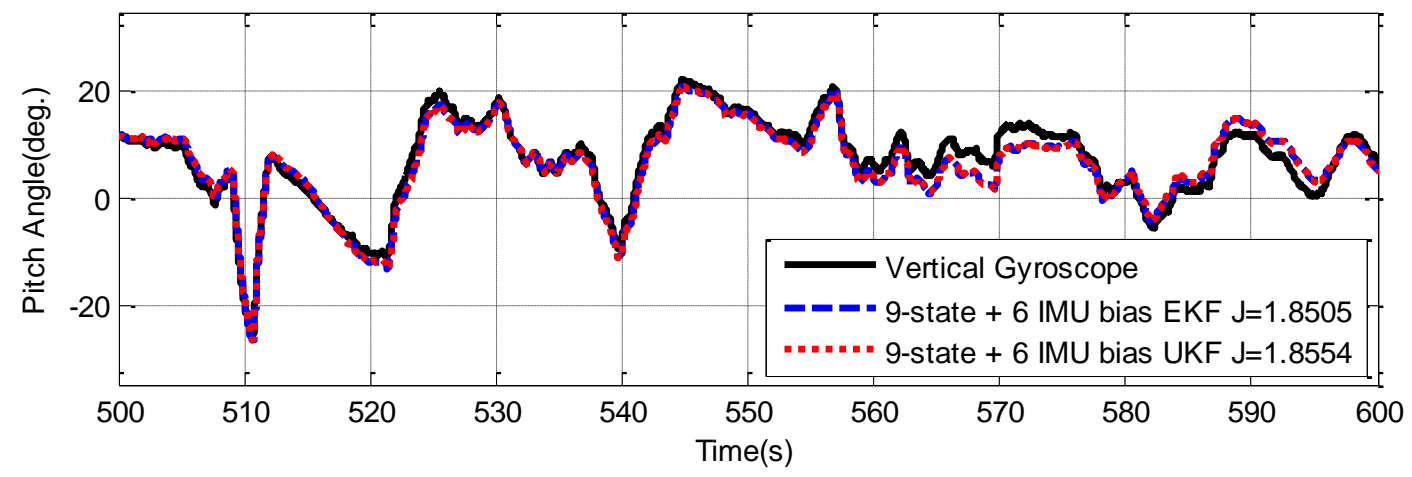

Figure 8.13: Pitch Estimation EKF and UKF comparison for various GPS/INS formulations. 


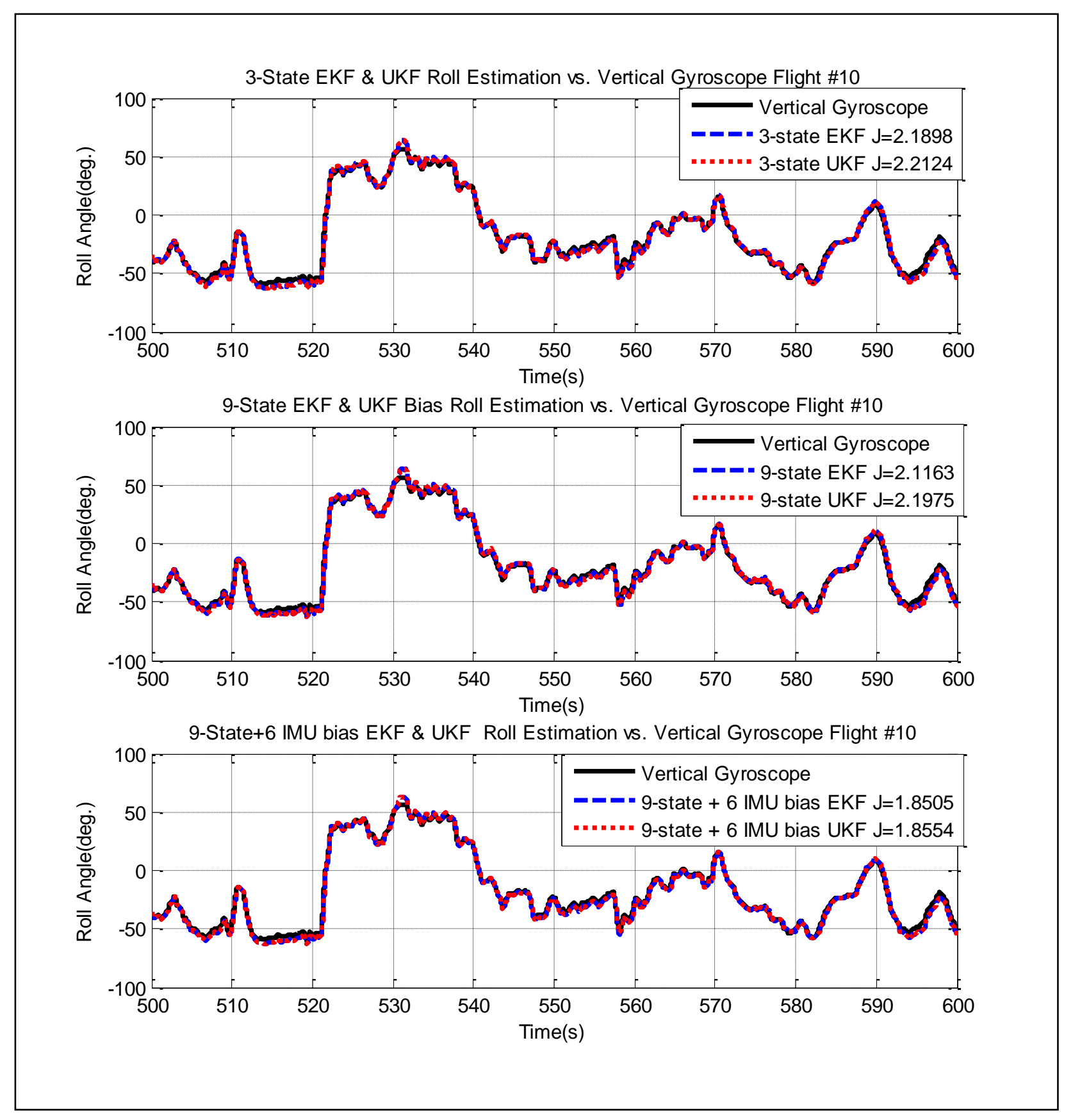

Figure 8.14: Roll Estimation EKF and UKF comparison for various GPS/INS formulations.

Figures 8.13 and 8.14 shows no perceivable differences between the estimations provided by EKF and UKF for each of the three formulations. 


\subsection{EKF and UKF Linearization Procedure Comparison}

Another method used to assess the difference between EKF and UKF in GPS/INS applications was a comparison of the linearization methods is conducted. As discussed in throughout Chapter 5 and during the literature review in Chapter 2 a fundamental difference between EKF and UKF is that EKF relies on an analytical linearization of the nonlinear state transition and observation equations, while UKF transforms random states over a set of sigmapoints statistically. As noted by Lefebvre, Bruyninckx, and De Scheller [34], and Vercauteren and Wang [21] the sigma-point transformations can be viewed as a statistical linearization. That is, a Weighted Least Squares Regression (WLSR) is carried out with the sigma-point, where a linear transition matrix, $A$, and a bias term $c$ are used to approximate the nonlinear function, $f(\mathbf{x})$

$$
f\left(\overline{\mathbf{x}}_{k-1 \mid k-1}\right)=\overline{\mathbf{x}}_{k \mid k-1} \approx A \overline{\mathbf{x}}_{k-1 \mid k-1}+c
$$

This WLSR minimizes the weighted squared error between the true nonlinear predicted states and the linear model that is used to approximate the transition, $A$.

$$
e=f\left(\overline{\mathbf{x}}_{k-1 \mid k-1}\right)-\left(A \overline{\mathbf{x}}_{k-1 \mid k-1}+c\right)
$$

In Equation $8.4 \overline{\mathbf{x}}_{k-1 \mid k-1}$ and $\overline{\mathbf{x}}_{k \mid k-1}$ represent the weighted mean of sigma-points before and after being transformed through the nonlinear function respectively. Specifically, the solution for $A$ and $c$ that minimizes the linearization error $e$ is provided with a standard weighted least square curve fitting method $[21 ; 34]$. 


$$
\begin{aligned}
& A=P_{\mathbf{x}_{k-1 \mid k-1} \mathbf{x}_{k \mid k-1}}\left(P_{\mathbf{x}_{k \mid k-1} \mathbf{x}_{k \mid k-1}}\right)^{-1} \\
& c=\overline{\mathbf{x}}_{k \mid k-1}-A \overline{\mathbf{x}}_{k-1 \mid k-1}
\end{aligned}
$$

Using the insights in $[21 ; 34]$, the difference between UKF and EKF for this specific application was quantified by comparing results of the two linearization processes over a single discrete time-step. Figure 8.15 shows the matrix norms of the attitude sub-matrix of both EKF (analytical linearization) and UKF (WLSR) linearized time-update models (i.e. $A$ ), and the norm of their difference obtained over a single flight. The top plot in Figure 8.15 compares $\left\|A_{k}(\phi, \theta, \psi)\right\|_{2}$ obtained using both the EKF and UKF procedures, and $\left\|A_{k}^{U K F}(\phi, \theta, \psi)-A_{k}^{E K F}(\phi, \theta, \psi)\right\|_{2}$ is shown in the bottom plot. For this example, the 9-state sensor fusion algorithms that assume noise directly on the IMU were used. 


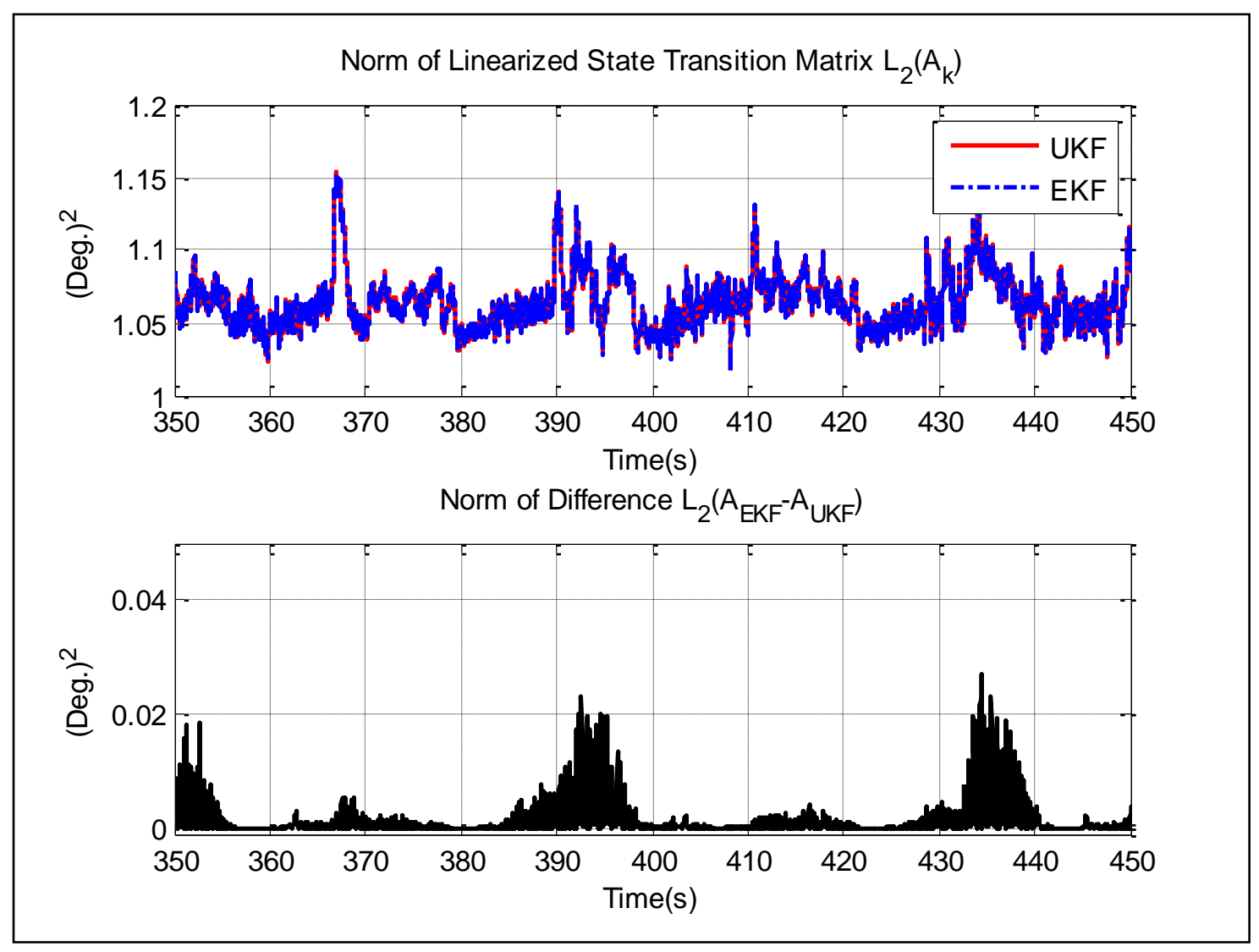

Figure 8.15: EKF and UKF locally linearized attitude models over time.

As indicated in Figure 8-15, for this particular application, the linearization approaches produce very similar linear models. The differences are likely attributed to the additional bias terms $c$ that the WLSR procedure includes to minimize linearization error. While small in magnitude, in order to capture the effect of these bias terms, the single discrete time-step predictions based on the EKF and UKF linearization methods for attitude mean and error covariance are shown in Figure 8.16 and Figure 8.17. 



Figure 8.16: EKF and UKF 1 time step attitude magnitude prediction difference. 


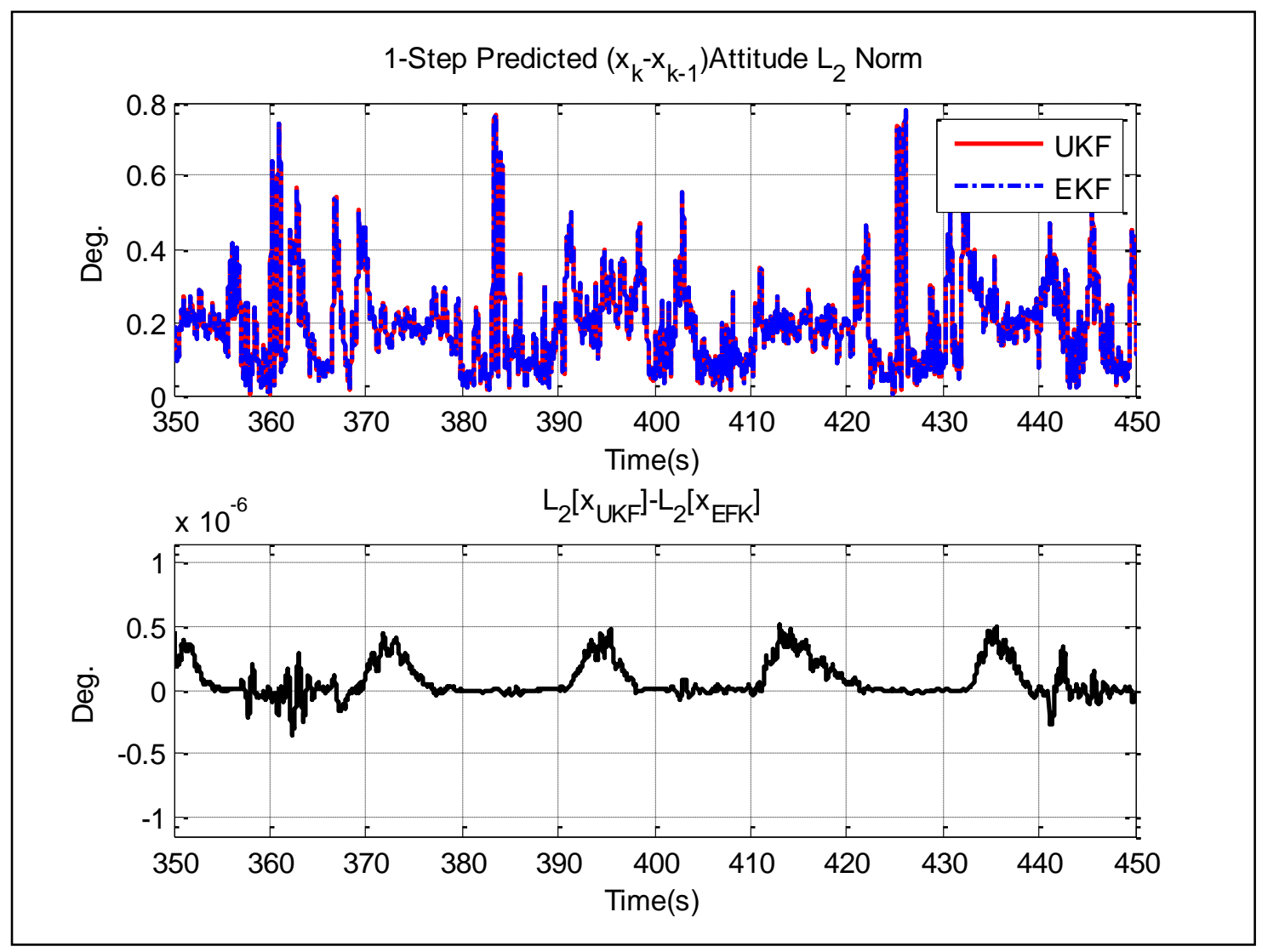

Figure 8.17: EKF and UKF 1 time-step covariance prediction magnitude difference.

As shown in both Figures 8.16 and 8.17, the linearization methods used for EKF and UKF produce models that propagate almost the same mean and covariance estimates over the single time-step.

A clear difference between EKF and UKF is the use of analytical linearization by EKF as compared to the use of statistical linearization [21;34] within UKF. Both the 9-state and 9state+6 IMU bias formulations presented above have nonlinear state transition functions and linear observation functions. Therefore, the EKF and UKF implementations are different only during the prediction step while the standard linear Kalman Filter measurement update procedure [45], is used for both filters. However, in the 3-state formulation, nonlinearity is present in both the prediction and update stages. While minor differences in the two different linearization 
approaches were detectable in the above analysis, this did not contribute of a noticeable performance difference between EKF and UKF.

Another difference that is often attributed to the EKF and UKF is the method for handling the process and measurement noise. Specifically, within UKF, sigma-points are created to augment the state-vector according to known or assumed statistical distributions of sensor noise, which are then directly considered at the sensor-level on sensor outputs and transformed through nonlinear equations in order to model uncertainty. This implicitly (statistically) linearizes around the assumed sensor noise. Instead, the traditional form of the EKF linearizes the nonlinear state transition and observation equations with respect to states and separately with respect to the noise assumptions, in order handle uncertainty. This difference again was shown to have little effect on the estimation performance for this application.

\subsection{Nonlinear Information Filtering vs. Nonlinear Kalman Filtering}

It is known that for linear applications, the IF and $\mathrm{KF}$ are equivalent, however for multiple measurement-updates the IF has the good property that measurement updates are decentralized. When considering that the overall goal of this study is to develop a fault-tolerant algorithm, the property of decentralized measurement-updates is attractive. Because the problem considered it nonlinear, a performance comparison between the nonlinear IF and KF using both Extended and Sigma-Point approaches was conducted in order to determine in any differences arise. For this comparison, the 3-state GPS/INS attitude estimation formulation was chosen. This formulation was chosen because it involves both nonlinear state-transition functions for prediction as well as nonlinear observation functions during the measurement-update procedure. Figure 8.18 and 8.19 show the estimation performance index, $J$, over the 23 flights in the GPS/INS flight library. 


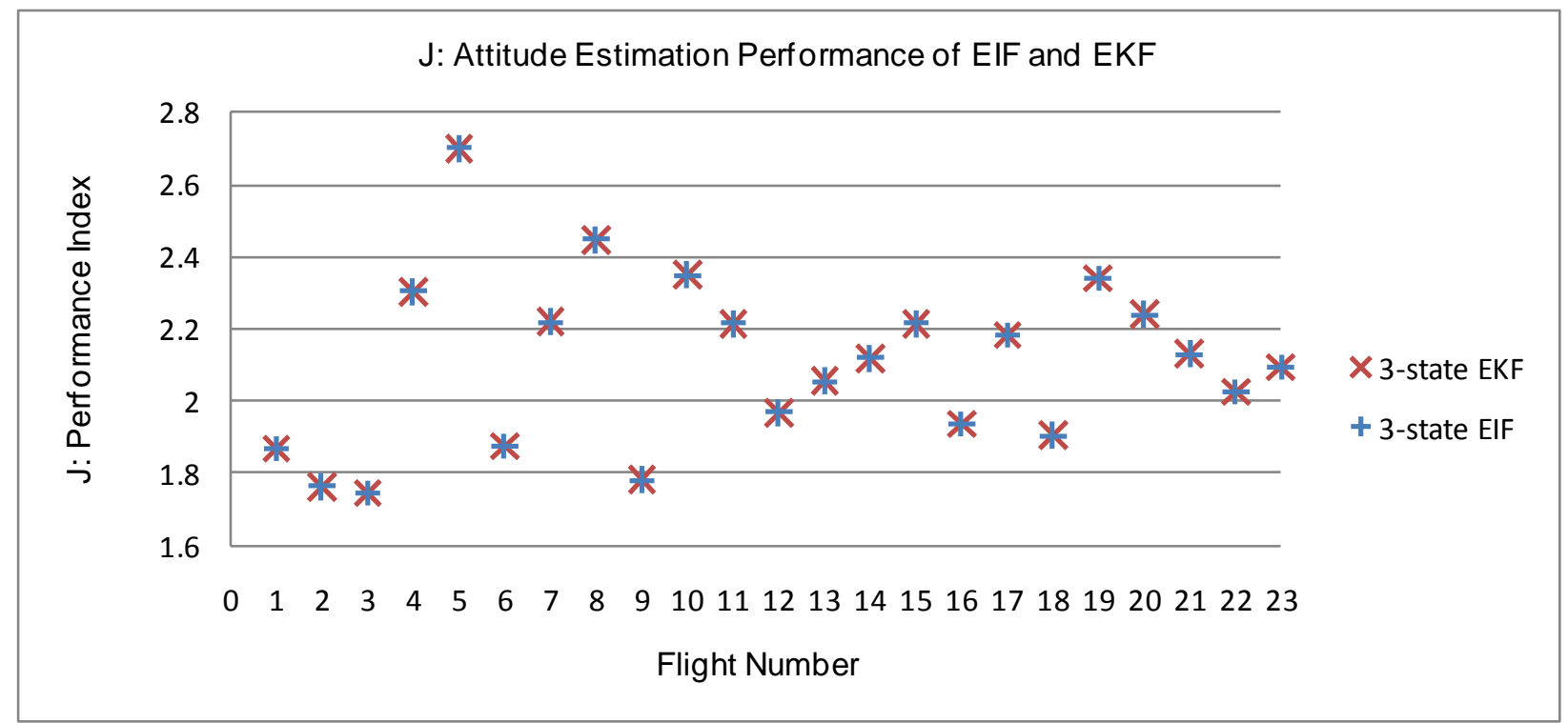

Figure 8.18: Comparison of EKF and EIF over 23 flights.

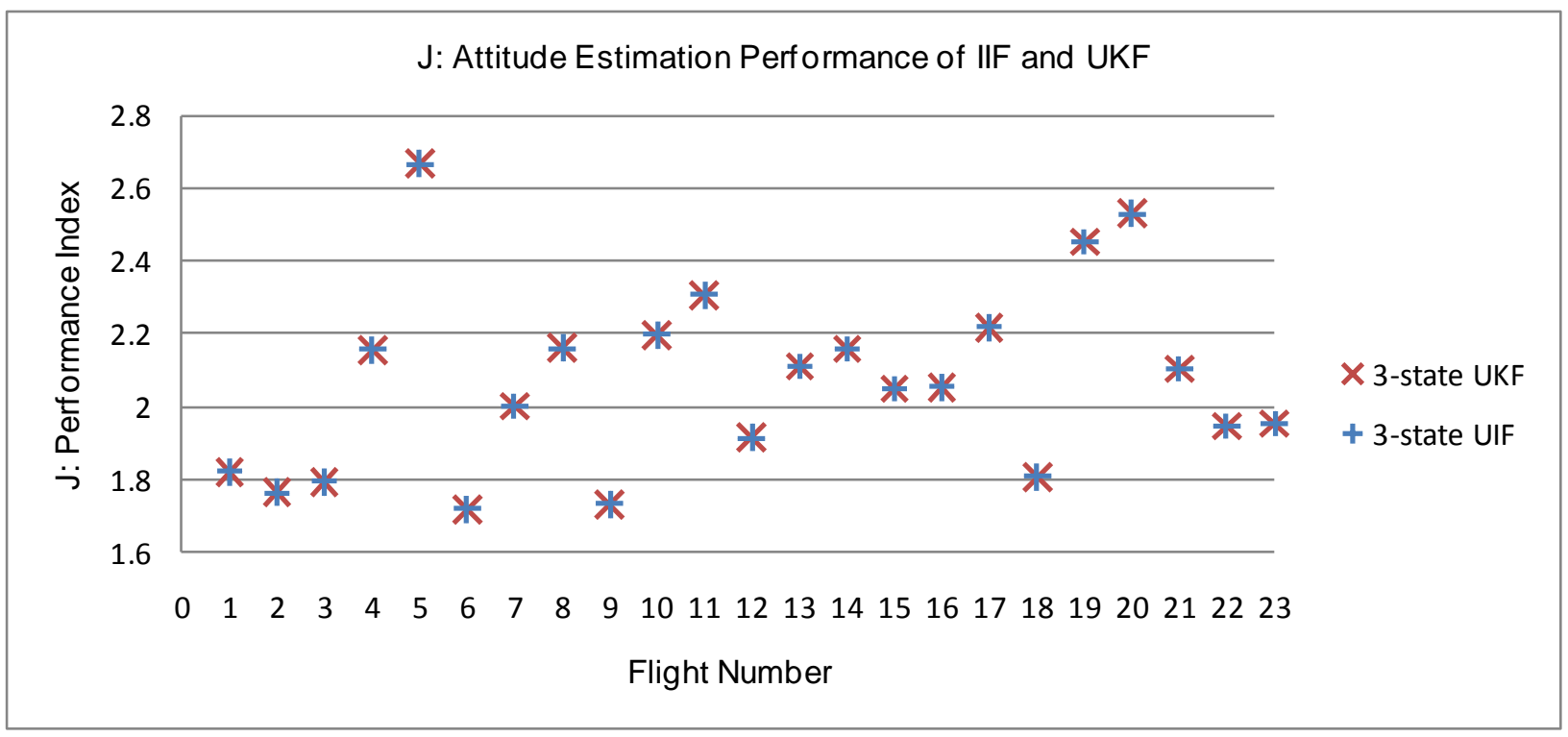

Figure 8.19: Comparison of UKF and EKF over 23 flights.

In Figures 8.18 and 8.19 it is apparent that for the nonlinear forms of the IF are equivalent in terms of performance to the nonlinear forms of the KF over each of the flights in the GPS/INS library. 


\subsection{Position Error Smoothing and On-Line Calibration Position Drift Test}

Because the availability of pitch and roll measurements from a high-quality mechanical gyroscope, the focus of this study is monitoring estimation performance with respect to attitude estimation. However, because the 9-state and 9-state + 6 IMU bias formulations provide estimates of position and velocity, it is also worthwhile to qualitatively assess the position estimates during periods of obvious GPS measurement noise. Figure 8.20 shows some examples of where the low-cost inertial information rejects large jumps in the GPS measurement, and provides a smooth solution.

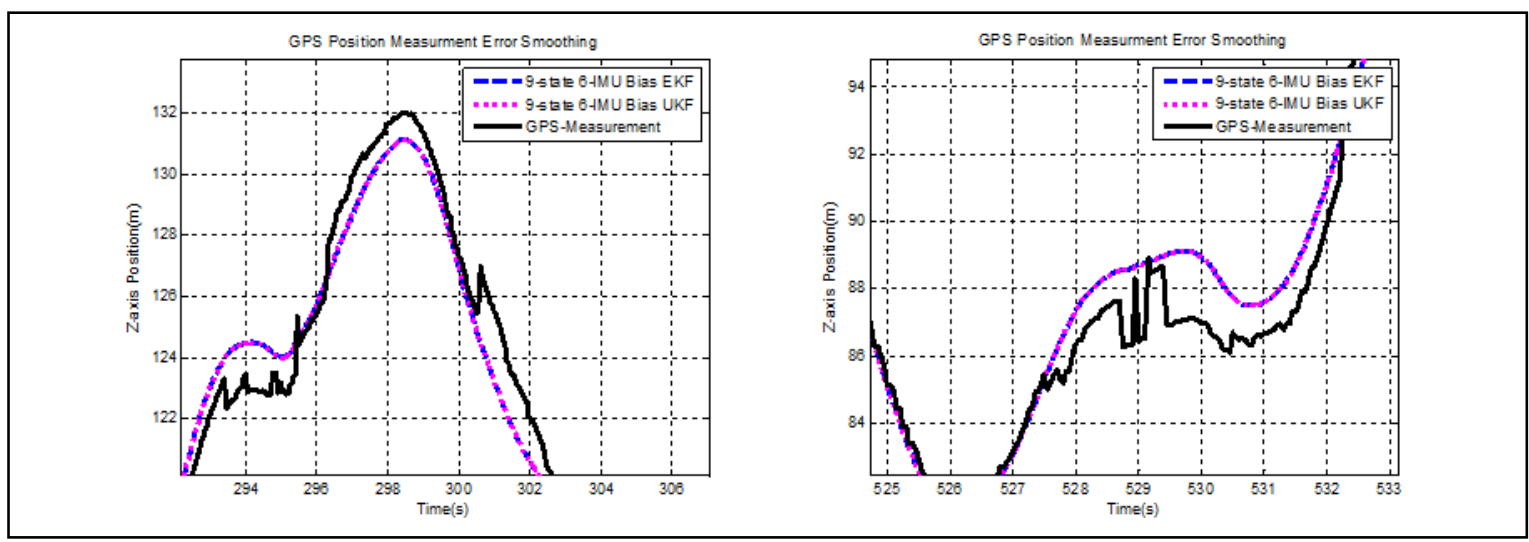

Figure 8.20: GPS Measurement error smoothing with 9-state + 6-IMU bias GPS/INS sensor fusion.

Due to the fact that no independent 'truth' source for position of the SUAV, the extent of this analysis is limited visual interpretation, but in Figure 8.20 is appears that the GPS/INS solution provides a more reliable approximation of position than stand-alone GPS.

Position tracking error of INS dead reckoning monitored in a local navigation frame is not only dependent on the double integration of accelerometer readings, but also is dependent on the time integration of rate-gyroscope that are used to provide the attitude necessary to form a DCM that related the orientation of the accelerometers in the body-axis frame to the local 
navigation frame. Therefore, position tracking error is often a metric used to evaluate the performance on an INS calibration procedure [94; 92]. Furthermore, in the above performance analysis of the different sensor fusion algorithms that was evaluated with respect to attitude estimation, it was shown that the 9-state + 6-IMU bias tracking filters provided enhancements with respect to attitude estimation. In order to further demonstrate the performance of the 9-state + 6-IMU GPS/INS formulation, Figure 8.21, represents the position tracking error with respect to a baseline GPS/INS reference trajectory of the bias tracking filter vs. the tracking error of a state only estimation filter.

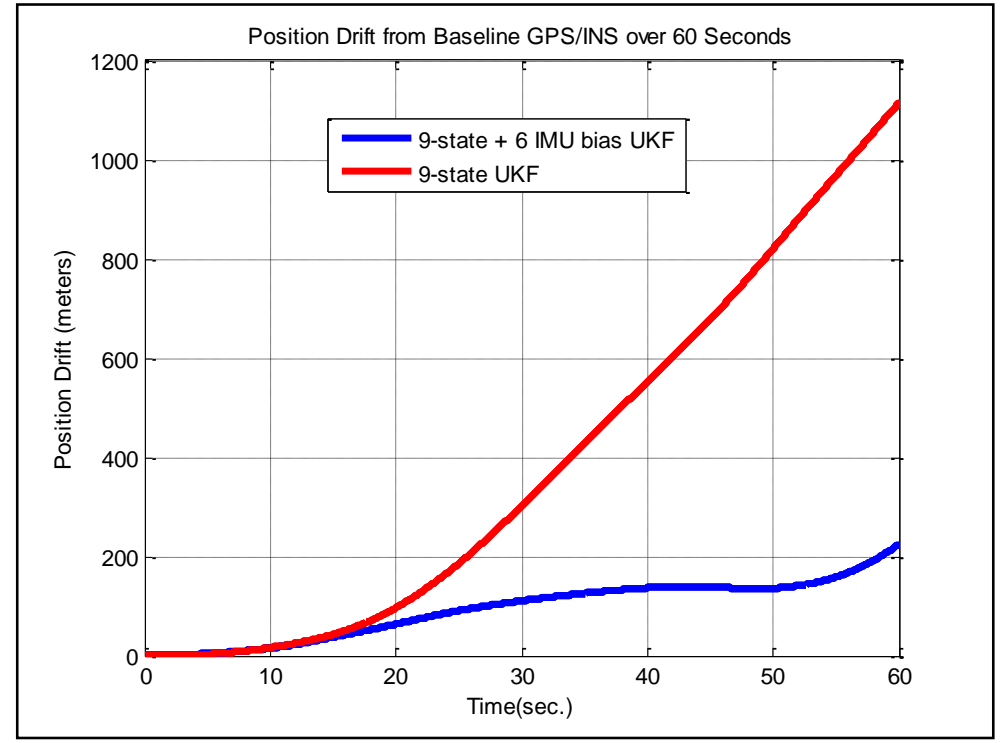

Figure 8.21: Position drift of the on-line IMU calibration filter during a 1 minute GPS outage.

As shown in Figure 8.21, the position drift during a 1-minute GPS outage of the on-line calibration filter was reduced from 1,114 meters to 223.6 meters for a reduction of over 5 times. While Figure 8.21 shows and example of a single trajectory, these results were typical among all the bias tracking filters both with additive and non-additive noise. 


\section{Chapter 9 : GPS/INS/Magnetometer Sensor Fusion and Calibration}

Some of the results described in this Chapter are summarized and presented in a journal article submission [98], and a conference article [99].

Within in this study, an extensive comparison of EKF and UKF when considering different noise assumptions was carried out for GPS/INS over a diverse library of flight data, the results of which are presented in Chapter 8. In addition, the equivalent estimation performance of nonlinear Ifs and KFs for GPS/INS attitude estimation was also demonstrated. Throughout this Chapter, GPS/INS/Magnetometer fusion formulations are considered. For this analysis, a more limited amount of SUAV flight data was available. Specifically, three sets of flight data were collected that included GPS/INS and Magnetometer information, and one of them is used to calibrate the magnetometers. As indicated in Chapter 8, the flight-to-flight variations between the EKF and UKF performance are such that basing comparisons between the two types of estimators with only limited number flights could be misleading. Moreover, Chapter 8 indicated that EKF and UKF provide essentially the same estimation performance for GPS/INS attitude estimation. Therefore, in the remaining chapters of this dissertation the UKF was utilized with noise assumed directly on sensor readings, and comparisons between EKF and UKF are no longer carried out. The remaining focus of the dissertation was to include magnetometers Chapter 9 and the design of a fault-tolerant attitude estimation algorithm Chapter 10.

\subsection{GPS/INS/Magnetometer Sensor Fusion}

For all the GPS/INS/Magnetometer formulations it is assumed that all sources of information are available at $50 \mathrm{~Hz}$. Therefore because the GPS was recorded at $20 \mathrm{~Hz}$, it was interpolated. This assumption was necessary to implement the fault-tolerant algorithm presented 
in Chapter 10. Novatel's latest OEM4 GPS receivers are available with a $50 \mathrm{~Hz}$ update rate, which have recently been integrated in the Gen-V avionics system, however, when flight data was collected for this study, $20 \mathrm{~Hz}$ was the maximum available GPS update rate.

\subsection{Off-line and On-Line Low-Cost Magnetometer Calibration}

The magnetometer calibration procedure that uses a GPS/INS attitude estimates that was outlined in Section 7.4 was carried out using the attitude solution from a 3-state +6 IMU bias GPS/INS UKF, and the resulting calibration model was determined as given in Equation (9.1).

$$
\begin{aligned}
A_{M} & =\left[\begin{array}{ccc}
0.9788 & 0.0248 & 0.207 \\
-0.0248 & 0.991 & 0.0155 \\
-0.0193 & -0.0155 & 1.0274
\end{array}\right] \\
B_{M} & =\left[\begin{array}{lll}
28.54 & -62.36 & -74.53
\end{array}\right]^{T}
\end{aligned}
$$

In Equation (9.1), $A_{M}$ is the multiplicative result of the Scale factor matrix and rotation matrix. To further calibrate the magnetometers, the 3-state + 9-bias GPS/INS/Magnetometer formulation was used, as shown in Chapter 7. Figure 9.1 illustrate the effect of magnetometer calibration on roll and pitch estimation. 

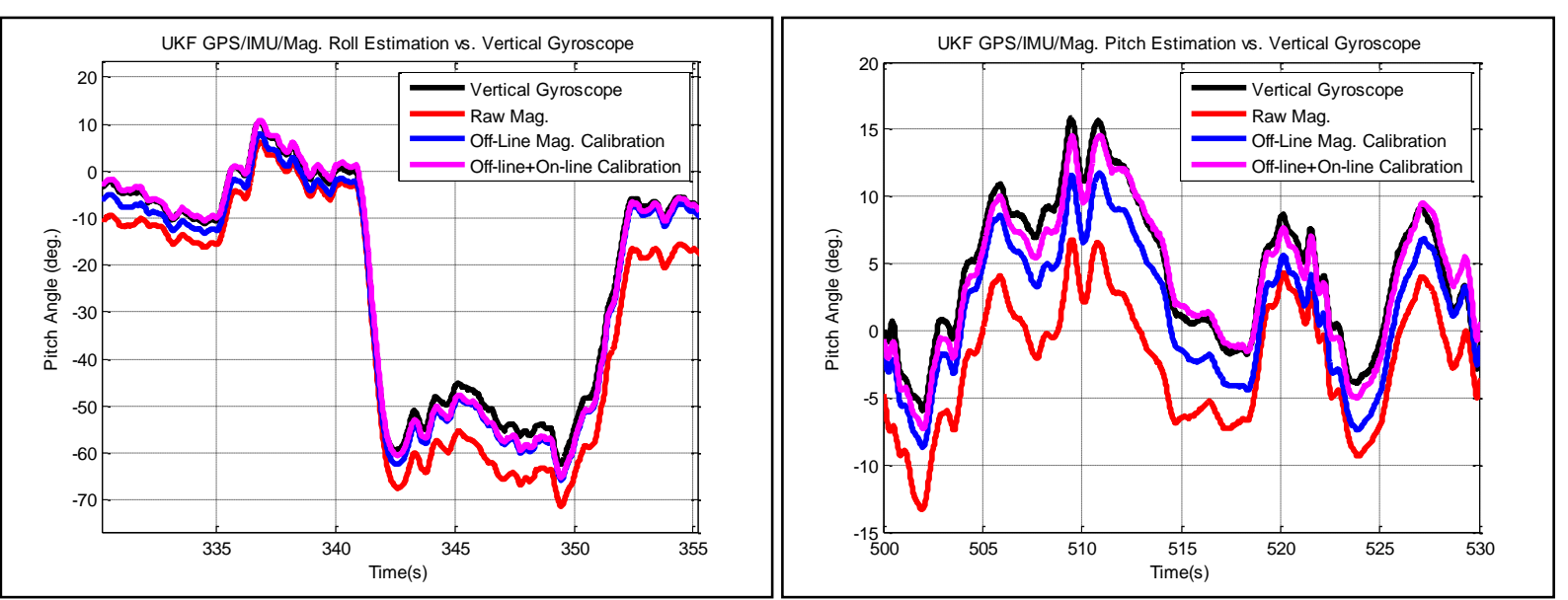

Figure 9.1: Effect of magnetometer calibration on attitude Estimation, Off-Line and Off-Line with Off-Line

Figure 9.1 clearly shows that the off-line magnetometer calibration procedure that relied on GPS/INS improves the attitude estimates. In addition, it further shows improvements in performance when including on-line sensor fusion based calibration.

Using the two remaining sets of flight data that were not used for magnetometer calibration that had GPS, IMU, and magnetometer information and the vertical gyroscope available for a reference 'truth', the estimation performances of the various sensor fusion attitude estimation algorithms are summarized in the next section.

\subsection{GPS/IMU/Magnetometer Sensor Fusion Performance Comparison}

Table 9-1 summarizes the estimation performance of a variety of sensor fusion algorithms that used GPS, IMU, and or magnetometers for attitude estimation, and are averaged over two flights. 
Table 9-1: GPS/INS/Magnetometers attitude estimation performance summary.

\begin{tabular}{|c|c|c|c|c|c|c|c|}
\hline $\begin{array}{c}\text { Attitude Sensor Fusion } \\
\text { Formulation }\end{array}$ & $\operatorname{mean}(J)$ & $\operatorname{mean}\left(\left|\phi_{\text {err }}\right|\right)$ & $\sigma\left(\phi_{\text {err }}\right)$ & $\max \left(\left|\phi_{\mathrm{err}}\right|\right)$ & $\operatorname{mean}\left(\left|\theta_{\mathrm{err}}\right|\right)$ & $\sigma\left(\theta_{\text {err }}\right)$ & $\max \left(\left|\theta_{\text {err }}\right|\right)$ \\
\hline 3-state GPS/INS UKF & 1.977 & 2.990 & 1.671 & 8.927 & 1.590 & 1.865 & 5.365 \\
\hline $\begin{array}{c}\text { 3-state GPS/INS +6 Bias IMU } \\
\text { UKF }\end{array}$ & 1.582 & 1.516 & 1.524 & 6.650 & 1.558 & 1.700 & 5.607 \\
\hline $\begin{array}{c}\text { 2-state GPS/Mag. UKF (Off-Line } \\
\text { Magnetometer Calibration w/ } \\
\text { GPS/INS) }\end{array}$ & 2.069 & 1.726 & 2.008 & 24.117 & 2.315 & 2.195 & 19.382 \\
\hline 3-state GPS/Accel./Mag. UKF & 1.891 & 1.547 & 1.933 & 19.303 & 1.754 & 2.170 & 16.714 \\
\hline $\begin{array}{c}\text { 3-state IMU/Mag. UKF (Off-Line } \\
\text { Mag. Calibration Based on } \\
\text { GPS/INS) } \\
\end{array}$ & 21.943 & 15.809 & 19.588 & 50.089 & 22.989 & 27.688 & 47.052 \\
\hline $\begin{array}{c}\text { 3-state GPS/IMU/Mag. UKF ( } \\
\text { Raw Mag.) }\end{array}$ & 4.317 & 8.061 & 3.219 & 15.617 & 5.276 & 2.279 & 11.953 \\
\hline $\begin{array}{l}\text { 3-state GPS/IMU/Mag. UKF } \\
\text { (Off-Line Mag. Calibration) }\end{array}$ & 1.962 & 2.026 & 1.452 & 7.709 & 2.415 & 2.127 & 6.134 \\
\hline $\begin{array}{c}\text { 3-state GPS/IMU/Mag. UIF (Off- } \\
\text { Line Mag. Calibration) }\end{array}$ & 1.962 & 2.026 & 1.452 & 7.709 & 2.415 & 2.127 & 6.134 \\
\hline $\begin{array}{c}\text { 3-state GPS/IMU/Mag. UIF (Off- } \\
\text { Line Mag. +On-Line Mag./INS } \\
\text { Calibration) }\end{array}$ & 1.616 & 1.598 & 1.697 & 6.976 & 1.417 & 1.679 & 6.286 \\
\hline
\end{tabular}

* All values are reported in degrees

Table 9-1 shows several formulations including off-line magnetometer calibration, on-line sensor calibration, and formulations that use only a subset of the sources of information. Upon in inspection, Table 9-1 reaffirms the trend shown in Figure 9.1. That is, the off-line magnetometer calibration procedure that utilizes GPS/INS attitude to calibrate the magnetometers improved the estimation performance in comparison to using the raw magnetometer readings (compare row 6 with row 7). Furthermore, once on-line bias tracking was included, the estimation performance of GPS/IMU/Mag. further increased (row 9). However, upon comparing rows 2 and 9, it is apparent the GPS/INS attitude estimation with on-line bias tracking provides slightly better performance than GPS/INS/Mag. with bias tracking. However, the use of magnetometers with GPS or GPS/Accelerometers and no rate gyroscopes provides a good attitude estimation solution. The good performance of these two formulations is particularly interesting, because they relied on a prediction step based on random walk, and did not use rate gyroscopes. This 
success was not extended to the use of only of only rate gyros and magnetometers (row 5), which does not produce very good attitude estimates. A comparison of 3-state GPS/INS, GPS/Mag., and INS/Mag. sensor fusion formulations is shown in Figure 9.2.
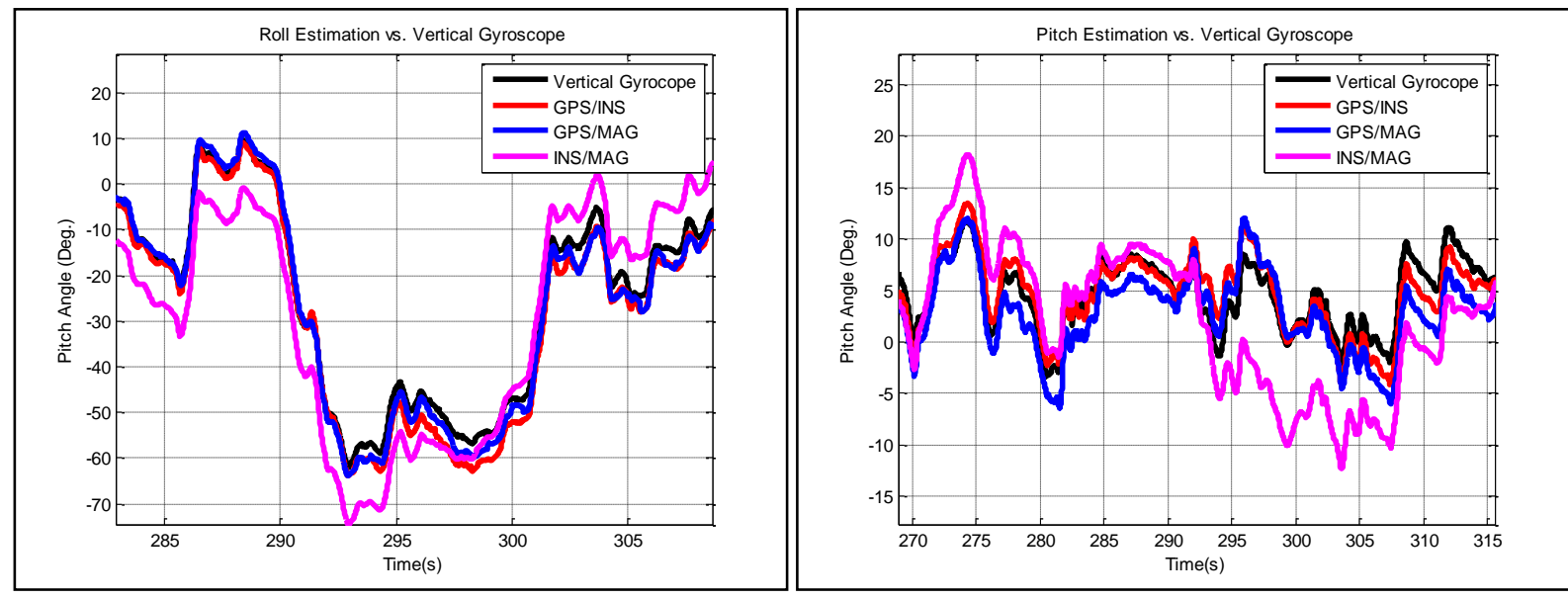

Figure 9.2: Comparison of 3-state GPS/INS, GPS/Mag., and INS/Mag. sensor fusion.

As indicated in Figure 9.2, the GPS/INS and GPS/Mag. both closely follow the vertical gyroscope; however, the INS/Mag. formulation exhibits large errors. This formulation was still included because it may prove useful for short periods of time in the event of a sensor failure. In addition, the INS/Mag. formulation may improve if the Magnetometer and rate gyroscopes are calibrated with on-line bias tracking. These issues are explored in the next Chapter, which is focused on fault-tolerant sensor fusion. Finally, another property indicated is Table 9-1, is that just as the case for 3-state GPS/INS attitude estimation, the UIF and UKF produced the same average estimation error with respect to the vertical gyro (row 7 and row 8). 


\section{Chapter 10 : Fault-Tolerant Attitude Estimation}

Some of the results described in this Chapter are summarized and presented in a journal article submission [98].

\subsection{Redundancy and the Information Filter for Fault Accommodation}

The final phase of this study considers the design of a fault-tolerant attitude estimation algorithm that is built upon on the foundation laid by the nominal condition sensor fusion comparisons presented Chapter 8, and the improved performance accuracy attributed to including magnetometers and on-line sensor calibration as presented in Chapters 8 and 9. Specifically, a fault-tolerant attitude estimation algorithm is derived based on the GPS/INS/Magnetometer attitude estimation formulation outlined in Section 7.2.3, where the 3state attitude estimates are enhanced to include the tracking to time-varying biases of each of the nine low-cost sensors fused ( 3 rate gyroscopes, 3 accelerometer, and 3 magnetometers). The goal of this algorithm is to exhibit high-performing estimation accuracy during sensor failures and to lose performance gracefully during long duration sensor failures. The types of failures accommodated include both 'hard' failures which are defined as occurring quickly and having a large magnitude, as well as 'soft' failures that grow slowly over time and have a smaller magnitude.

The choice of the GPS/INS/Magnetometer formulation was natural due to the fact that if any two of the three partially redundant information sources (GPS/Accelerometers, Rate Gyroscopes, Magnetometers) fused together provides a sensor fusion based attitude estimation solution alone. Additionally, under nominal sensor conditions, the GPS/INS/Magnetometer fusion provides good estimation performance. For the design of a fault-tolerant attitude estimation algorithm, the partially redundancy of the attitude information sources is exploited, and at high-level, the algorithm's FDI procedure can be viewed of as form of implementing a 
voting procedure, where two stages of FDI are utilized to handle failures. The details of the two forms of FDI are described in the next two sections of this chapter.

Both for the case of GPS/INS and GPS/INS/Mag. the KF and IF were shown to provide the same estimates, and because the measurement-update procedure of the IF consists of a trivial sum of information, a UIF was chosen as the nonlinear estimator in design of the fault-tolerant attitude estimation algorithm. Specifically, because the UIF decentralizes the measurementupdate procedure for each source of information (from $j=1$ to $N$ ), and then combines information with a summation,

$$
\begin{aligned}
& i_{k \mid k}=i_{k \mid k-1}+\sum_{j=1}^{N} i_{j, k} \\
& I_{k \mid k}=I_{k \mid k-1}+\sum_{j=1}^{N} I_{j, k}
\end{aligned}
$$

a failure accommodation procedure is accomplished by removing a source of information that has been detected faulty.

Since the UIF provides an easy mechanism for failure accommodation, the remaining hurdle that was needed to implement a fault-tolerant attitude estimation algorithm was the ability to detect sensor failures (i.e. FDI). In this study, FDI of substantial failures is accomplished in two phases. First, FDI is accomplished by isolating the information sources, and representing the statistical agreement between the isolated solutions. Second, FDI of smaller failures is accomplished by monitoring the change of the sensor bias estimates. Information isolation and both types of FDI used are described in the next sections of this Chapter. 


\subsection{Information Isolation}

In the 3-state GPS/INS/Magnetometer sensor fusion formulation, three sources of information are used to estimate attitude: namely, rate gyroscopes, the Earth's gravity vector, and the Earth's magnetic field vector. In the specific formulation presented, the rate gyroscopes are used to predict attitude, or in terms of the nonlinear $\mathrm{KF}$, provide the 'a priori' estimate of mean and error-covariance. Then, both the Earth's gravity vector and magnetic field vector are referenced during measurement-update procedures, to obtain the 'a posteriori' estimates of the sate vector and error-covariance. Where gravity is referenced by using the information from the tri-axial accelerometers and GPS velocity measurements, and the magnetic field is referenced using the information from magnetometers and WMM values. In this scenario, some of the sensors are used for prediction, and some information is used measurement-update, as shown in Figure 10.1.

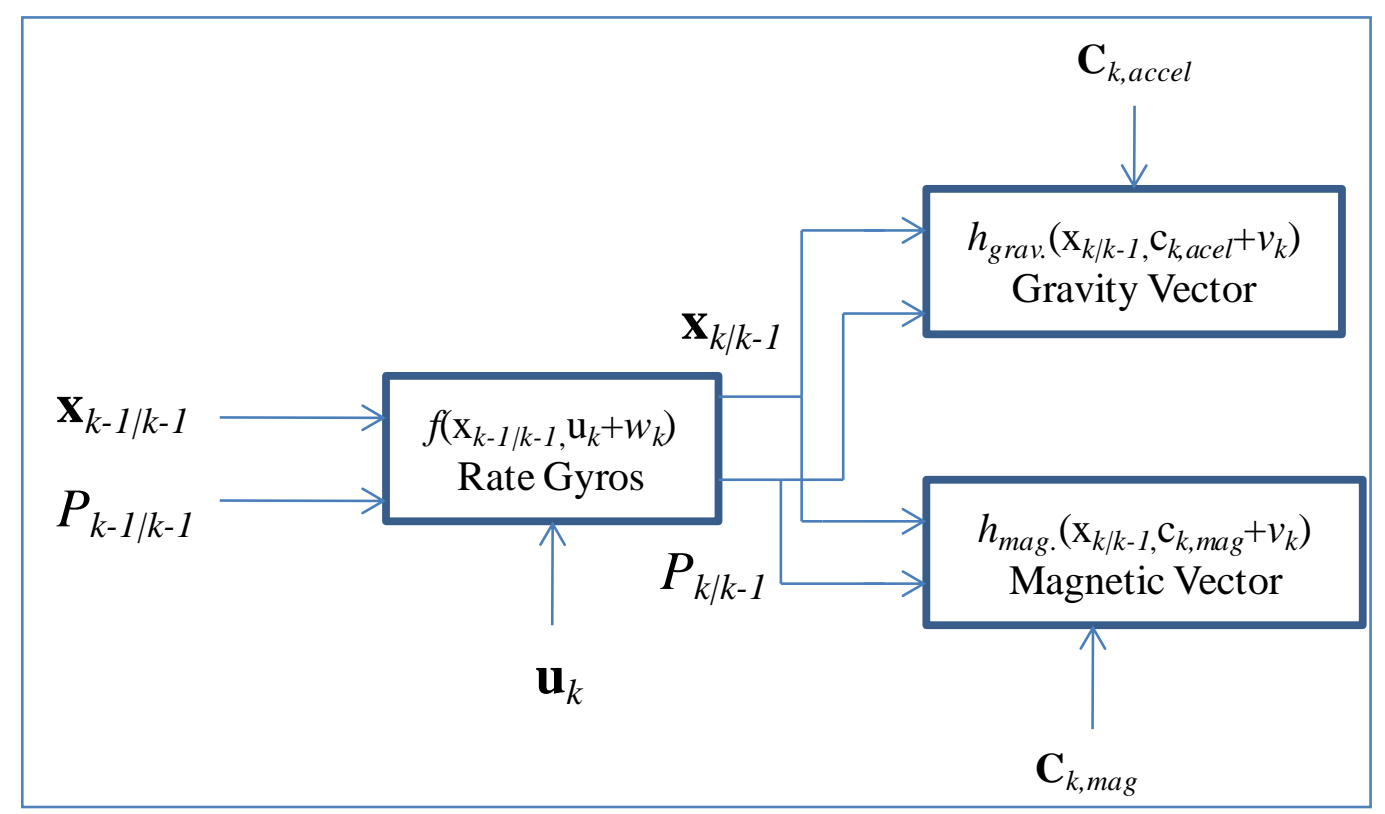

Figure 10.1: GPS/INS/Magnetometer sensor fusion block diagram. 
Using some information for prediction and some for measurement-update provides a straightforward solution whenever all the sensor outputs are exhibiting their nominal level on uncertainty (i.e. close to the $Q$ and $R$ assumptions). However, when considering the implementation of FDIA, for this particular scenario, a difficulty arises when trying to detect a sensor failure that occurs during the prediction step. In particular, if the 'a priori' attitude from the rate gyroscopes is fused either with the GPS/acceleration information or magnetometer information it is difficult to determine whether a sensor failure occurred on the rate sensors or the sensors used for measurement-update. To avoid this problem, it is imperative to isolate information sources for the purposes of FDI.

In order to isolate the three information sources to conduct FDI, the random walk attitude update procedure was used. In this approach, the assumption is made that that the priori' attitude is equal to the 'a posteriori' with an increase level on uncertainty.

$$
\dot{\mathbf{x}}=\mathbf{0}+w_{r w}
$$

The prediction offered by Equation (10.3) proved successful in the GPS/Magnetometer and GPS/Accelerometer/Magnetometer formulations discussed in Chapter 9. In this case, the estimation filter performs a correction based on the measurement-update only. Using Equation 10.3 it is them possible to isolate the three sources of information, as depicted on Figure 10.2 


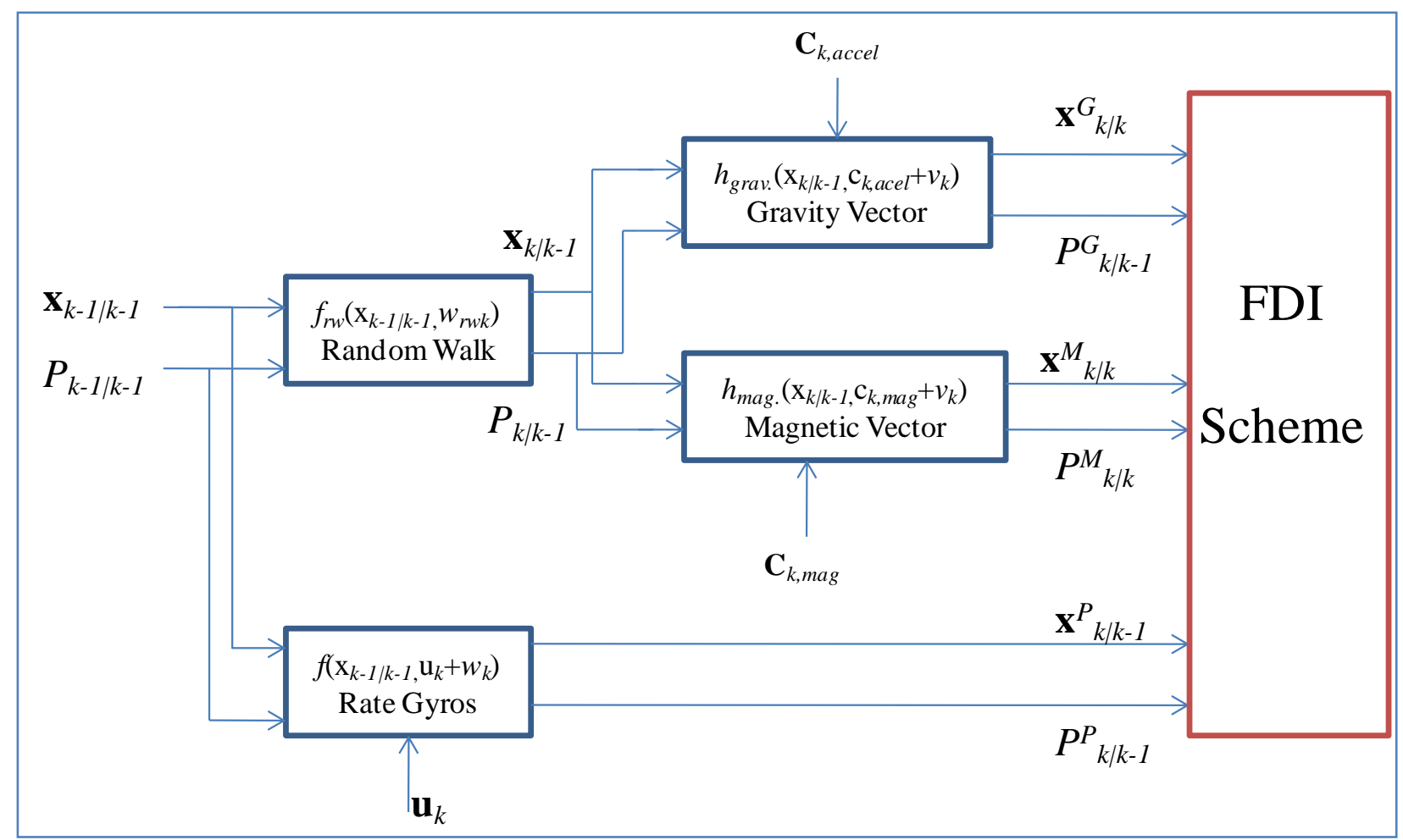

Figure 10.2: Sensor information isolation for FDI.

With the information from the rate gyroscopes (denoted with superscript $P$ ), gravity information (denoted with superscript $G$ ), and magnetometer information (denoted with superscript $M$ ), it is possible to design an FDI scheme. It is important to mention that in the case that the FDI scheme determines that all three sources are nominal; it is desirable to use the approach depicted in Figure 10.1. Therefore, Figure 10.2 only represents the mechanism used to isolate the sensors for FDI, and then information is summed using Equations (10.1) and (10.2) based on the selection of the FDI procedure. 


\section{3 'Virtual Force-Field' Based Failure Detection and Identification}

Using the three isolated information sources, as described in the previous section, the first form of FDI considered in this study is based cross-checking, or voting [98]. This approach I rooted on multivariate normal (MVN) statistical hypothesis testing. More precisely, a $\chi^{2}$,'ChiSquare', statistical hypothesis test $[100 ; 54 ; 56 ; 59 ; 60 ; 101]$ is used to evaluate the agreement between estimates of the state vector and error-covariance that are calculated using different information sources.

First, three sets are defined, $\left(\mathbf{S}_{k}, \mathbf{E}_{k}, I_{k}\right)$, where $\mathbf{S}_{k}$ is a set of $N$ sensor information sources used to calculate estimates, $\mathbf{E}_{k}$ is a set of $N$ state estimates each only based on one information source, and $\mathbf{I}_{k}$ is a set of associated Fisher information matrixes of the estimates. In this particular case $N$ is equal to three sources (Gravity Vector, Rate Gyroscopes, and Magnetometers). In order to implement FDI, the statistical agreement between one estimate in the set $\left(e_{s k}, I_{s, k}\right)$ and the remaining estimates that belong to the set $\left(\tilde{\mathbf{E}}_{k}=\mathbf{E}_{k} \backslash e_{s k}, \tilde{\mathbf{I}}_{k}=\mathbf{I}_{k} \backslash I_{s k}\right)$ is defined by evaluating the likelihood that the estimated mean, $e_{s k}$, belongs to the MVN defined by another member of the set, $N\left(e_{i k},\left(I_{i k}\right)^{-1}\right)$

$$
\chi_{s \mid i}^{2}=\left(e_{s k}-e_{i k}\right)^{T} I_{i k}\left(e_{s k}-e_{i k}\right)
$$

where $\chi_{s \mid i}^{2}$ is a scalar that may be used to define a confidence interval that $e_{s k} \sim N\left(e_{i k}\left(I_{i k}\right)^{-1}\right)$.

Recall, that for our case, the inverse of the Fisher information matrix is equation to the errorcovariance matrix. The scalar-value, $\chi_{s \mid i}^{2}$, is used to determine the magnitude of a 'virtual force' 


$$
F_{s \mid i}=\frac{1}{\chi_{s \mid i}^{2}}
$$

which is interpreted as the force of estimate $s$ given sensor $i$. As shown in Equation (10.5) the 'virtual force' magnitude is defined as the inverse of the scalar Chi-Squared test statistic. The direction of each force is them assigned as the vector from $e_{s k}$ to each respective remaining member of $\tilde{\mathbf{E}}_{k}$. The unit vector of which is defined by Equation (10.6).

$$
\vec{u}_{s, i}=\frac{\left(e_{s k}-\tilde{e}_{i k}\right)}{\left\|e_{s k}-\tilde{e}_{i k}\right\|},(i=1: N, i \neq s)
$$

The combined force at $e_{s k}$ is a sum of a total of $N-1$ force vectors associated with $e_{s k}$.

$$
\vec{F}_{s}=\sum_{i=1}^{N} \vec{F}_{s, i},(i \neq s)
$$

Upon summing the 'virtual forces' for each of the sensors that belong to $\mathbf{S}_{k}$, its magnitude, $\left\|\vec{F}_{s}\right\|$, is used as the signal to base the FDI upon. Specifically, if $\left\|\vec{F}_{s}\right\|$ is less than a threshold, $\Omega_{s}$, a failure is declared for the sensors, $s_{k}$. For the general case, this approach is not limited to only three information sources, however in this study only an attitude prediction from rate gyroscopes, and measurement-updates related to Earth's gravity and magnetic vectors. An illustration of the 'virtual force field' associated with the three sources considered in this study is shown in Figure 10.3. 


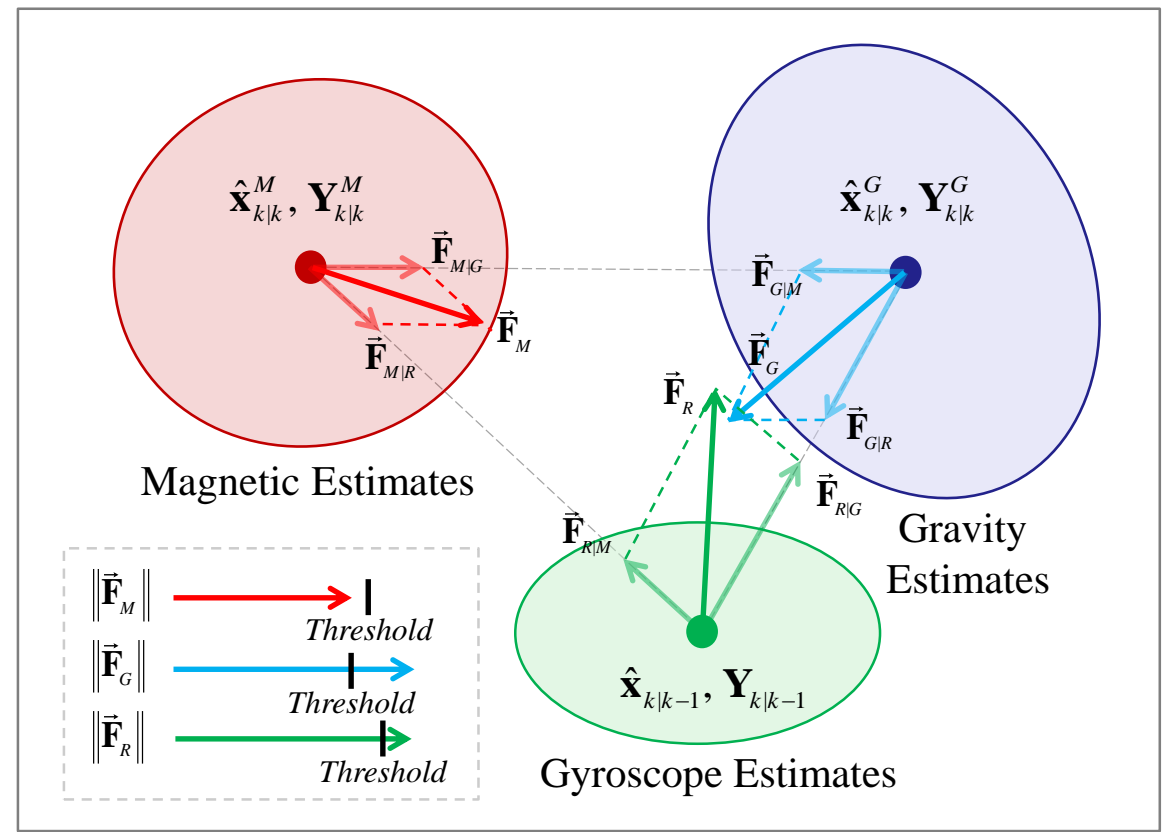

Figure 10.3: 2-D Conceptual schematic of 'Virtual Force Field' FDI [98].

In Figure 10.3 the three ellipses represent the estimated error-covariance of three different information sources. A 'virtual force' magnitude is determined between each estimate and the remaining two. The 'virtual forces' for each of the estimates are summed using vector addition, and their magnitudes are compared to a user-defined threshold. Within this study, the thresholds are determined by monitoring a history of the ' virtual force' magnitudes when the information source is known to be healthy.

\subsection{On-Line Calibration Based Failure Detection and Identification}

While the 'virtual force field' FDI approach of the previous section provides a method for handling relatively large sensor failures, a second approach was implemented to handle more subtle sensor failures. The on-line calibration based method monitors the sensor bias estimates, and assumes that each sensor bias should not exceed a specified bound, $E$. In this case, the 
specified bound was determined by monitoring the magnitude of the time-varying bias during nominal sensor performance. Using this bound, failures are monitored at two levels. The first level triggers if the bias state exceeds, $E$, and at this point, an 'Abnormal Condition' is declared and the on-line calibration is still allowed to accommodate for the sensor bias. However, if the bias then grows to exceed a second threshold, $\alpha E$, where $\alpha>1$, then the set of sensors associated with that sensor bias are removed from the information update procedure. At the point at which a sensor failure is declared, the bias estimate is capped at the detection threshold, and the errorcovariance matrix values associated with the bias states are scaled over time.

$$
P_{k \mid k-1}^{f}=\beta \cdot P_{k \mid k-1}^{f}
$$

For this study, $\beta$ was empirically selected as 1.005 . The purpose of scaling these values over time is to model and increased amount of uncertainty of the bias states that have been capped for some time. Figure 10.4 illustrates this approach.

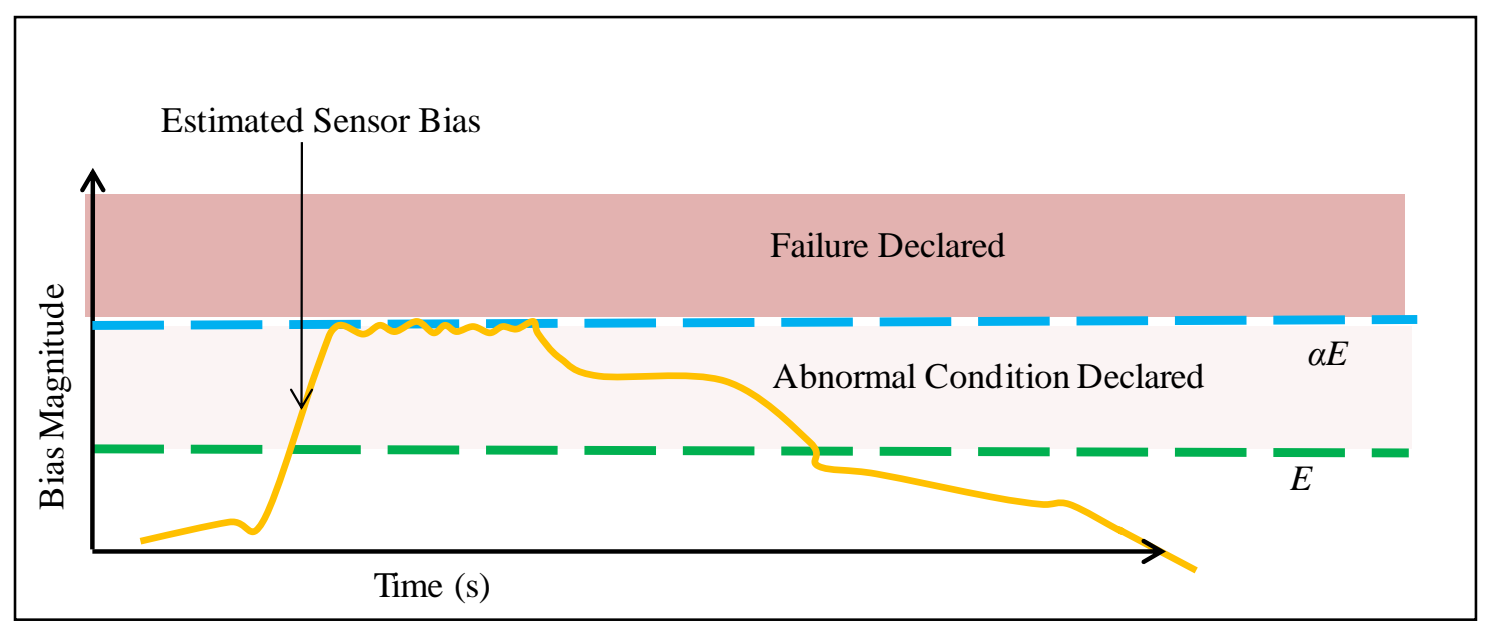

Figure 10.4: Conceptual schematic of on-line calibration based FDI 


\section{5 'Hard' Sensor Failures}

In order to evaluate the fault-tolerant attitude estimation algorithm's ability to handle large abrupt sensor failures ('Hard Failure'), failure scenarios were simulated by imposing specified sensor errors on the actual recorded flight data. In addition, this evaluation was conducted for one half of the flight during the second half of the flight, where:

- A Large GPS velocity measurement failure consisted of abruptly adding random normally distributed noise $N(0,2 \mathrm{~m} / \mathrm{s})$ to the GPS velocity measurements.

- A Large rate gyroscope failure consisted of abruptly saturating all of the rate sensors to their full scale ranges $(150 \mathrm{deg} / \mathrm{sec}$.

- A Large magnetometer consisted of abruptly adding a bias on 500 mGauss on each of the magnetometers.

The results of the sensor fusion algorithm during nominal sensor conditions and during the different failure scenarios are listed in Table 10-1.

Table 10-1: 2-flight average fault-tolerant attitude estimation performance [98].

\begin{tabular}{|c|c|c|c|c|c|c|c|}
\hline GPS/INS/MAG Failure Scenario & $\operatorname{mean}(\mathbf{J})$ & $\operatorname{mean}\left(\left|\boldsymbol{\phi}_{\text {err }}\right|\right)$ & $\boldsymbol{\sigma}\left(\boldsymbol{\phi}_{\text {err }}\right)$ & $\max \left(\left|\boldsymbol{\phi}_{\text {err }}\right|\right)$ & $\operatorname{mean}\left(\left|\boldsymbol{\theta}_{\text {err }}\right|\right)$ & $\boldsymbol{\sigma}\left(\boldsymbol{\theta}_{\text {err }}\right)$ & $\max \left(\left|\boldsymbol{\theta}_{\text {err }}\right|\right)$ \\
\hline Nominal Sensors without FDIA & 1.616 & 1.598 & 1.697 & 6.976 & 1.417 & 1.679 & 6.286 \\
\hline Nominal Sensors with FDIA & 1.584 & 1.585 & 1.708 & 8.549 & 1.360 & 1.610 & 5.527 \\
\hline Large GPS Velocity Failure & 3.773 & 3.566 & 5.546 & 19.545 & 2.205 & 3.185 & 15.503 \\
\hline Large Rate Gyroscopes Failure & 1.897 & 1.8140 & 2.211 & 14.131 & 1.622 & 1.820 & 6.486 \\
\hline Large Magnetometer Failure & 2.061 & 1.931 & 2.216 & 18.134 & 1.693 & 2.240 & 12.016 \\
\hline
\end{tabular}

* All values are reported in degrees

When interpreting Table 10-1, the first good indication is that the performance slightly increases under nominal sensor conditions before and after the FDIA scheme is used (comparing row 1 
and row 2). To account for this, it was determined that the FDIA approach was successful at detecting real instances of poor GPS measurements, and example of this is shown in Figure 10.6.

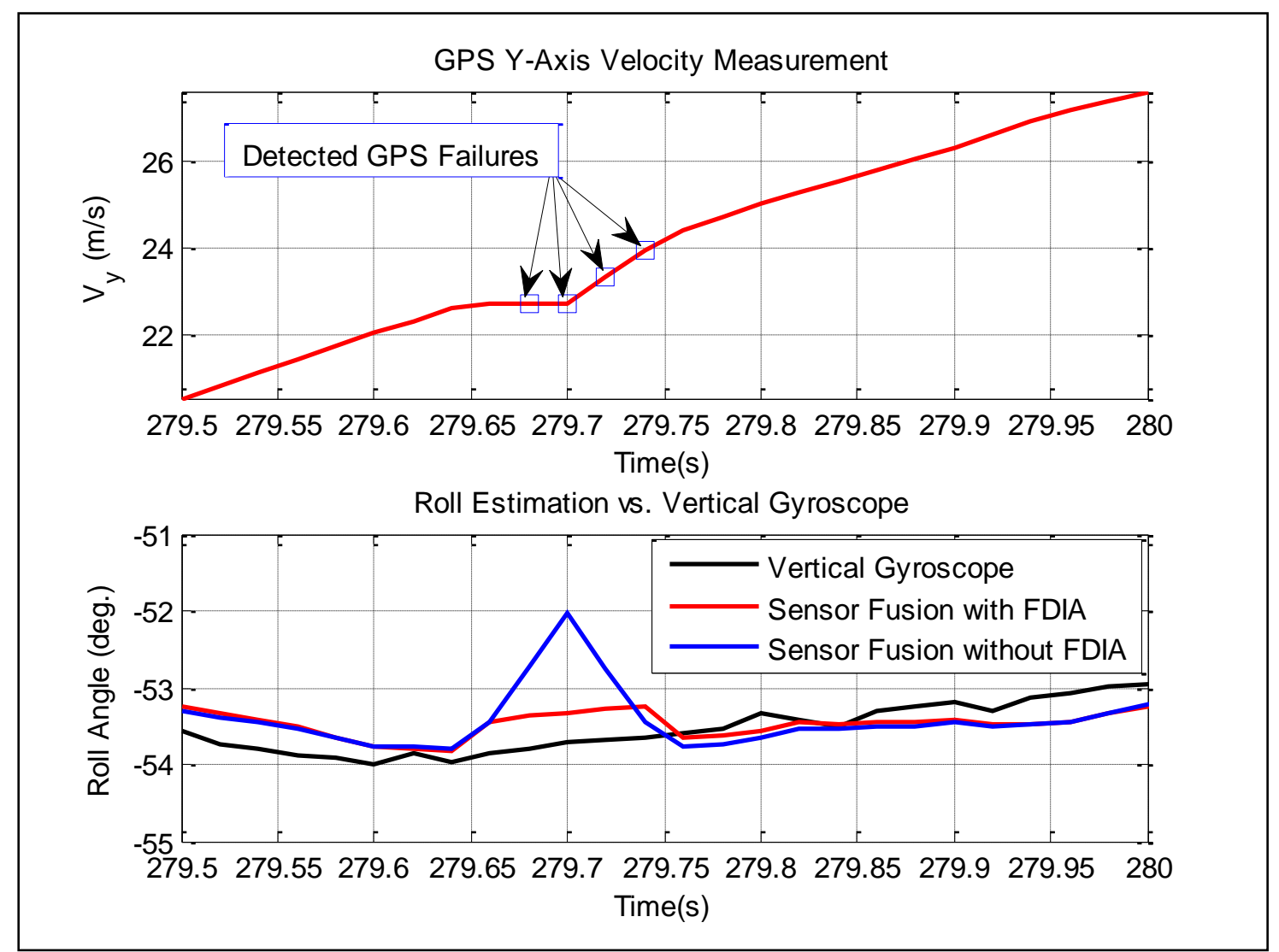

Figure 10.5: 'virtual force field' FDIA of natural GPS measurement quality degradation [98].

In the top plot of Figure 10.6 a half second of flight data is shown, in which a sequence of poor GPS measurements are detected. The poor GPS measurement effect on the roll estimates is shown in the bottom plot of Figure 10.6 in which the sensor fusion with FDIA tracks the vertical gyroscope roll channel more closely. The bottom three rows of Table 10-1 indicate the estimation performance of the sensor fusion algorithm during the failure scenarios. In general, the GPS failure was the worst-case scenario, but still provides a reasonable solution with an average estimation performance under $4^{\circ}$. This is expected, due to the fact the INS/Magnetometer sensor fusion formulation presented in Chapter 9 resulted in the worst 
attitude estimates among all the sensor fusion algorithms. The estimation performances during either the rate gyroscope or the magnetometer failure scenarios were very good, in that both were within $0.5^{\circ}$ of the nominal case.

In order to illustrate the FDI scheme, Figure 10.5 shows the 3-D error-covariance ellipsoids located at the estimated attitude mean, as well as the 'virtual force' vectors of the three sensors. In Figure 10.6, the green ellipsoid and vector indicate the rate gyroscope prediction, the blue ellipsoid and vector indicate the GPS/Accelerometer information fused with the random walk prediction, and the red ellipsoid and vector indicate the magnetometer information fused with the random walk prediction.

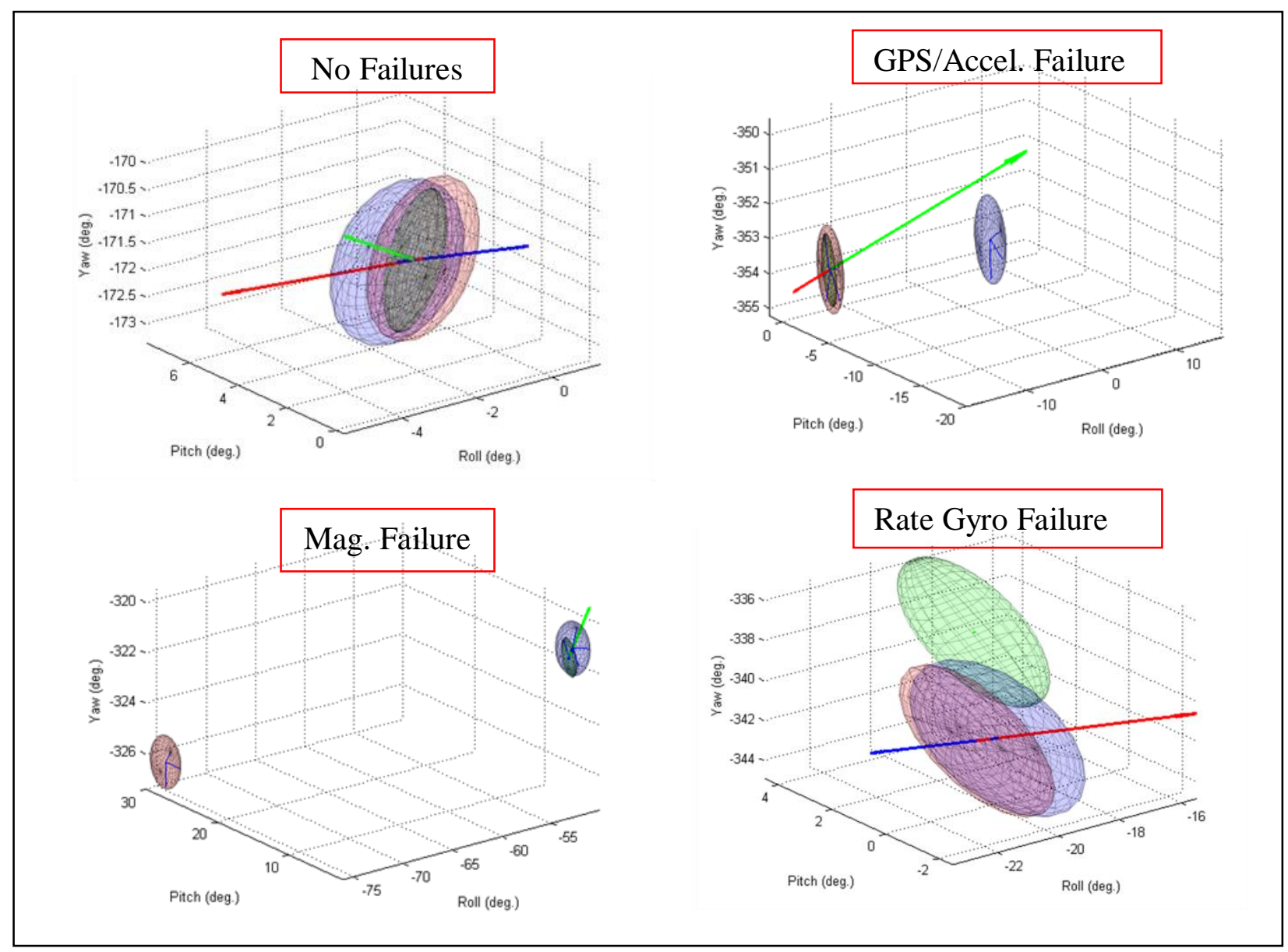

Figure 10.6: Snapshots of 'virtual force field' detection during sensor failures. 
In the top left of Figure 10.6, there is no sensor failure. In this case all three error-covariance ellipsoids are intersecting one another, and the three 'force' vectors are all pointing toward a common mean. In the top right of Figure 10.6, a large GPS failure was introduced. In this scenario, the blue error-covariance ellipsoid is far away from the other two error-covariance ellipsoids and the blue force vector is not even visible because it is such a small magnitude. In addition the two remaining healthy sensor's 'force vectors' are pointing toward one another. The same trend is followed during the two other failure scenarios as indicated in the bottom half of Figure 10.6.

In order to further demonstrate the 'virtual force field' approach, a simulation was conducted in which 3 sequential 15 second duration sensor failures were imposed on the flight data, with 15 seconds of nominal sensor performance between each failure. This test was intended to show the smooth transitions between accommodating for different types of sensor failures. The roll estimation and roll estimation error during this simulation is shown in Figure 10.7 and the pitch estimation and estimation error are shown in Figure 10.8. 




Figure 10.7: 'virtual force-field' FDIA roll estimation performance during 315 second failures [98]. 

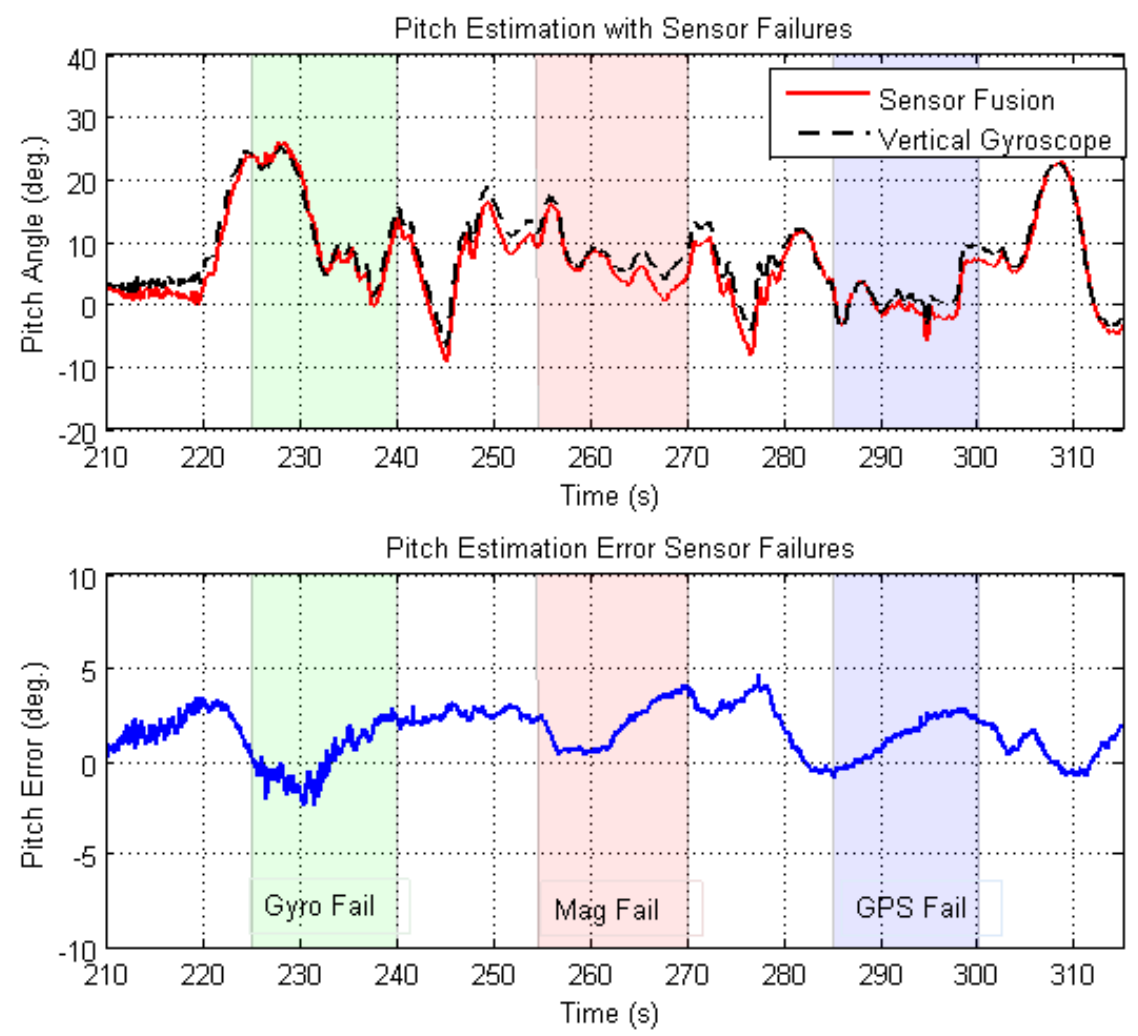

Figure 10.8: 'virtual force-field' FDIA pitch estimation performance during 315 second failures.

Figures 10.7 and 10.8 show the smooth transitions between failure anre nominal scenarios. In addition, as expected the estimation error does tend to get slightly more noise during failed condition. Figure 10.9 shows the logorithmic scale of the force magnitudes of the three sources of information throught the 3 sequential 15 second failure simulation. 


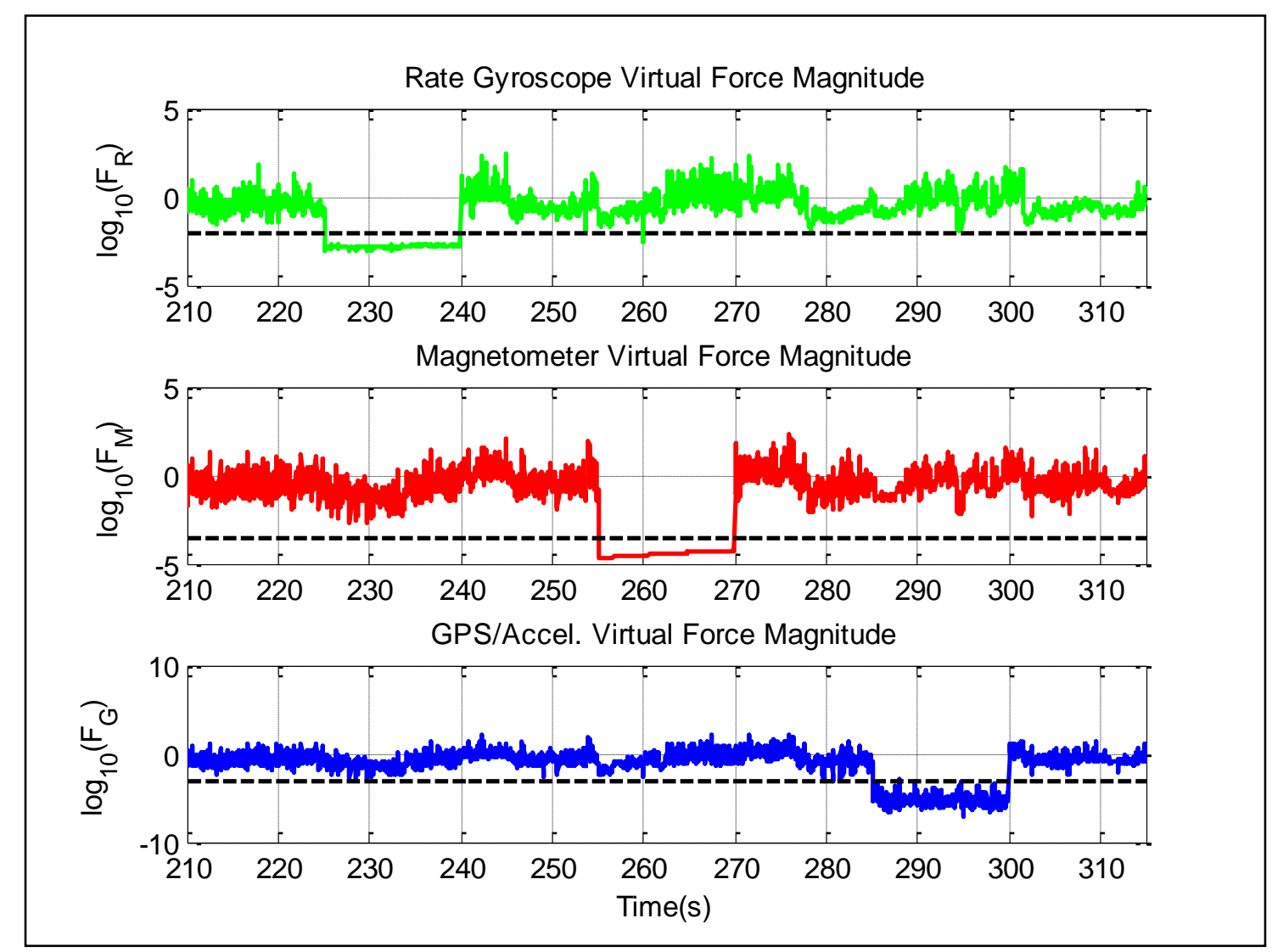

Figure 10.9: Magnitudes of 'virtual forces' and associated failure detection threshold for each of the three information sources [98].

As Figure 10.9 demonstrates, the 'virtual force field' FDI scheme provides a clean signal to use for detecting large abrupt sensor failures. In Figure 10.9, the black dashed lines indicate the user-defined detection thresholds, $\Omega$.

While one might argue that it would be relatively easy to determine large sensor failures after a few time-steps, it is important to stress that the 'virtual force field' detection scheme instantaneously, automatically, handles detecting and accommodating for large sensor failures. In addition, large sensor failures are accommodated with smooth as indicated in Figures 10.7 and 10.8. However, in order to further test the 'virtual force field' FDI strategy's ability to handle 
sensor failures, that are large and abrupt, but instead only short duration, a simulation was conducted in which measurement spikes were introduced. As an example, this test was performance by biasing the rate gyroscopes; however, it is reasonable to assume the same trend would hold for the other two information sources. For this test, randomly generated biases that are distributed $N(0,100 \mathrm{deg} / \mathrm{s})$ were introduced on all three rate gyroscopes with a uniform probability of occurrence of $5 \%$. Figure 10.10 shows the outcome of this test.

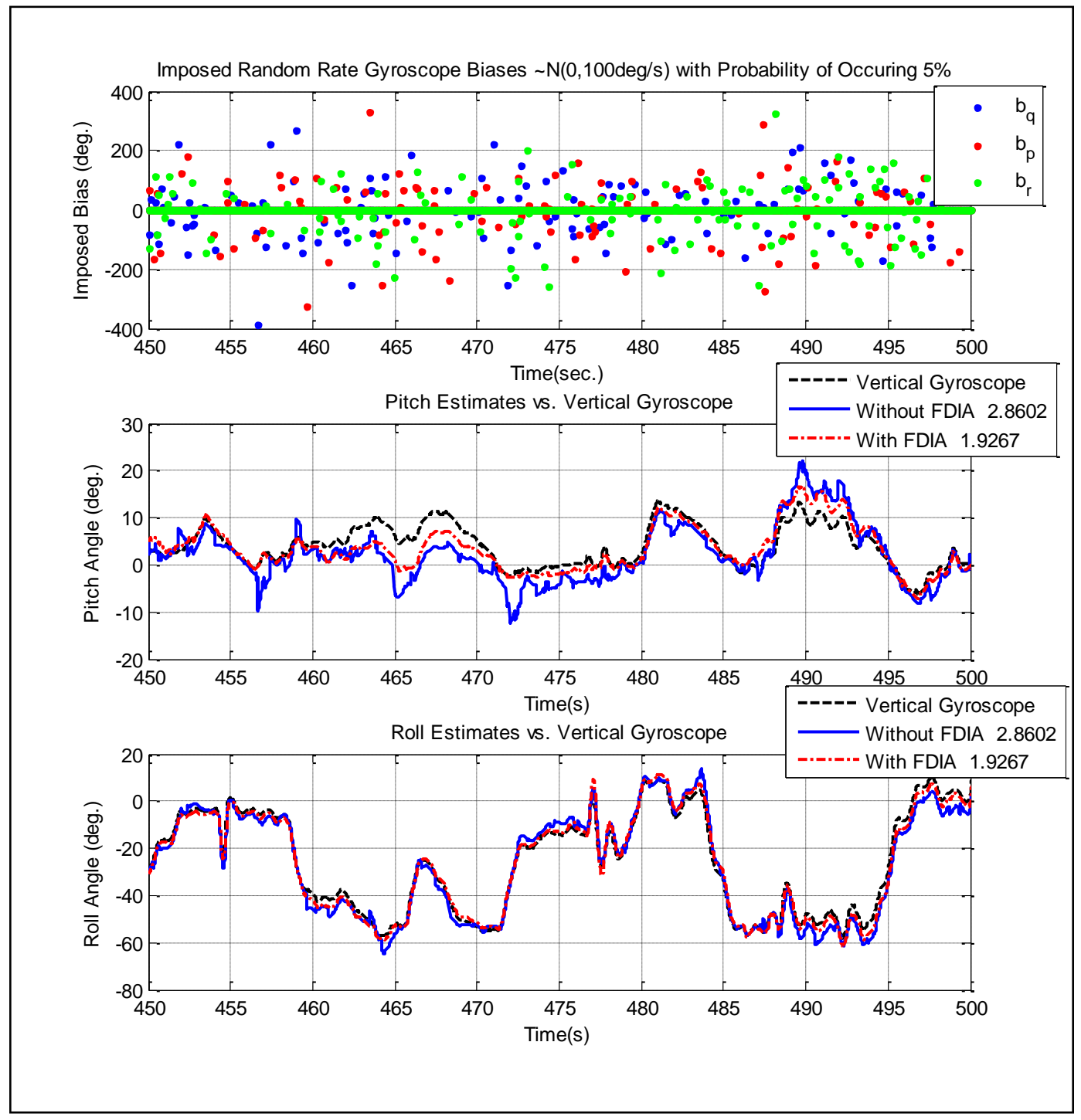

Figure 10.10: Randomly generated rate bias 'virtual force-field' FDIA test. 
Figure 10.10 indicates the sensor fusion with FDIA shows a better performance to large, but short duration sensor failures. However, when viewing the average estimation error, it is important to mention the sensor fusion algorithm without FDIA is fairly robust to sensor failures. Particularly on the pitch channel, which is plotted in the center of Figure 10.10, the sensor fusion scheme with FDIA is much smoother.

\section{6 'Soft' Sensor Failures}

In addition to handling large sensor failures, it is important to handle small failures that slowly grow over time. To accommodate for these types of failures, the on-line calibration based FDI scheme was used. Throughout this section, some case study examples of the on-line calibration based FDI approach are discussed.

In order to show an example of this type of failure accommodation, a simulation was conducted in which a known time-varying bias was imposed on the flight data. In particular, Figure 10.11 shows an example in which a small pitch rate bias was introduced that was driven by random walk with a standard deviation $0.02 \mathrm{deg} / \mathrm{s}$. 


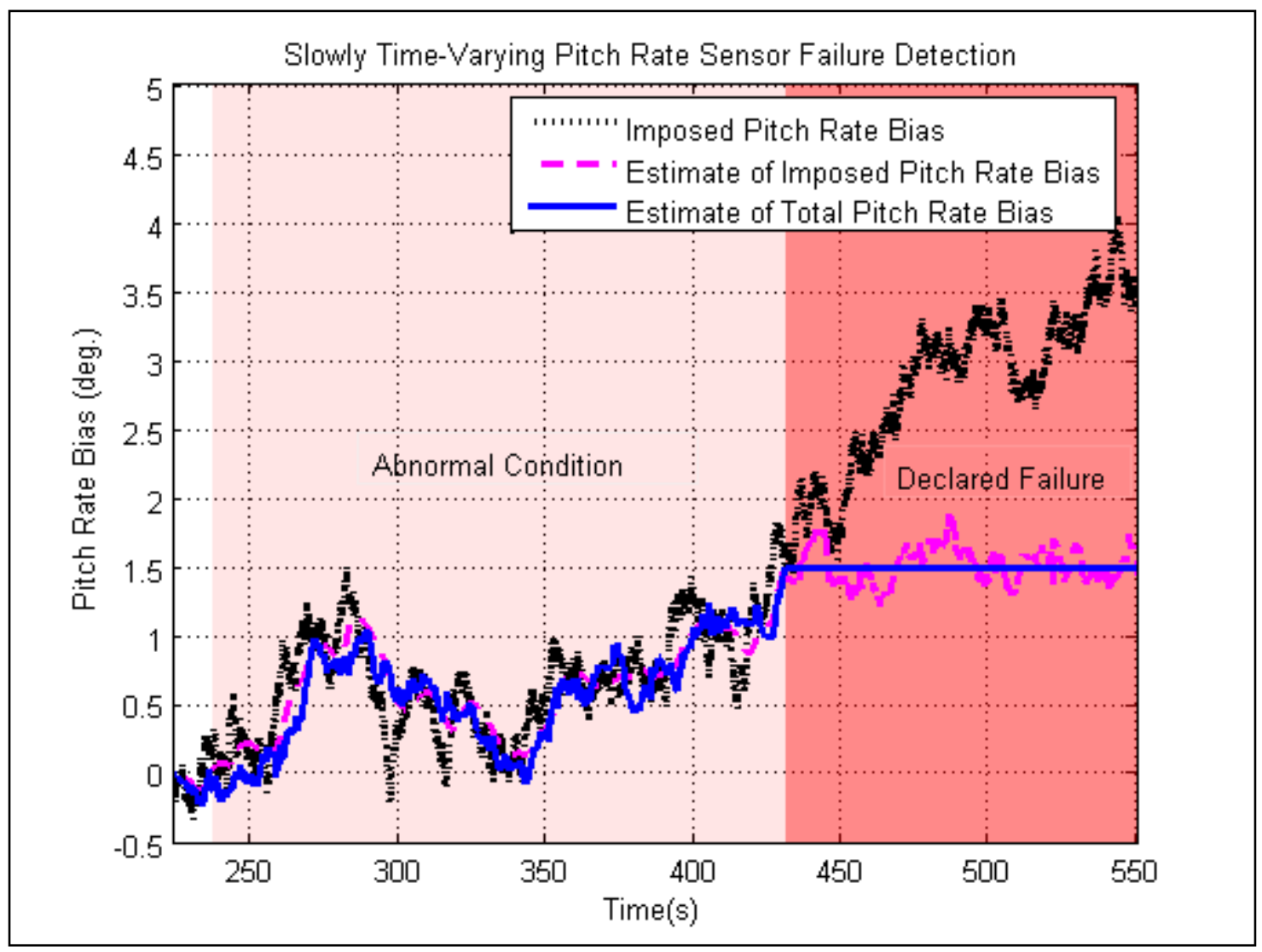

Figure 10.11: FDIA of small pitch rate bias through on-line calibration tracking [98].

As indicated in Figure 10.11, for a significant portion of the flight, an abnormal condition is declared, but the on-line calibration estimate is allowed to accommodate for the small bias. The failure declaration threshold was set to be $1.5 \mathrm{deg} / \mathrm{s}$, at around $440 \mathrm{~seconds}$, the bias estimate is capped, as indicated by the blue signal, and the rate gyros are no longer used. It is important to point out that this simulation imposed an artificial time-varying bias on a real flight data signal that already had a natural time-varying bias; therefore in order to obtain the estimate of the imposed bias (magenta dashed signal), baseline bias estimated were subtracted from the signal after the fact for plotting purposes.

Another simulation was conducted with a similar scenario, however with a bias that grows and then shrinks. The purpose of this test was to test the ability of the FDI strategy to 
'turn-off'. Figure 10.12 show the simulation in which s pitch rate bias was introduced, grew linearly, and then got smaller linearly.

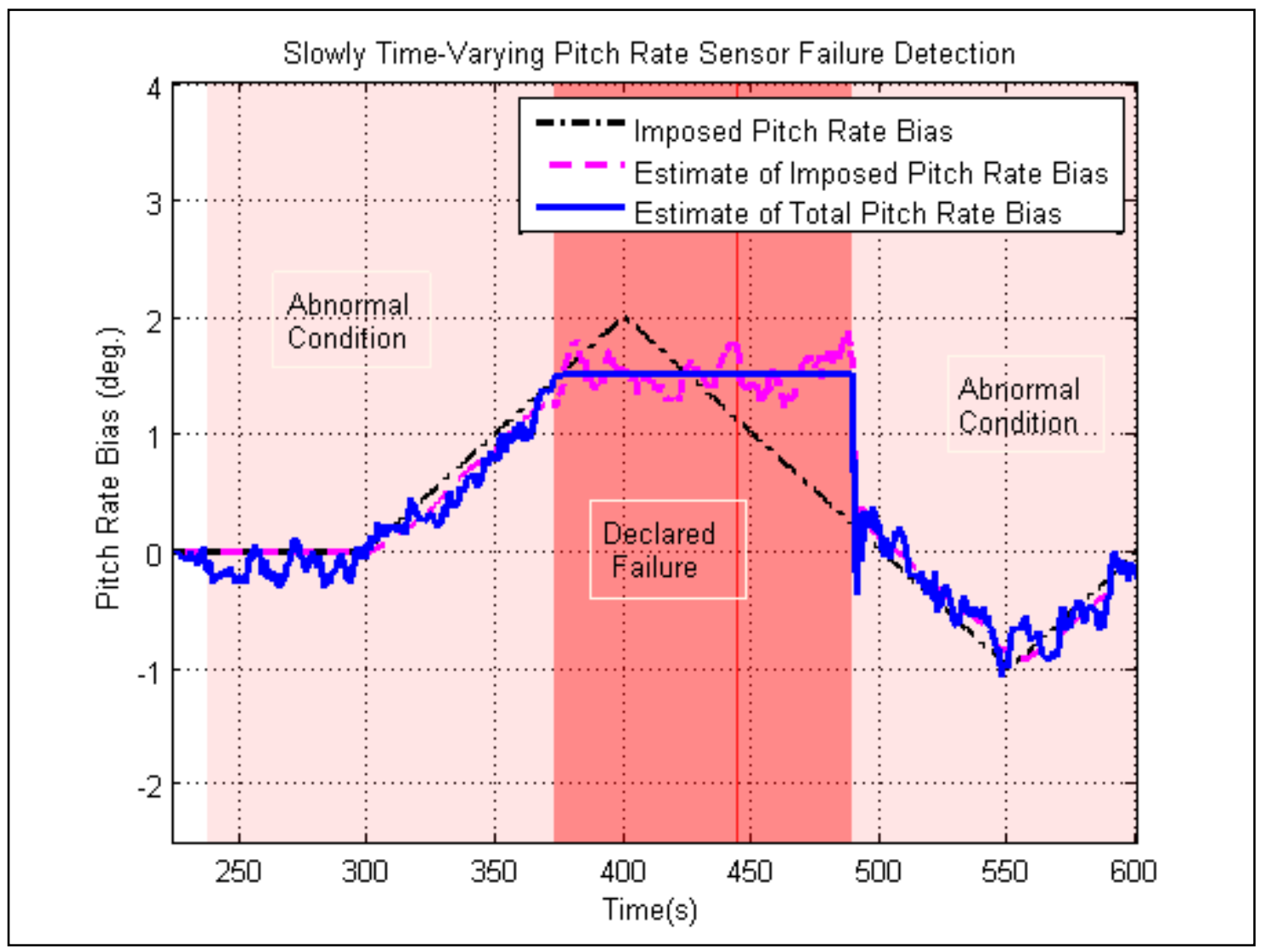

Figure 10.12: FDIA of small pitch rate bias through on-line calibration tracking, 'turn-off' test.

Figure 10.12 shows that on the way up, there is hardly any time lag between the bias estimate, and the artificially imposed bias; however, it took the accommodation strategy some time to allow the sensors to be used again. This is due to the fact that, with this approach, upon a failure declaration, the bias estimate is capped at the detection threshold. Although it is revaluated at each time step, the imposed bias must be significantly smaller for the estimated bias to jump below the threshold. Therefore, this approach is conservative, in that, it is more difficult for a sensor to be re-declared healthy, than for it to be declared faulty. 
So far, cases study of a small pitch rate bias detection have been shown, but these result are also shown with respect to magnetometer failures. Figure 10.13 indicates the detection of a slowly growing magnetometer bias on the $\mathrm{x}$-axis magnetometer, which was driven by random walk with standard deviation $2 \mathrm{mGauss}$ (note that the range of the sensors is \pm 3500 mGauss).

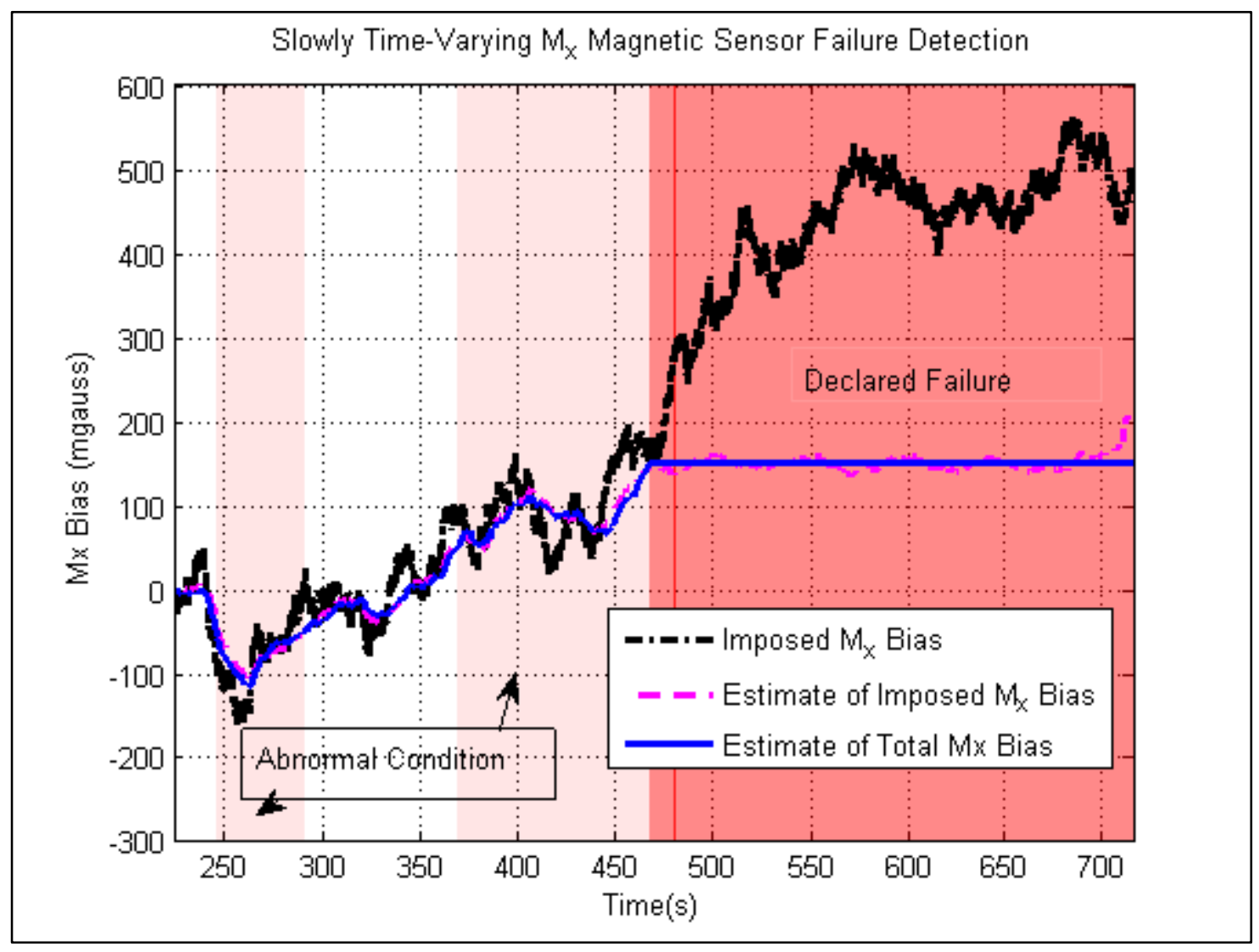

Figure 10.13: FDIA of small magnetometer bias through on-line calibration tracking.

The case shown in Figure 10.13 shows the ability for the detection scheme to go in and out of declaring an abnormal condition once the bias growth changes directions. In this instance, the calibration failure detection threshold was set to 150 mGauss. Figure 10.14 shows the effect of FDIA for the above scenario. 


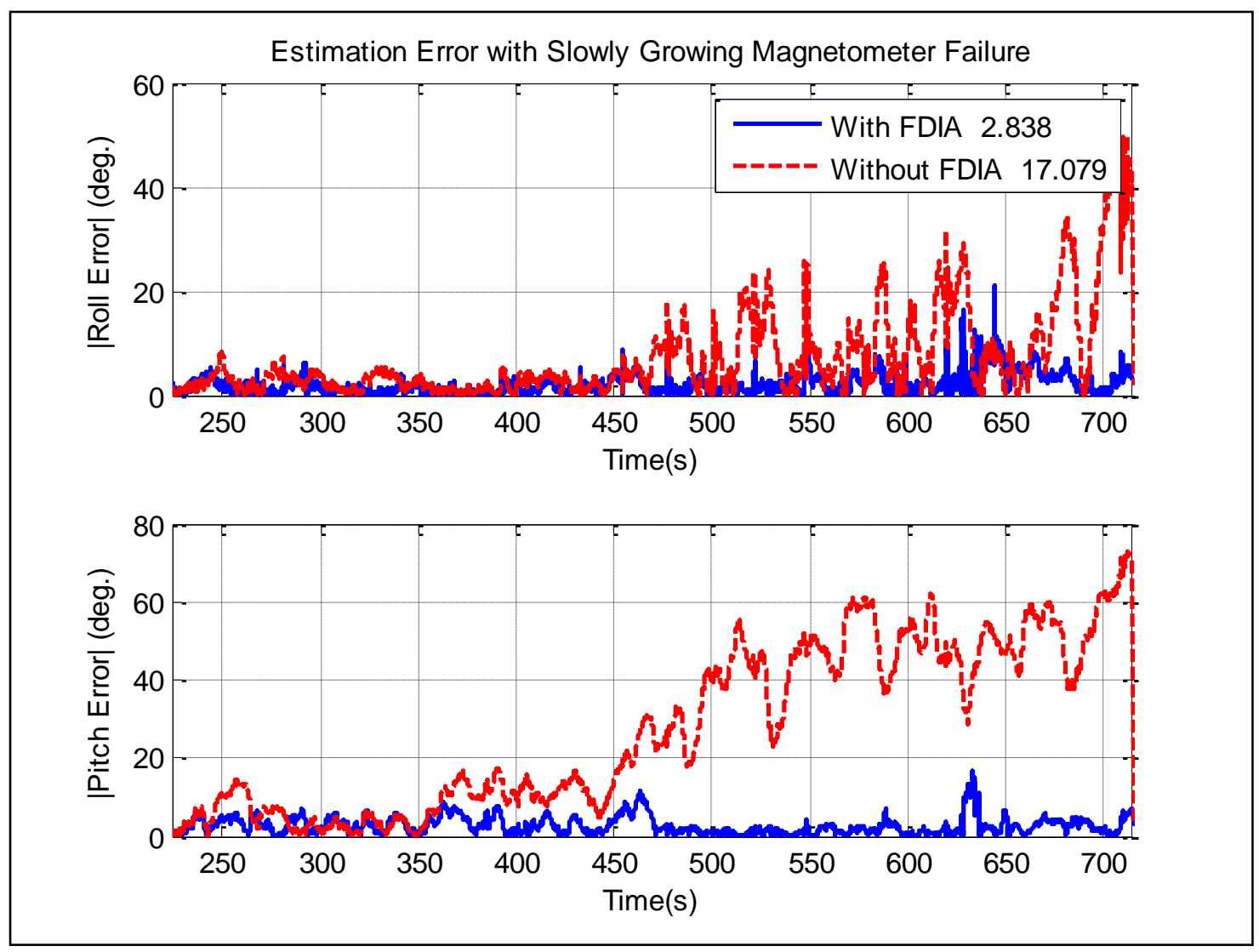

Figure 10.14: Sensor Fusion estimation error with and without FDIA during a growing magnetometer bias.

Figure 10.14 further indicates the importance of FDIA. With the growing magnetometer bias, the sensor fusion estimates without FDIA continue to grow, while the estimation error of sensor fusion with FDIA remains small.

Finally, the same trend is exhibited during a simulation in which a slowly growing accelerometer error is imposed on the accelerometer signal. In the simulation shown in Figure 10.15 the imposed accelerometer bias was random walk driven with a standard deviation of 0.2 $\mathrm{m} / \mathrm{s}^{2}$ 


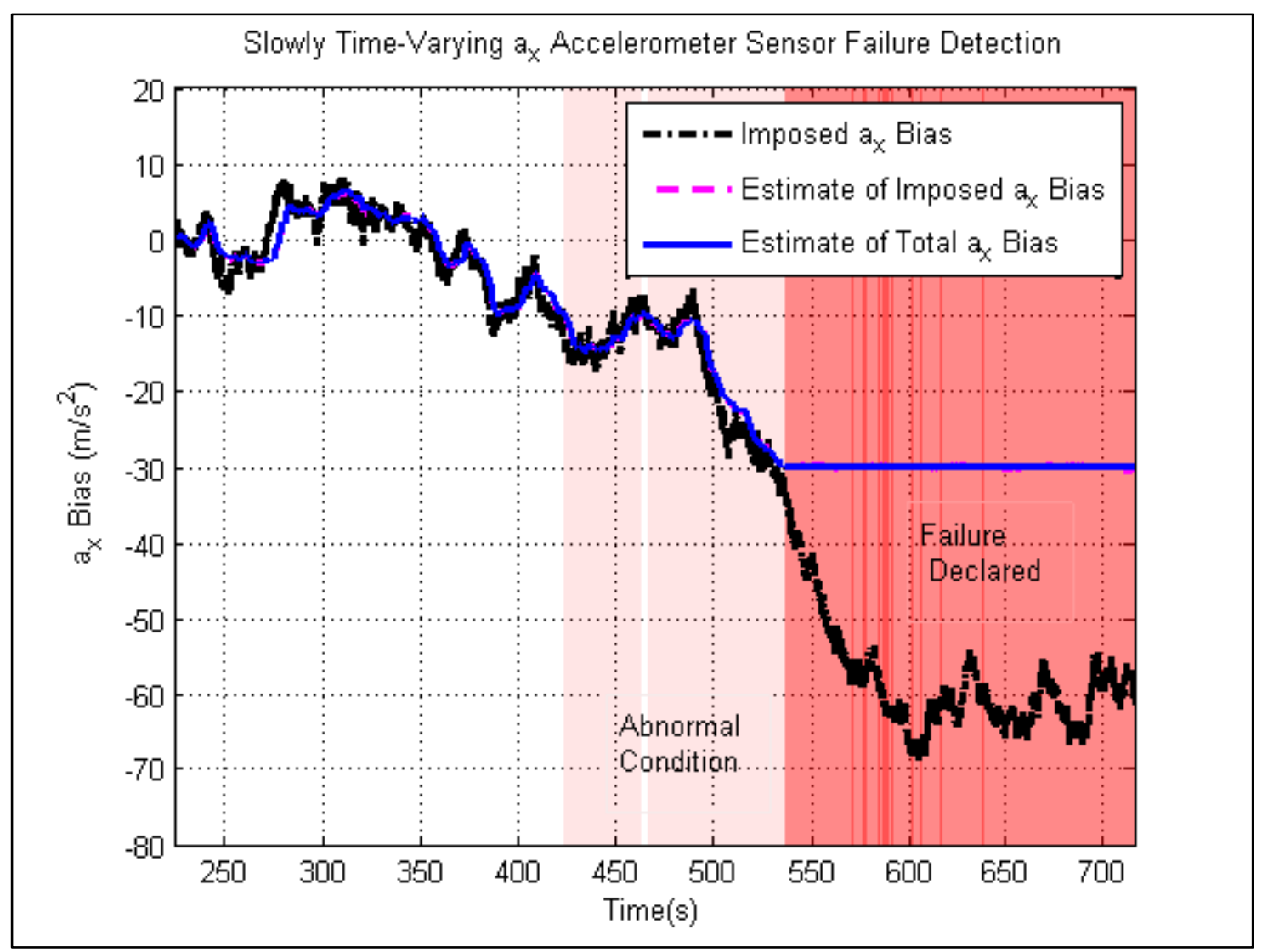

Figure 10.15: FDIA of growing accelerometer bias through on-line calibration tracking.

Just as the case of the magnetometers and rate gyroscopes, monitoring the sensor biases estimated on-line proves successful for detecting slowly growing failures. The estimation performance during this simulation is shown in Figure 10.16 


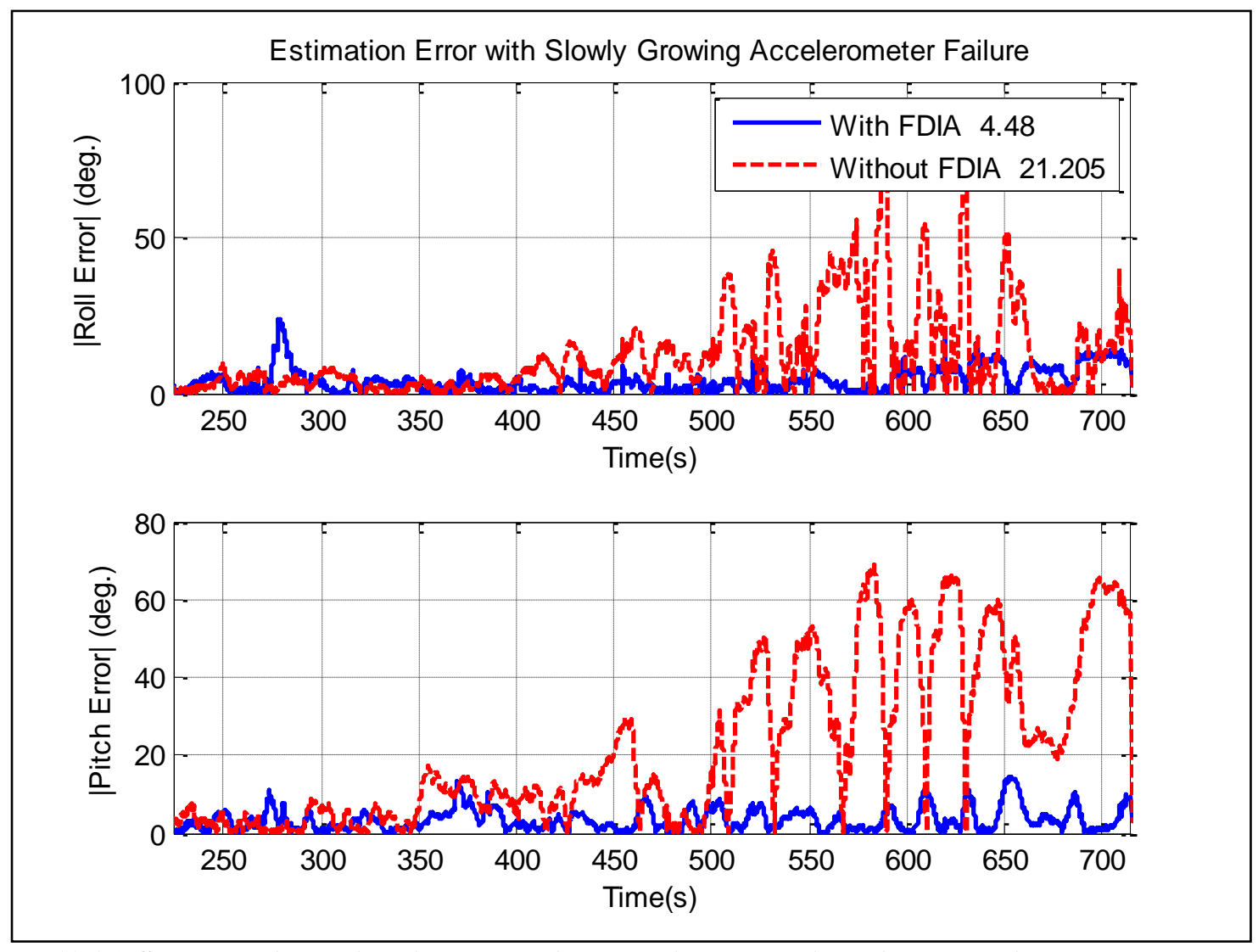

Figure 10.16: Sensor Fusion estimation error with and without FDIA during a growing accelerometer bias.

As Figure 10.16 indicates, the estimation error with FDIA is significantly smaller that without. Once the bias threshold of the accelerometer is reached, the entire GPS/Accelerometer based measurement-update is removed from the information filter update. Therefore, the estimation error even with FDIA is fairly large. This is comparable with the large GPS failure scenario listed in Table 10-1, and is expected due to the poor performance of the 3-state INS/Magnetometer formulation discussed in Chapter 9. 


\section{Chapter 11 : Conclusions and Future Directions}

\subsection{Summary and Conclusion}

In SUAV based applications the performance of navigation systems often reflects a tradeoff between accuracy and cost. In order to compensate for this, sensor fusion algorithms based on nonlinear Kalman Filters are often used to provide a low-cost and accurate solution. Within this study, several sensor fusion algorithms were formulated and experimentally validated in terms of estimation performance. This provided a necessary supplement to previously conducted simulation studies in the available literature $[31 ; 41 ; 40]$, and provided a foundation of which to build upon toward designing a fault-tolerant algorithms.

Following a detailed comparative analysis, it was determined through the use of experimental flight data the EKF's and SPKF's ability to handle the nonlinearities and uncertainties associated with the application of attitude estimation for these low-order estimation filters are essentially the same. The trend of very similar estimation performance was shown across 23 diverse sets of GPS/INS flight data collected using four separate sensor systems with the use of independent attitude 'truth' measurements. Furthermore, within this study, multiple formulations were considered which varied how noise was considered and where nonlinear relationships were used in the estimation filter. In addition, a linearization comparison was conducted in which the central difference between the EKF and SPKF was directly compared. This analysis also showed little difference between the two approaches. Specifically, both the time-varying linearized models extracted using the statistical linearization approach of the UKF and the analytical linearization used by the EKF were very similar. Across many flights with different 
hardware and among different algorithm formulations, EKF and UKF provided similar SUAV attitude estimation performance.

The experimental EKF and UKF comparison results are valuable for others considering the particular application of SUAV attitude estimation. On the other hand, this work does not question the fact that the Unscented Transformation is fundamentally a better way to handle the transformation of random variables through nonlinear functions [30]. In fact, it is likely that applications with other nonlinearities may see performance improvements with the sigma-point approach; however it just was not the case for this particular application. The comparisons offered in this study were exposed to nonlinearities such that the UKF on the average did not outperform the EKF.

Another aspect of this study used the nonlinear KFs to improve accuracy by tracking on-line sensor biases. This approach is common in the literature [23; 32; 24], but within in this study it represented an important milestone toward achieve fault-tolerance through sensor fusion. In particular, when provided a simple model of time varying sensor biases, both the EKF and SPKF were experimentally shown to track time-varying inertial sensor biases and improve attitude estimation performance. Another portion of this study used a GPS/INS sensor fusion solution to determine a calibration model of a set of low-cost tri-axial magnetometers. Using the GPS/INS derived magnetometer calibration model, improved GPS/INS/Magnetometer attitude estimation sensor fusion performance, and provided an additional partially redundant source of information. On-line calibration within the filters improved performance and opened a path toward faulttolerant attitude estimation.

To further address reliability, a sensor fusion attitude estimation algorithm was developed that provided a high-performance solution both during nominal sensor conditions and during 
simulated sensor failures. This algorithm was based on a nonlinear Information Filter (i.e. UIF). The UIF provided a natural platform for failure accommodation, as was suggested in the literature for other sensor fusion applications $[22 ; 20 ; 21]$. In order to implement FDI, a twotiered approach was used. This FDI approach was based on statistical hypothesis testing and online bias monitoring. In particular statistical hypothesis testing was used to populate a 'virtual force-field' which provided a scalable way to evaluate the health of individual information sources. The combination of these approaches provided a solution for mitigating both abrupt sensor failures and failures that grew from zero over time. By imposing artificial failures to corrupt real flight data, several simulation case studies were shown in which the fault-tolerant attitude estimation algorithm successfully identified and accommodated for a sensor failure.

Finally, a contribution of this work is the development of an SUAV avionics system for future research at WVU. The system is specifically designed to allow for fault-tolerant flight controls research, and provides a lot of flexibility for future sensor fusion research.

\subsection{Future Directions}

There are many openings for future development in this project. In particular, while the random walk assumption for on-line bias estimation proved powerful, an in-depth stochastic modeling of the inertial sensors based on Allan variance testing $[91 ; 102 ; 50 ; 92 ; 103]$ could provide a better estimation of the true time-varying sensor bias. This is the next step planned by the author of this dissertation.

Furthermore, while this study leads to the conclusion that there is essentially no difference between EKF and UKF for this practical application, a sensitivity analysis to the various design parameters associated with EKF and UKF as well as their robustness to various conditions would 
be useful for further investigation. This is the graduate project of fellow WVU PhD student Matthew Rhudy [97].

Finally, in this study only three information sources were used for the fault-tolerant attitude estimation algorithm. Because at high-level the failure accommodation strategy outlined in this study is a form of voting, by increasing the number of information sources, it is expected that better performance, robustness, and the ability for the algorithm to accommodate for concurrent failures. This is a direction that is currently under development for experimental evaluation by Dr. Yu Gu. 


\section{References}

[1] Hall, D. L. and Linas, J., "An Introduction to Multisensor Data Fusion." Proceedings of IEEE, January 1997, Issue 1, Vol. 85, pp. 6-23. Invited Paper.

[2] , Home Page: ISIF. International Society of Information Fusion. [Online] [Cited: September 10, 2010.] www.isif.org.

[3] Lewantowicz, Z., "Arhitectures anf GPS/INS Integration: Impact on Mission Accomplishment." IEEE Aerospace and Electronic Systems Magazine. June, 1992.

[4] El-Sheimy, N., Hou, H. and Niu, X., "Analysis and Modeling of Inertial Sensors Using Allan Variance." IEEE Transactions on Instrumentation and Measurement, s.l. : IEEE, January 2008, Issue 1, Vol. 57. 0018-9456.

[5] Syed, Z. F., Aggarwal, P., Goodall, C., Niu, X., El-Sheimy, N., "A New Multi-Position Calibration Method for MEMS Inertial Navigation Systems." Measurement Science and Technology, s.1. : IOP Publishing, May 2007, Issue 18, pp. 1897-1907. 0957-0233.

[6] Grewal, M. S., Weill, L. R and Andrew, A.P., Global Positioning, Inetial Navigation \& Integration. 2nd. New York, NY : John Wiley \& Sons, 2007.

[7] Guo, P., et al., "The Soft Iron and Hard Iron Calibration Method using Extended Filter for Attitude and Heading Reference System." Monterey, CA : s.n., May, 2008. Position, Location and Navigation Symposium. pp. 1167 - 1174 . 978-1-4244-1536-6.

[8] Rogers, Robert M., Applied Mathematics in Integrated Navigation Systens. [ed.] Joseph A. Schetz. 2nd Edition. Reston : American Institute of Aeronautics and Astronautics, Inc., 2003.

[9] Titterton, D. and Weston, J., Strapdown Inertial Technology. 2nd Edition. Herts, UK: The Institution of Electional Engineers/ AIAA, 2004. ISBN 0863413587.

[10] O'Donnell, C. F., [ed.]., Inertial Navigation Analysis and Design. New York : McGraw Hill, 1964.

[11] Pitman, G. R., [ed.]., Inertial Guidance. New York : John Wiley and Sons, 1962.

[12] Kaplan, E. and Heagarty, C., Understanding GPS Principles and Applications. 2nd. Norwood, MA : Arttech House, 2006. 
[13] Bowring, B., "Transformation from Spatial to Geographical Coordinates." Survey Review, s.l. : Maney, Issue 181, Vol. 23, pp. 323-327.

[14] , The GPS System: Sources of Error. kowoma.de. [Online] 2009. [Cited: April 3, 2011.] http://www.kowoma.de/en/gps/errors.htm.

[15] Chen, Z., Bayesian Filtering: From Kalman Filters, to Particle FIlters, and Beyond. Technical Report, McMaster University. [Online] 2003. [Cited: December 20, 2009.] www.math.ubordeaux.fr/ delmoral/chen_bayesian.pdf.

[16] Arulampalam, S. M., Gordon, N. and Clapp, T., "A Tutorial on Particles Filters for Online Nonlinear/Non-Gaussian Bayesian Tracking." IEEE Transactions on Signal Processing, 2002, Issue 2, Vol. 50.

[17] Doucet, A., de Freitas, N and Goron, N., [ed.]., Sequential Monte Carlo Methods in Practice. 2001.

[18] Kalman, R. E. and Bucy, R. S., "New Results in Linear Filtering and Prediction Theory." Journal of Basic Engineering (Transactions of ASME), pp. 95-108.

[19] Julier, S. and Uhlmann, J., "A New Extension of the Kalman Filtering to Non Linear Systems." SPIE Proceesinfs Series, s.l. : IEEE, 1997, Vol. 3069, pp. 50-62.

[20] Mutambara, A. G. O., Decentralized Estimation and Control for Multisensor Systems. Washington D.C. : CRC Press, 1998. 0-8493-1865-3.

[21] Vercauteren, T. and Wang, X., "Decentralized Sigma-Point Information Filters for Target Tracking in Collaborative Sensor Networks." IEEE Transactions on Signal Processing, 2005, Issue 8, Vol. 53.

[22] Lee, D.-J., "Unscented Information Filtering for Distributed Estimation and Multiple Sensor Fusion." Honolulu : American Institute of Aeronautics and Astronautics, 2008. AIAA Guidance, Navigation and Control Conference and Exhibit. AIAA 2008-7426.

[23] Simon, D., Optimal State Estimation. Hoboken : John Wiley \& Sons, 2006. 13 978-0-471-70858-2.

[24] Crassidis, John L. and Junkins, John L., Optimal Estimation of Dynamic Systems. New York: Chapman \& Hall/ CRC, 2004.

[25] Stengel, Robert F., Optimal Control and Estimation. New York : Dover Publications, Inc., 1994.

[26] Bin, W., Jian, W. and Jianping, W.1 Baigen, C., "Study on Adaptive GPS/INS Integrated Navigation Systems." IEEE Transactions on Intelligent Transport Systems, Oct. 2003, Vol. 2, pp. 1016-1021. 
[27] Saisadek, J., Wang, Q. and Zeremba, M., "Fuzzy Adaptive Kalman Filtering for INS/GPS Data Fusion." Rio, Patras, Greece : s.n., July, 2000. 15th IEEE International Symposium in Intelligent Control.

[28] Chiang, K-W and Huang, Y-W., "An Intelligent Navigator for Seamless INS/GPS Integrated Land Vehicle Applications." Applied Soft Computing, Jan. 2008, Issue 1, Vol. 8, pp. 722-733.

[29] Jwo, D-J and Huang, H-C., "GPS Navigation Using Fuzzy Neural Network Aided Adaptive Extended Kalman Filter for DGPS Positioning." Sevill, Spain : s.n., December, 2005. 44th IEEE Conference on Decision and Control, and the European Control Conference.

[30] Julier, S. and Uhlmann, J., "Unscented Filtering and Nonlinear Estimation." Proceddings of IEEE, March 2004, Issue 3., Vol. 92.

[31] van der Merwe, R., Wan, E. and Julier, S., "Sigma-Point Kalman Filters for Nonlinear Estimation and Sensor Fusion- Applications to Integrated Navigation." Providence, RI : AIAA, 2004. AIAA Guidance, Navigation and Control Conference. 2004-5120.

[32] van der Merwe, R., Sigma-Point Kalman Filters for Probabilistic Inference in Dynamic State-Space Models. Electrical and Computer Engineering, Oregon Health and Science University. 2004. $\mathrm{PhD}$ Thesis.

[33] Wan, E. and van der Merwe, R., "The Unscented Kalman Filter for Nonlinear Estimation." Lake Loise, Alberta, CA : IEEE, 2000. Proceedings of the IEEE Sysposium (AS-SPCC).

[34] Lefebvre, T., Bruyninckx, H., De Schuller, J., "Comment on "A new method for the nonlinear transformation of means and covariances in filters and estimators"." s.l. : omatic Control, IEEE Transactions on, 2002, Issue 8, Vol. 47, pp. 1406 - 1409.

[35] St. Pierre, M. and D., Ing., "Comparison Between the Unscented Kalman Filter and the Extended Kalman Filter for the Position Estimation Module of an Integrated Navigation Information System." Parma, Italy : s.n., 2004. IEEE Intelligent Vehicles Symposium.

[36] Ford, T., et al., "OEM4 Inertial: A Tightly Integrated Decentralized Inertial/GPS Navigation System." Salt Lake City, UT : s.n., Sep. 2001. Proceedings of ION GPS '01.

[37] Maybeck, P., Stochastic Models, Estimation, and Control. New York : Academic Press, 1979. Vol. I.

[38] Pinson, J.C., "Inertial Guidance for Cruise Vehicles." [ed.] C. T. Leondes. Guidance and Control of Aerospace Vehicles, New York : McGraw-Hill, 1963. 
[39] Evans, Curtis D., The Design and Analysis of Integated Navigation Systems Using Real INS and GPS Data. Wright-Patterson Air Force Base, OH : Air Force Institute of Technology, 1994. Masters Thesis.

[40] Crassidis, J., "Sigma-Point Filtering for Integrated GPS and Inertial Navigation." San Francisco, CA : s.n., 2005. AIAA Guidance, Navigation and Control Conference and Exhibit.

[41] Wendel, J., et al., "A Performance Comparison of Tightly Coupled GPS/INS Navigation Systems Based on Extented and Sign Point Kalman Filters." Navigation: Journal of the Tinstitute of Navigation, Spring 2006, Issue 1, Vol. 53.

[42] Stastny, N., Bettinger, R. and Chavez, F., "Comparison of the Extended and Unscented Kalman Filters for Angles Based Relative Navigation." Honolulu, HI : s.n., Aug. 2008. AIAA/AAS Astrodynamics Specialist Conference.

[43] Li, Y., et al., "A low-cost attitude and heading reference syste, by combination of GPS and Magnetometers and MEMS inertial sensors for mobile applications." Journal of Global Positioning Systems, 2006, Issue 2, Vol. 5, pp. 88-95.

[44] Oh, S.M., Johnson E. N., "Development of UAV Navigations Systems Based on Unscented Kalman Filter." Keystone, CO : s.n., 2006. AIAA Guidance, Navigation and Control Conference and Exhibit. 2006-2621.

[45] Changey, S., Beauvois, D. and Fleck, V., "A Mixed Extended-Unscented Filter for attitude estimation with magnetometer sensor." Minneapolis, MN : s.n., June 14-16, 2006. Proceedings of the 2006 American Control COnference.

[46] Crassidis, J. L. and Lightsey, E. G., "Attitude Determination Using Combined GPS and Three-Axis Magnetometer Data." Breckenridge, CO : s.n., 2000. International Workshop on Aerospace Applications of the Global Positioning System.

[47] Ding, W., Wang, J. and Rizos, C., "Stochastic Modelling Strategies in GPS/INS Data Fusion Process." Surfers Paradise, Australia : s.n., July, 2006. International Global Navigation Satellite Systems Society.

[48] Mohamed, A. H. and Schwarz, K. P., "Adaptive Kalamn Filtering for INS/GPS." Journal of Geodesy, May 1999, Issue 4, Vol. 73, pp. 193-203.

[49] Hu, C., et al., "Adaptive Kalman Filtering for Vehicle Navigation." Journal of Global Poisitioning Systems, November 2003, Issue 1, Vol. 2, pp. 42-47. 
[50] Kim, H., Lee, J. and Park, C., "Performance Improvement of GPS/INS Integrated System Using Allan Variance." Sydney, Australia: s.n., 2004. The 2004 International Symposium of GNSS/GPS.

[51] El-Diasty, M. and Pagiatakis., "Calibration and Stochastic Modelling of Inertial Navigation Sensor Errors." Journal of Global Positioning Systems, s.l. : CPGPS, 2008, Issue 2, Vol. 7, pp. 170-182.

[52] Kim, K. H., Lee, J. G. and Park, C. C., "Adaptive Two-State Extended Kalman Filter for a FaultTolerant INS-GPS Loosely Coupled System." IEEE Transactions on Aerospace and Electronics Systems, s.l. : IEEE, January 2009, Issue 1, Vol. 45. T-AES/45/1/932008..

[53] Fox, J., "Forward and Inverse Stochastic Filtering for Inetial Sensor Calibration." Lanzarote, Spain: International Association of Science and Technology for Development, 2006. Proceedings of the 25th IASTED International Conference on Modeling, Identification and Control. pp. 429-434. 1025-8973.

[54] Kerr, T., "Decentralized Filtering and Redundancy Management for Multisensor Navigation." IEEE Transactions on Aerospace and Electronic Systems, January 1987, Issue 1, Vols. AES-23. 00189251/87/0100-0083.

[55] Fauske, K., Hallingstad, O., Hegrenaes, O., "Toward a Framework for High Integrity Navigation of Autonomouse Underwater Vehicles." Lakeside Hotel, Ireland: s.n., 2008. 2nd Internation Federation of Automatic Control Workshop on Navigation. Vol. 2. 10.3182/20080408-3-IE4914.00033.

[56] Yang, F., Cheng, C., Pan, Q., Zhang, G., "Practical Integrated Navigation Fault Detection Algorithm Based on Sequential Hypothesis Testing." Journal of Systems Engineering and Electronics, February 2011, Issue 1, Vol. 22, pp. 146-149.

[57] Carlson, N., "Federated Square Root Filter for Decentralized Parallel Processes." IEEE Transactions on Aerospace Electronic Systems, May 1990, Issue 3, Vol. 26. 0018-9251/90/05000517.

[58] Carlson, N., "Federated Filter for Fault-Tolerant Integrated Navigation Systems." Orlando, FL : IEEE, 1988. Position Location and Navigation Symposium,. pp. 110-110. 10.1109/PLANS.1988.195473 .

[59] Mirabadi, A., Mort, N., Schmid, F., "Fault Detection and Isolation in Multisensor Train Navigation Systems." 1998. UKACC International Conference on Control. pp. 969-974. 
[60] Bae, J., Yoon, S., Kim, Y., "Fault-Tolerant Attitude Estimation for Satellite using Federated Unscented Kalman Filter." [ed.] J. Hall. Advances in Spacecraft Technologies. s.1. : InTech, 2011, p. Chapter 11. Available from: http://www.intechopen.com/articles/show/title/faulttolerant-attitude-estimation-for-satellite-using-federated-unscented-kalman-filter.

[61] Duan, F., Wang, H., Zhang, L., "Study on Fault-tolerant Filter Algorithm for Integrated Navigation System." Harbin, China : s.n., 2007. Proceedings of the 2007 IEEE International Conference on Mechatronics and Automation. pp. 2419-2423. 1-4244-0828-8/07.

[62] , "A Brief History of Piccolo Development." [Online] [Cited: January 5, 2009.] www.cloudcaptech,com/piccolo/A_breif_history_of_piccolo_development.pdf.

[63] Bailey, R.M., et al., "Experimental Validation: Subscale Aircraft Ground Facilities and Integrated Test Capability." San Francisco, CA : s.n., August, 2005. AIAA Guidance, Navigation and Control Conference and Exhibit. 2005-6433.

[64] Christopersen, H.B., et al., "Small Adaptive Flight Control Systems for UAVs Using FPGA/DSP Technology." Chicago, IL : s.n., September, 2004. Proceedings of the AIAA "Unmanned Unlimited" Technical Conference, Workshop and Exhibit. 2004-6556.

[65] How, J., Teo, J. and Michini, B., "Adaptive Flight Control Experiments using RAVEN." [Online] [Cited: July 2009, 20.] www.acl.mit.edu/papers/HowYWALS08.pdf.

[66] Campa, G., et al., "On-Line Learning Neural Networks for Sensor Validation for the Flight Control System of a B777 Research Aircraft Model." International Journal of Robust Non-Linear Control, 2002, Vol. 12, pp. 987-1007.

[67] Seanor, B., et al., "3 Aircraft Formation Flight Experiment." Ancona, Italy : s.n., June, 2006. Meterranean Control Conference.

[68] Gu, Y., et al., "Autonomous Formation Flight: Hardware Development." Ancona, Italy : s.n., June, 2006. Mediterranean Control Conference.

[69] Gu, Y., et al., "Design and Flight Testing Evaluation of Formation Flight Control Laws." IEEE Transactions on Control Systems Technology, Nov. 2006, pp. 1105-1112.

[70] Perhinschi, M., Burken, J. and Campa, G., "Comparison of Different Neural Augmentations for the Fault Tolerant Control Laws of the WVU YF-22 Model Aircraft." Anacona, Italy : s.n., June, 2006. Mediterranean Control Conference.

[71] Gu, Y., et al., "Integrated Avionics Systems for UAVs." Honolulu, HI : AIAA, Aug. 2008. AIAA Guidance, Navigation and Controls Conference and Exhibit. 
[72] Gross, J., Gu, Y., Seanor, B., Gururajan, S., Napolitano, M., "Advanced Research Intergrated Avionics (ARIA) System for Fault-Tolerant Flight Research." Chicago : AIAA, 2009. AIAA Guidance, Navigation and Controls Conference and Exhibit.

[73] Campa, G., "RTAI-Target HOWTO." Real Time Application Interface. [Online] [Cited: June 15, 2009.] www.rtai.org.

[74] Rosemount Aerospace, Inc, Goodrich Sensor Systems., VG44 Series Miniature Vertical Gyroscopes Specification Sheet. s.1. : Goodrich Sensor Systems, 2002.

[75] Crossbow Technology., "IMU400CC 6DOF Inertial Measurement Unity." Specification Sheet. San Jose : CA. Document Part Number: 6020-0020-01.

[76] —. "VG400CA (DMU-HDX)." Specification Sheet. San Jose, CA : s.n.

[77] Analog Devices., "Triaxial Inertial Sensor with Magnetometer ADIS16400/ADIS16405." Specification Sheet. Norwood, MA : s.n., July 2009.

[78] Novatel., Novatel OEM GPS: Novatel User's Guide. 2002.

[79] Hao, Y., Xiong, Z., Sun, F., Wang, X., "Comparison of Unscented Kalman Filters." Harbin, China : s.n., August, 2007. Proceedings of the 2007 IEEE International Conference on Mechatronics and Automation. pp. 895-899.

[80] Briers, M. and Maskell, S. R., Wright, R., "A Rao-Blackwellised Unscented Kalman Filter." s.l. : IEEE, 2003. Proceedings of the 6th International Conference of Information Fusion. pp. 55-61. 0-9721844-4-9.

[81] Roskam, J., Airplane Flight Dynamics and Automatic Flight Controls. Lawrence: DAQcorporation, 2003.

[82] Stevens, B. L. and Lewis, F. L., Aicraft Control and Simulation. 2nd Edition. s.1. : Wiley \& Sons, 2003.

[83] Luczak, S., Oleksiuk, W. and Bodnicki, M., "Sensing Tilt With MEMS Accelerometers." IEEE Sensors, December 2006, Issue 6, pp. 1669-1673.

[84] Jarrell, J., Employ Sensor Fusion Techniques for Determining Attitude and Position Information. Department of Mechanical and Aerospace Engineering, West Virginia University. 2008. Master's Thesis.

[85] Jarrell, J., et al., "Aircraft Attitude, Position, and Velocity Determiniation Using Sensor Fusion." Honolulu, HI : AIAA, Aug. 2008. AIAA Guidance, Navigation and Controls Conference and Exhibit. 
[86] Kingston, D. B. and Beard, R. W., "Real-Time Attitude Position Estimation for Small UAVs Using Low-Cost Sensors." Chicago : s.n., 2004. AIAA 3rd Unmanned Unlimited Systems Conference and Workshop. AIAA-2004-6488.

[87] Gross, J., Gu, Y., Rhudy, M., Gururajan, S., Napolitano, M., "Flight-Test Evaluation of Sensor Fusion Algorithms for Attitude Estimation." Submitted to IEEE Transactions on Aerospace Electronic Systems, Jan. 2011.

[88] National Oceanic and Atmospheric Administration., NOAA's Geophysical Data. Geomagnetic Online Calculator. [Online] http://www.ngdc.noaa.gov/IGRFWMM.jsp.

[89] Gross, J., Gu, Y. and Napolitano, M., "A Systematic Approach for Extended Kalman Filter Tuning and Low-Cost Inertial Sensor Calibration within a GPS/INS Application." Toronto, ON, CA : AIAA, 2010. AIAA Guidance, Navigation and Control Conference and Exhibit.

[90] Ding, W., Wang, J. and Rizos, C., "Improving Covariance Based Adaptive Estimation for GPS/INS Integration." Jeju, Korea : s.n., 2006. IAIN/GNSS 2--6.

[91] Allan, D. W., "Statistics of atomic frequency standards." Proceedings IEEE, s.l. : IEEE, February 1966, Issue 2, Vol. 54.

[92] Xing, Z., Over-bounding Integrated INS/GNSS Output Errors. The University of Minnesota. Minneapolis : s.n., October, 2010. PhD Thesis.

[93] Zhang, X., Li, Y. and Mumford, P., Rizos, C., "Allan Variance Analysis on Error Characters of MEMS Inertial Sensors for an FPGA-based GPS/INS System." Yokohama, Japan : s.n., 2008. International Symposium on GPS/GNSS. pp. 127-133.

[94] Yi, Y., On Improving the Accuracy and Reliability of GPS/INS-Based Direct Sensor Georeferencing. Geodetic Science and Surveying Graduate Program, The Ohio State Univesity. Columbus, $\mathrm{OH}$ : s.n., 2007. PhD Thesis.

[95] Vasconcelo, J. F., Elkain, G., Silvestre, C., Oliveria, P., Cardeira, B., "A geometric approach to strapdown magnetometer calibration in sensor frame." Killaloe, Ireland : s.n., 2008. Proceedings of IFAC Workshop on Navigation, Guidance and Control of Underwater Vehicles.

[96] Gross, J., et al., "A Comparison of Extended Kalman FIlter, Sigma-Point Kalman Filter, and Particle Filter in GPS/INS Sensor Fusion." Toronto, ON, Canada: AIAA, 2010. AIAA Guidance, Navigation and Control COnference and Exhibit . 
[97] Ruhdy, M., Gu, Y., Gross, J., Napolitano, M., "Sensitivity Analysis of EKF and UKF in GPS/INS Sensor Fusion." Portland : s.n., 2011. AIAA Guidance Navigation and Control Conference and Exhibit.

[98] Gu, Y., Gross, J., Rhudy, B., Gururajan, S., Napolitano, M., "A Fault-Tolerant Multiple Sensor Fusion Approach Applied to UAV Attitude Estimation." Submitted to IEEE Transactions on Robotics and Automation, February 2011.

[99] Gross, J., Gu, Y., Rhudy, M., Barchesy, F., Napolitano, M., "On-Line Modeling and Calibration of Low-Cost Navigation Sensors." Portland, OR : s.n., 2011. Abstract Accepted 2011 AIAA Modeling and Simulation Technologies Conference.

[100] Mitchell, T. M., Machine Learning. s.1. : McGraw-Hill Companies, Inc., 1997. 0-070-042807-7.

[101] Bumback, B. and Srinath, M., "A Chi-Square Test for Fault-Detection in Kalman Filters." IEEE Transactions on Automatic Control, June 1987, Issue 6, Vols. AC-32. 0018-9286/87/0600-0552.

[102] IEEE., IEEE Standard Format Guide and Test Procedure for Single-Axis Interometric FIber Optic Gyros. IEEE Std. 952-1997.

[103] Xing, Z.1 Gebre-Egziabher, D., "Modeling and Bounding Low Cost Inertial Sensor Errors." Monterey, CA : IEEE, 2008. IEEE/ION Position, Location and Navigation Symposium. pp. 1122 - 1132 . 978-1-4244-1536-6. 


\section{Appendix A. Discretized Models and Jacobian Matrices}

In order to implement the GPS/INS sensor fusion algorithm using a nonlinear filter, the continuous time formulations must be discretized. This was done using a first order Euler approximation. For the case of the EKF, Jacobian matrices that are comprised of first order Taylor series expansion are included. This Appendix includes the discretized models of the various GPS/INS sensor fusion formulations.

\section{A.1 9-State Formulation}

The discrete equivalent of the 9-state formulation is provided in A.1.

$$
\left[\begin{array}{c}
x_{k} \\
y_{k} \\
z_{k} \\
V_{x_{k}} \\
V_{y_{k}} \\
V_{z_{k}} \\
\phi_{k} \\
\theta_{k} \\
\psi_{k}
\end{array}\right]=\left[\begin{array}{c}
x_{k-1}+T s V_{x k-1} \\
y_{k-1}+T s V_{y_{k-1}} \\
z_{k-1}+T s V_{z k-1} \\
V_{x_{k-1}}+T s\left(\cos \psi_{k-1} \cos \theta_{k-1} a_{x_{k-1}}+\left(-\sin \psi_{k-1} \cos \phi_{k-1}+\cos \psi_{k-1} \sin \theta_{k-1} \sin \phi_{k-1}\right) a_{y_{k-1}}+\left(\sin \psi_{k-1} \sin \phi_{k-1}+\cos \psi_{k-1} \sin \theta_{k-1} \cos \phi_{k-1}\right) a_{z_{k-1}}\right) \\
V_{y_{k-1}}+T s\left(\sin \psi_{k-1} \cos \theta a_{x_{k-1}}+\left(\cos \psi_{k-1} \cos \phi_{k-1}+\sin \psi_{k-1} \sin \theta_{k-1} \sin \phi_{k-1}\right) a_{y_{k-1}}+\left(-\cos \psi_{k-1} \sin \phi_{k-1}+\sin \psi_{k-1} \sin \theta_{k-1} \cos \phi_{k-1}\right) a_{z_{k-1}}\right) \\
V_{z_{k-1}}+T s\left(-\sin \theta_{k-1} a_{x k-1}+\cos \theta_{k-1} \sin \phi_{k-1} a_{y_{k-1}}+\cos \theta_{k-1} \cos \phi_{k-1} a_{z k-1}-g\right) \\
\phi_{k-1}+T s\left(p_{k-1}+q_{k-1} \sin \phi_{k-1} \tan \theta_{k-1}+r_{k-1} \cos \phi_{k-1} \tan \theta_{k-1}\right) \\
\theta_{k-1}+T s\left(q_{k-1} \cos \phi_{k-1}-r_{k-1} \sin \phi_{k-1}\right) \\
\psi_{k-1}+T s\left(q_{k-1} \sin \phi_{k-1}+r_{k-1} \cos \phi_{k-1}\right) \sec \theta_{k-1}
\end{array}\right]
$$

Since the Kalman Filter equations only apply to a linear model, the system equations (A.1) are linearized by using a first order Taylor series expansion leading to the generation of a $9 x 9$ 
Jacobian matrix. The linearized state relationship matrix is indicated as $\boldsymbol{A}$ within Eq. A.3, and is shown in expanded form

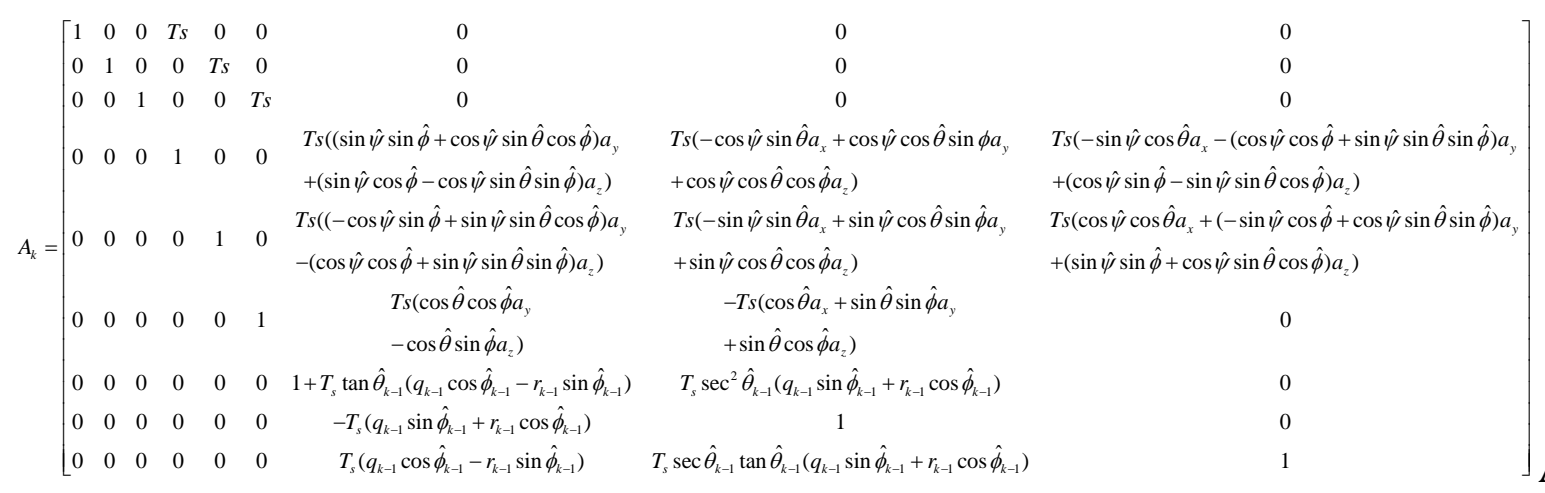

\section{A.2 3-State Formulation}

The 3-state formulation separates the IMU rate gyros and accelerometers between the prediction and measurement procedures, respectively; however the same relationships are present. Therefore, the linear $\boldsymbol{A}$ matrix consists of indices (7:9x7:9) of (A.2), and the linear $\boldsymbol{H}$ matrix consists of indices $(4: 6 x 4: 6)$.

\section{A.3 15-State Formulation}


The 15-state formulation augments the IMU input with six estimated bias parameters. Therefore upper left-hand $9 x 9$ sub-diagonal block of $\boldsymbol{A}$ is the same as the 9 -state formulation (0.3), however the IMU measurements have the bias estimates negated from then. Furthermore, additional terms in the Jacobian arise due to partial derivatives with respect to the bias parameters. The first $3 \times 3$ block arises by taking partials of the velocity update equations $(4: 6) x(10: 12)$

$$
J 1=\left[\begin{array}{ccc}
-\cos \psi \cos \theta & \sin \psi \cos \phi-\cos \psi \sin \theta \sin \phi & -\sin \psi \sin \phi-\cos \psi \sin \theta \cos \phi \\
-\sin \psi \cos \theta & -\cos \psi \cos \phi-\sin \psi \sin \theta \sin \phi & \cos \psi \sin \phi-\sin \psi \sin \theta \\
0 & -\sin \phi \tan \theta & -\cos \phi \tan \theta
\end{array}\right] T s
$$

The second $3 \times 3$ block is calculated by taking partials of the velocity update equations $(7: 9) x(13: 15)$

$$
J 2=\left[\begin{array}{ccc}
-1 & -\sin \phi \tan \theta & -\cos \phi \tan \theta \\
0 & -\cos \phi & \sin \phi \\
0 & -\sin \phi \sec \theta & -\cos \phi \sec \theta
\end{array}\right] T s
$$

Therefore, the resulting linear $A$ matrix for the 15 -state formulation is given: 


$$
A_{15 \times 15}=\left[\begin{array}{ccc} 
& \multicolumn{2}{c}{0_{6 \times 3}} \\
A_{9 x 9} & J_{1} & 0_{3 \times 3} \\
& 0_{3 \times 3} & J_{2} \\
0_{6 \times 6} & I_{6 \times 6}
\end{array}\right]
$$

Finally, the linear observation function used within the 15-state formulation is simulate to the 9state, but is horizontally concatenated with a $6 x 6$ matrix of zeros.

\section{A.4 Non-Additive Noise}

When considering non-additive noise, the assumption used in this study was the noises were additive at the sensor level. Therefore additional Jacobians of the state equations with respect to additive noise on the inputs were used. Because the functions are linear with respect to the input vector, these Jacobians were simply the state transition functions. For the 9-state formulation, the derivative of $f$ with respect to the acceleration inputs is the DCM representing the velocity update equations, and additive noise on the rate gyros were propagated using the attitude kinematic equations. 


\section{Appendix B. Joint-Estimation vs. Dual-Estimation for On-Line IMU bias tracking}

As indicated in Chapter 6 originally both dual and joint state and parameter estimation approaches were considered. However, a dual state and parameter filter assumes that the offdiagonal terms of the error-covariance matrix between the states and parameters are zero (Equation( 6.1)). In this appendix, the poor performance of the dual state approach is shown in comparison to the joint approach. Also, the cross-covariance terms of the joint filter between the states and IMU biases are shown to have a magnitude that is significant in comparison to the diagonal terms. Figure B.1 shows the comparison of estimation performance over 23 flights.

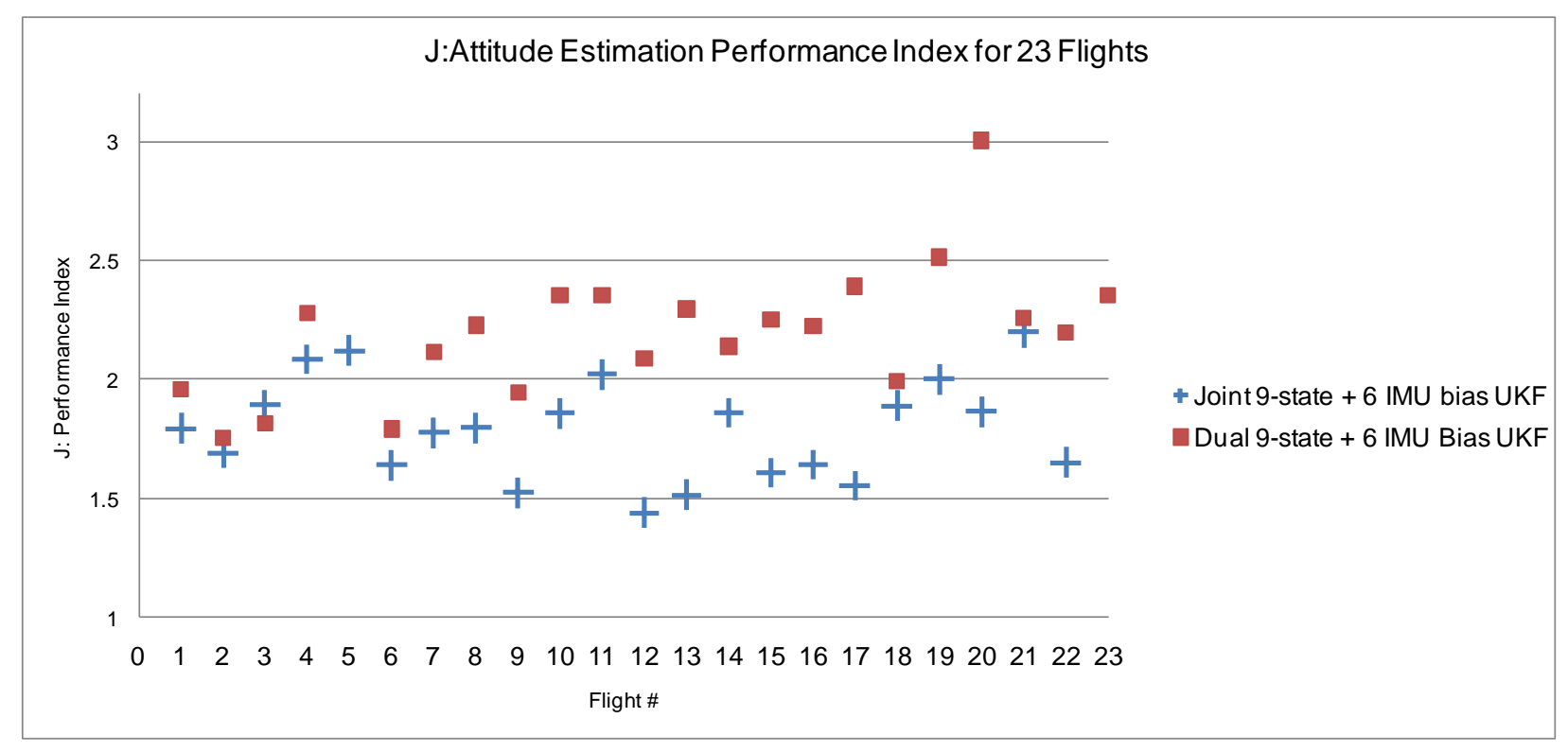

Figure B.1: Performance comparison of Joint vs. Dual Bias Estimation

The estimation performance of the dual state and bias tracking filter is significantly worse than the joint approach (average $\mathrm{J}=2.244$ vs. 1.777 ). In order to illustrate that the cross terms on the 
parameters with respect to the states are significant, Figure B.2 shows a time history of the magnitudes of some block diagonal sections of $\boldsymbol{P} \boldsymbol{x}$.

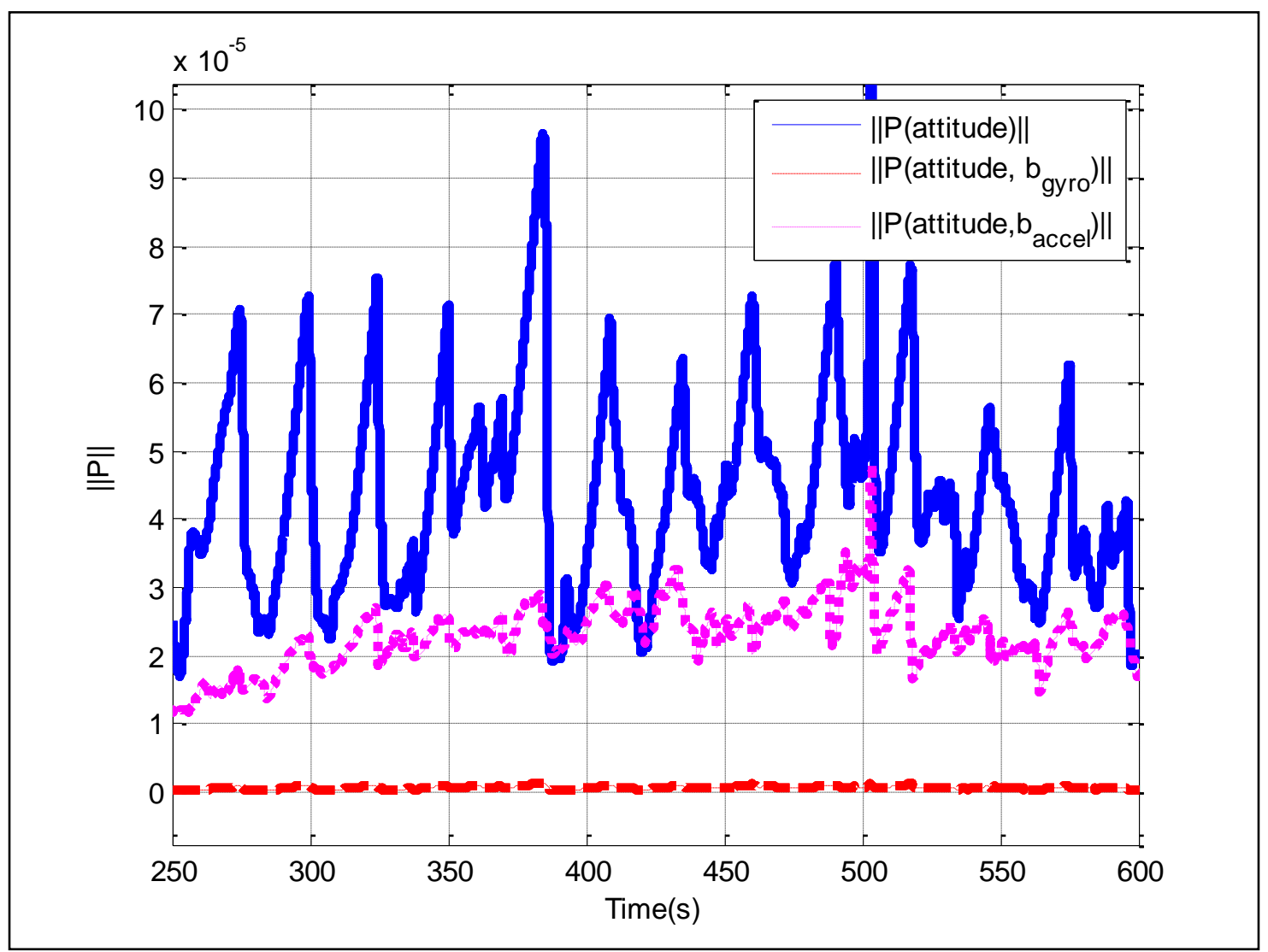

Figure B.2: Time history of cross-covariance terms of states and parameters in joint state and parameter estimator

Figure B.2 shows that the cross-covariance of the attitude with respect to the accelerometer biases has a significant magnitude and therefore would greatly contribute to the calculation of the Kalman gain. 